\title{
A catalogue of low-mass X-ray binaries ${ }^{\star}$
}

\author{
Q. Z. Liu ${ }^{1,2}$, J. van Paradijs², and E. P. J. van den Heuvel $^{2}$ \\ 1 Purple Mountain Observatory National Astronomical Observatories, Chinese Academy of Sciences, \\ Nanjing 210008, PR China \\ 2 Astronomical Institute "Anton Pannekoek", University of Amsterdam, Kruislaan 403, 1098 SJ Amsterdam, \\ The Netherlands
}

Received 30 October 2000 / Accepted 11 January 2001

\begin{abstract}
We present a catalogue of low-mass X-ray binaries. The catalogue is an updated version of the catalogue of van Paradijs (1995). This new catalogue contains 150 sources, 31 new low-mass X-ray binaries in addition to the 119 sources listed in van Paradijs' catalogue. The aim of this catalogue is to help the reader gain easy access to the recent literature (up to about August 2000) on individual sources, and to provide of some basic information on the X-ray sources and their counterparts in other wavelength ranges (UV, optical, IR, and radio). In cases where there is some doubt about the low-mass nature of the X-ray binary, this is mentioned. In an appendix we list the Anomalous X-ray Pulsars (AXPs), which nowadays are no longer thought to be low-mass X-ray binaries.
\end{abstract}

Key words. X-ray: stars - (stars:) binaries: general - catalogs - pulsars: general

\section{Introduction}

An X-ray binary contains either a neutron star (NS) or a black hole $(\mathrm{BH})$ accreting material from a companion star. Thus, cataclysmic variables (CVs) are not included among the X-ray binaries. X-ray binaries can be further divided into two different classes according to the mass of the companion star: high-mass X-ray binaries (HMXB) and low-mass X-ray binaries (LMXB). In a previous paper we presented an up-to-date catalogue of high-mass X-ray binaries (Liu et al. 2000).

The secondary of LMXB systems is a low-mass (in general $M \leq 1 M_{\odot}$ ) star, which transfers matter by Rochelobe overflow. Among the low-mass companion stars we find white dwarfs, late-type main-sequence stars, two A-type stars and F-G-type sub-giants. The last-mentioned category of companion stars may well be the mass-transfer remnants of stars that originally were of intermediate mass $\left(M \sim 1.5\right.$ to $\left.4 M_{\odot}\right)$ as has recently been suggested for Cygnus X-2 (cf. Podsiadlowski \& Rappaport 2000; Tauris et al. 2000). The optical counterparts of LMXBs are intrinsically faint objects. The spectra of most of them show a few characteristic emission lines superposed on a rather

Send offprint requests to: Q. Z. Liu,

e-mail: qzliu@mail.pmo.ac.cn

* Table 1 is also available in electronically at the CDS via anonymous ftp $(130.79 .128 .5)$ or via

http://cdsweb.u-strasbg.fr/cgi-bin/qcat?J/A+A/368/1021 flat continuum. The optical continuum of LMXBs is dominated by the emission from an accretion disk around the compact star, which is predominantly the result of reprocessing of a fraction of the X-rays into optical photons in the disk. The contribution from the secondary is generally negligible. On occasion, however, the presence of the secondary can be discerned in the spectrum (or colors) of the LMXBs. This particularly is the case for systems with donors that are or started out as intermediate mass stars, such as Her X-1 and Cyg X-2. For a full understanding of LMXBs in various aspects one can refer to the book by Lewin et al. (1995).

The classification as LMXB is mainly based on the spectra obtained from an optical identification, and/ or on the mass function from X-ray pulse arrival time measurements. If neither is available, a classification may be inferred based on the similarity of the X-ray properties to other identified systems. An unidentified system is classified as an LMXB containing a neutron star if one or more of the following properties are observed:

- type I X-ray bursts (which to date have only been seen from neutron stars in LMXBs);

- the $1-10 \mathrm{keV}$ spectrum is soft with a characteristic temperature of 5-10 keV and/or;

- the orbital period is less than about $12 \mathrm{hr}$. 
In 1983 the number of known LMXBs was about 33 (van Paradijs 1983; Bradt \& McClintock 1983). By the time of the previous catalogue the number of X-ray sources associated with low-mass stars had increased to 119 (van Paradijs 1995) (with three supersoft X-ray sources (SSSs) and two AXPs excluded (see Sect. 3)). In this paper we present a new catalogue, which includes the information extracted from the literature up to August 2000 and increases the number of known LMXBs to 150, including 31 newly discovered LMXBs as well as the 119 "old" ones listed in van Paradijs' catalogue.

\section{Description of the table}

Table 1 lists the 150 LMXBs. The format of the table is similar to that of the previous one (van Paradijs 1995) and the well-known work of Bradt \& McClintock (1983), of which the present catalogue is meant to be an update. In the table the sources are ordered according to right ascension; part of the (mainly numerical) information on a source is arranged in seven columns, below which for each source additional information is provided in the form of key words with reference numbers [in square brackets]. When a result is unreliable, a colon (:) or a question mark (?) will follow the adopted entry. The columns have been arranged as follows:

In Col. 1 the first line contains the source name, with rough information on its sky location according to the convention $h h m m \pm d d d$. Here hh and $\mathrm{mm}$ indicate the hours and minutes of right ascension, ddd the declination in units of 0.1 degree (in a small number of cases, the coordinates shown in the name are given with more, or fewer, digits). However, for a ROSAT source the name is always given in the form of hhmm.m \pm ddmm. The prefix $\mathrm{J}$ indicates a name based on J2000 coordinates. Otherwise, 1950 coordinates were used in the name. Alternative source names are given in the second line. The third line of Col. 1 lists survey catalogues and experiments in which the source was listed and detected, respectively. The following abbreviations have been used.

- A: Ariel V sky survey;

- AS: ASCA;

- B: BeppoSAX;

- C: Compton $\gamma$-ray Observatory;

- E: Einstein Observatory;

- Exo: Exosat;

- G: Ginga;

- Gr: Granat;

- H: HEAO A-1 sky survey;

- Ha: Hakucho;

- I: Indian X-ray Astronomy Experiment (IXAE);

- K: Kvant;

- M: MIT OSO-7 sky survey;

- OAO: Orbiting Astronomical Observatory;

- R: ROSAT;

- S: SAS 3;

- SL: Space Lab;
- T: Tenma;

- U: Uhuru sky survey;

- V: Vela-5 and -6 satellites;

- X: Rossi XTE.

In the first line of Col. 2, the source types are indicated with a letter code, as follows:

- A: atoll source (18);

- B: X-ray burst source (63);

- D: "dipping" low-mass X-ray binary (11);

- G: globular-cluster X-ray source (13);

- P: X-ray pulsar (5);

- T: transient X-ray source (76);

- U: ultra-soft X-ray spectrum. These sources include black-hole candidates; some "extreme ultra-soft" (EUS) sources may be white dwarfs on whose surface steady nuclear burning takes place.

- Z: Z-type (7).

In the third line of Col. 2, we provide some information on the type of observation from which the source position has been derived. The following abbreviations have been used: o, optical; x, X-ray; r, radio; IR, infrared. A reference on the source position is given below the columnar information under "Pos.". In addition, we give an indication of the accuracy of this position, in the form of equivalent (90 percent confidence level) error radii, but in several cases this can only be considered an approximation (e.g. when the error box is not circular). When no accuracy is quoted, it is about one arcsecond or better.

Column 3 contains in the first two lines the right ascension (RA) and declination (DEC) of the source for epoch 1950 for the usual name, and for epoch 2000 for the sources with the names of J2000 coordinates. RA is given as hhmmss.s to an accurcy of $0.1 \mathrm{~s}$, DEC is given in ${ }^{\circ} \prime \prime \prime$, to an accuracy of $1^{\prime \prime}$. The third line gives the galactic longitude and latitude to an accuracy of $0.1^{\circ}$ (except for sources close to the galactic center (GC), where these coordinates are given to $0.01^{\circ}$ ).

The first and second lines of Col. 4 give names of an optical counterpart. The third line contains a reference to a finding chart. An asterisk followed by a number or letter refers to star numbers used in the finding chart; "star" refers to a star in the finding chart that has not been assigned a number or letter. Many optical counterparts have been indicated with a variable-star name, as given in the General Catalogue of Variable Stars and in recent name lists of variable stars as published regularly in the IAU Information Bulletin on Variable Stars, or a number in a well-known catalogue (e.g. HD, SAO). For X-ray sources in globular clusters, the cluster name is here given, in addition to the name of a stellar optical counterpart.

The fifth column contains some photometric information on the optical counterpart. In the first line, the apparent visual magnitude, $V$, and the color indices $B-V$, and $U-B$, are listed. The second line contains the estimate of the interstellar reddening, $E_{B-V}$. 
Table 1. Low-mass X-ray binaries

\begin{tabular}{|c|c|c|c|c|c|c|}
\hline $\begin{array}{l}\text { Source } \\
\text { Name(s) } \\
\text { Spacecraft }\end{array}$ & $\begin{array}{l}\text { type } \\
\text { Pos. }\end{array}$ & $\begin{array}{l}\mathrm{RA} \\
\mathrm{DEC} \\
l^{\mathrm{II}}, b^{\mathrm{II}}\end{array}$ & $\begin{array}{l}\text { Opt. Ctp. } \\
{[\mathrm{FC}]}\end{array}$ & $\begin{array}{l}V, B-V, U-B \\
E_{B-V}\end{array}$ & $\begin{array}{l}F_{\mathrm{x}} \\
\mu \mathrm{Jy}\end{array}$ & $\begin{array}{l}P_{\text {orb }}(\mathrm{hr}) \\
P_{\text {pulse }}(\mathrm{s})\end{array}$ \\
\hline $\begin{array}{l}\text { 0042+323 } \\
\text { XN Per } 1992 \\
\mathrm{U}, \mathrm{M}, \mathrm{A}, \mathrm{AS}, \mathrm{R}, \mathrm{B}, \mathrm{X}\end{array}$ & $\begin{array}{l}\mathrm{T} \\
\times 60^{\prime \prime}\end{array}$ & $\begin{array}{l}004208.5 \\
+324453 \\
121.3,-29.8\end{array}$ & $\begin{array}{l}{ }^{*} 3 \\
{[168]}\end{array}$ & $\begin{array}{l}19.3,0.6,-- \\
0.2 \\
{[168]}\end{array}$ & $\begin{array}{l}<0.5-55 \\
{[103]}\end{array}$ & \\
\hline \multicolumn{7}{|c|}{ Pos.: [1070]; transient: [726, 1070, 1402]; opt. cpt. uncertain: [74]. } \\
\hline $\begin{array}{l}\mathrm{J} 0422+32 \\
\mathrm{C}, \mathrm{Gr}, \mathrm{R}, \mathrm{K}, \mathrm{AS}, \mathrm{X}\end{array}$ & $\begin{array}{l}\mathrm{T} \\
\mathrm{o}, \mathrm{r}\end{array}$ & $\begin{array}{l}041829.9 \\
+324724 \\
197.3,-11.9\end{array}$ & & $\begin{array}{l}13.2,0.3,-0.5 \\
0.23-0.4 \\
{[1151,1158,1159]}\end{array}$ & $\begin{array}{l}3000 \\
(20-300 \mathrm{keV}) \\
{[433,991]}\end{array}$ & $\begin{array}{l}5.1 \\
{[125,150,664,980]}\end{array}$ \\
\hline
\end{tabular}

Pos.: [871]; outburst: [156, 991, 1160]; hard X-rays: [131, 1341]; QPO: [692, 1325]; X-ray phase lag: [479, 1325]; BHC: [157, 1042, 1243]; opt. cpt.: [157]; opt. sp.: [88, 151, 408, 1391]; opt. phm.: [126, 193, 675]; opt. sp. and phm.: [54, 159, 835, 980]; $10 \mu \mathrm{m}$ emission: [1357]; UV/opt./radio obs.: [1159]; UV/opt. sp.: [591]; opt. sp. and phm./X ray: [125]; 16.18 hr period?: [835]; UV sp.: [1158]; orbital parameters: [675, 980]; M2V star: [365]; BHC mass: [66, 126, 151, 365, 408]; distance: [126, 1159]; IR lt. curve: [66]; radio obs.: [493].

\begin{tabular}{llllll}
\hline $0512-401$ & GB & 051227.9 & NGC 1851 & & $3-6$ \\
$\mathrm{U}, \mathrm{M}, \mathrm{A}, \mathrm{H}, \mathrm{G}$, Exo & $\mathrm{x} 2^{\prime \prime}$ & $244.5,-35.0$ & {$[475]$} & {$[2]$} & {$[124,999,1366]$}
\end{tabular}

Pos.: [475, 525]; opt. studies glob. cl.: [2, 29, 278, 528, 1109]; cluster center: [525, 1154]; cluster moderately metal poor: [10]; $\mathrm{X}$ rays: $[124,345]$; X-ray bursts: [216, 225, 382]; radio obs.: [785].

\begin{tabular}{lllllll}
\hline $0521-720$ & Z? & 052118.0 & ${ }^{*} 22$ & $18 .-19 ., 0.0,-0.8$ & $9-44$ & 8.16 \\
LMC X-2 & & -720026 & & 0.1 & & \\
U, M, A, H, X, R & о $3^{\prime \prime}$ & $283.1,-32.7$ & {$[637]$} & {$[121,260,778]$} & {$[103]$} & {$[121,1173]$}
\end{tabular}

Pos.: [103]; X-ray obs.: [1231]; Z source?: [1130, 1173]; opt. id.: [774, 992, 993]; 12.5 d orb. period(?): [260]; coordinated X-ray/opt. obs.: [89]; opt. spectrum: [89, 923]; near-IR spectrum: [255].

\begin{tabular}{|c|c|c|}
\hline J0532.7-6926 & $\begin{array}{l}053242.8 \\
-692618\end{array}$ & 0.5 \\
\hline $\mathrm{R}$ & $279.9,-32.1$ & {$[484]$} \\
\hline
\end{tabular}

Pos.: [1119]; in LMC: [484]; black hole candidate (?): [484].

\begin{tabular}{llllll}
\hline $0614+091$ & AB & 061422.8 & V1055 Ori & $18.5,0.3,-0.5$ & 50 \\
U, M, A, H, & & +090922 & & 0.3 & \\
S, Gr, E, Exo, X, B & o & $200.9,-3.4$ & {$[323,447]$} & {$[287]$} & {$[1399,1448]$}
\end{tabular}

Pos.: [103]; X-ray obs.: [42, 1043]; X-ray bursts: [108, 109, 782, 1250]; atoll source: [876, 1163]; kHz QPOs: [374]; kHZ QPO study: [378, 1364]; millisecond pulsation: [374]; hard/soft X-ray anticorrelated: [377]; hard X-ray lag: [375]; opt. cpt.: [287, 939]; optical spectrum: [786]; coordinated X-ray/opt. obs.: [786]; long-term variability: [786, 830]; radio obs.: [333]; distance: [109].

\begin{tabular}{lllllll}
\hline $0620-003$ & TU & 062011.1 & V616 Mon & $11.2,0.2,-0.8$ & $<0.02-50000$ & 7.75 \\
N Mon 1975 & & -001911 & & 0.4 & {$[774,1407]$} & {$[857]$}
\end{tabular}

Pos.: [103]; bright transient 1975: [343, 1407]; previous outburst 1917: [336]; hard X rays: [233]; optical outburst: [967, 1407]; 7.8 day modulation in outburst: [332, 771, 849, 1057, 1095, 1300, 1401]; quiescent X rays: [300, 774]; quiescent opt. spectra (K5) \& mass function (BH): [512, 635, 820, 857, 858, 941]; UV obs.: [859, 869, 1452]; near-IR sp.: [255, 1149]; near IR phm. obs.: [1138]; long-term lt. curve: [737]; quiescent opt. phm., $V_{\text {quiesc }}=18.2$ : [857, 858]; orbital light curves: [513]; opt. polarimetry: [316]; radio obs.: [293, 333, 416, 989]; multiple radio jet ejection: [718]; distance: [1138]; model: [347].

\begin{tabular}{llll}
\hline $0656-072$ & $\mathrm{~T}$ & 065601 & $20-80$ \\
$\mathrm{~A}$ & $\mathrm{x} 3^{\prime}$ & $220.2,-1.7$ & {$[143,215,656]$}
\end{tabular}

Pos.: [143]; relatively soft X-ray spectrum: [230]. 
Table 1. continued

\begin{tabular}{lllllll}
\hline Source & type & RA & Opt. Ctp. & $V, B-V, U-B$ & $\begin{array}{l}F_{\mathrm{x}} \\
\mu \mathrm{Jy}\end{array}$ & $\begin{array}{l}P_{\text {orb }}(\mathrm{hr}) \\
P_{\text {pulse }}(\mathrm{s})\end{array}$ \\
Name(s) & & DEC & & & \\
Spacecraft & Pos. & $l^{\mathrm{II}}, b^{\mathrm{II}}$ & {$[\mathrm{FC}]$} & & \\
\hline $0748-676$ & TBD & 074825.0 & UY Vol & $16.9-17.5,0.1,-0.9$ & $0.1-60$ & 3.82 \\
& & -673732 & & 0.42 & {$[443]$} & {$[998,1001]$}
\end{tabular}

Pos.: [1262]; orbital decay: [12, 533, 1001, 1180]; X-ray obs.: [212, 401]; X-ray dips: [998, 1180, 1181]; X-ray spectrum: [1420]; X-ray bursts: [282, 443, 444]; soft X-ray excess: [1279]; QPOs: [573, 577]; X-ray/opt. obs.: [1278]; optical spectra \& photometry: [253, 259, 929, 1125, 1352]; near-IR sp.: [255]; a triple system(?): [239]; not a triple sys.: [532]; $V_{\text {quiesc }}>23$ : [1262].

\begin{tabular}{llll}
\hline J0835.9+5118 & TB & 083556 & $<6$ \\
B & & +5118.6 & {$[399]$}
\end{tabular}

Pos.: [399]; type-I X-ray burst (?): [399]; peak flux of burst of 1 Crab (2-25 keV): [399].

\begin{tabular}{|c|c|c|c|}
\hline $0836-429$ & $\mathrm{~TB}$ & $\begin{array}{l}083537 \\
-4242.6\end{array}$ & $1-55$ \\
\hline $\mathrm{U}, \mathrm{M}, \mathrm{Gr}, \mathrm{G}, \mathrm{R}$ & $\mathrm{x} 1^{\prime}$ & $261.9,-1.1 \quad[72]$ & {$[230,511,794]$} \\
\hline
\end{tabular}

Pos.: [511, 1239]; 10" error J2000 pos. (08 37 23.6, -42 54 02): [72]; northern of two X-ray sources: [450, 795]; X-ray bursts: [7, 794, 795]; X-ray/opt. obs.: [72]; likely MX 0836-42: [230, 813].

\begin{tabular}{|c|c|c|c|c|c|}
\hline $0918-549$ & -1 & $\begin{array}{l}091854.7 \\
-545937\end{array}$ & ${ }^{*} \mathrm{X}$ & $\begin{array}{l}21.0,0.3,-0.9 \\
0.3\end{array}$ & 10 \\
\hline
\end{tabular}

Pos.: [187]; optically underluminous (?): [187]; X-ray line emission: [1130].

\begin{tabular}{lllllll}
\hline $0921-630$ & $\mathrm{D}$ & 092125.1 & V395 Car & $15.3,0.6,-0.5$ & 3 & 216.2 \\
& & -630448 & & 0.2 & & \\
A, S, Exo & o & $281.8,-9.3$ & {$[760]$} & {$[113,186,847,1348]$} & {$[760,847]$} & {$[846,847]$}
\end{tabular}

Pos.: [103]; partial X-ray eclipse (ADC source): [847]; high-resol. sp., secondary star K0 III: [1148]; halo object: [251]; opt. obs.: [185,186]; opt./X-ray obs.: [113]; near-IR sp.: [255]; radio: [1471]; orbital period 13.25 d (?): [703].

\begin{tabular}{|c|c|c|c|c|c|}
\hline $\begin{array}{l}1009-45 \\
\text { XN Vel } 1993 \\
\text { C, AS, Gr }\end{array}$ & $\begin{array}{l}\mathrm{TU} \\
\mathrm{o}\end{array}$ & $\begin{array}{l}101132 \\
-444941 \\
275.4,+9.4\end{array}$ & $\begin{array}{l}{ }^{*} 1 \\
{[306,1140]}\end{array}$ & $\begin{array}{l}14.71,0.13,-- \\
0.20 \\
{[306]}\end{array}$ & $\begin{array}{l}800 \\
(1-10 \mathrm{keV}) \\
{[1263]}\end{array}$ \\
\hline
\end{tabular}

Pos.: [302]; BHC: [430, 704, 1140]; hard X-ray obs.: [430, 704]; distance 1.5-4.5 kpc: [306, 1361]; orbital period 4 hr, or $4.79 \mathrm{hr}$, or $6.86 \mathrm{hr}$ : [306, 839, 1140]; H $\alpha$ emission: [306, 1140]; binary inclination: [1140]; opt. obs.: [306, 839, 1140]; opt. outburst: [28]; opt. reflare: [839]; sp. type later than G5: [306, 1140].

\begin{tabular}{lllllll}
\hline J1118+480 & TA? & 111810.85 & ${ }^{*} 1$ & $12.25-18.8,--,--$ & 40 & 4.1 \\
AS, X, C & o $0.2^{\prime \prime}$ & $157.6,+62.3$ & {$[1312]$} & $<0.024$ & $(2-12 \mathrm{keV})$ & {$[232,1312]$}
\end{tabular}

Pos.: [1312]; transient: [1084]; first BHC in galactic halo (?): [1090, 1312]; IR obs.: [177]; inclination angle: [232, 410, 598, 1312]; low X-ray to opt. flux ratio: [232, 1312]; QPOs: [515, 1090, 1454]; distance and sp. type: [1312]; UV var. lags behind X-rays by 1-2 s, and characteristic of an atoll-type source: [515]; radio cpt.: [1049]; radio obs.: [314]; multiwvl. obs. during low-hard state: [598]; EUVE obs.: [854]; UV obs.: [514]; opt. obs.: [232, 1007, 1312, 1451]; sp. typical of an X-ray nova in outburst: [410].

\begin{tabular}{|c|c|c|c|c|c|}
\hline $\begin{array}{l}\text { 1124-684 } \\
\text { N Mus } 1991\end{array}$ & $\mathrm{TU}$ & $\begin{array}{l}112418.5 \\
-682402\end{array}$ & $\begin{array}{l}13.6,0.3,-- \\
0.25\end{array}$ & $<4-3000$ & 10.4 \\
\hline $\mathrm{Gr}, \mathrm{G}, \mathrm{C}$ & o & $295.0,-6.1 \quad[303]$ & {$[303,435]$} & {$[1235,1241]$} & {$[27,867,1075]$} \\
\hline
\end{tabular}

Pos.: [303]; bright X-ray transient Jan. 1991: [107, 452, 680, 781, 1235]; hard X-ray sp., strong soft component: [337, 1235]; annihilation line near $500 \mathrm{keV}$ : [422, 432, 1242]; QPO: [75, 1259]; similar to 0620-000: [304]; opt. outburst: [27]; opt. sp. \& phm. obs.: [307]; mass function suggests BH accretor: [867, 1075, 1144]; UV obs.: [179, 435]; radio obs.: [32, 670]; radio burst: [32]; quiescent ellipsoidal lt. curve: [867, 1129]; in quiescence $B=20.9, V=20.4$ : [303, 867]; secondary K0-4V: [1075]; IR lt. curve: [1144]; distance: [1144]. 
Table 1. continued

\begin{tabular}{|c|c|c|c|c|c|c|}
\hline $\begin{array}{l}\text { Source } \\
\text { Name(s) } \\
\text { Spacecraft }\end{array}$ & $\begin{array}{l}\text { type } \\
\text { Pos. }\end{array}$ & $\begin{array}{l}\mathrm{RA} \\
\mathrm{DEC} \\
l^{\mathrm{II}}, b^{\mathrm{II}}\end{array}$ & $\begin{array}{l}\text { Opt. Ctp. } \\
{[\mathrm{FC}]}\end{array}$ & $\begin{array}{l}V, B-V, U-B \\
E_{B-V}\end{array}$ & $\begin{array}{l}F_{\mathrm{x}} \\
\mu \mathrm{Jy}\end{array}$ & $\begin{array}{l}P_{\text {orb }}(\mathrm{hr}) \\
P_{\text {pulse }}(\mathrm{s})\end{array}$ \\
\hline $1254-690$ & $\mathrm{BD}$ & $\begin{array}{l}125421.0 \\
-690108\end{array}$ & GR Mus & $\begin{array}{l}19.1,0.3,-- \\
0.35\end{array}$ & 25 & 3.93 \\
\hline $\mathrm{U}, \mathrm{M}, \mathrm{A}, \mathrm{H}, \mathrm{S}, \mathrm{X}, \mathrm{G}$ & $\mathrm{o}$ & $303.5,-6.4$ & {$[463]$} & {$[927]$} & {$[247]$} & {$[247,927]$} \\
\hline
\end{tabular}

Pos.(source D): [103]; X-ray bursts, X-ray dips: [247]; X-ray spectrum: [539, 843, 1130, 1420]; X-ray/opt. obs.: [1175]; opt. burst: [843]; X-ray obs.: [1315]; opt. spectrum: [253]; opt. light curve \& spectrum: [927]; near-IR spectrum: [255].

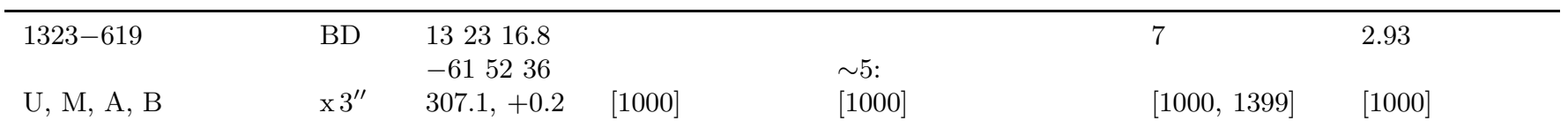

Pos.(sourceD): [1000]; X-ray obs.: [35]; X-ray bursts \& dips: [1000, 1330]; QPO: [642].

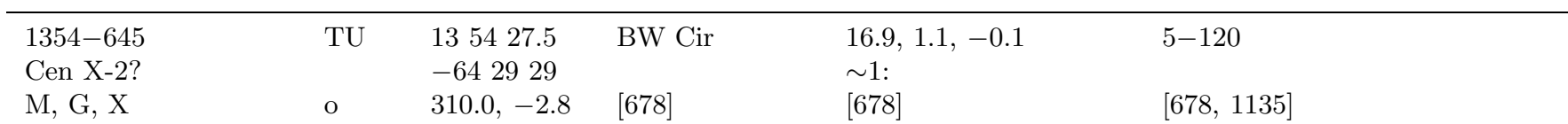

Pos.: [678]; outburst: [678, 1089]; pos. consistent with transient Cen X-2 (1967): [202, 389, 506, 813]; opt. id.: [678]; opt. sp.: [119, 253]; possible 50.0 hour period during decline: [146]; near IR phm.: [1201]; $R_{\text {quiesc }} \sim 22:$ [678]; probable radio cpt.: $[356]$.

\begin{tabular}{lllllll}
\hline $1455-314$ & TB & 145519.6 & V822 Cen & $12.8,0.05,-0.9$ & $0.1-20000$ & 15.10 \\
Cen X-4 & & -312809 & & 0.1 & & \\
V, A, Ha, AS, B, R & o & $332.2,+23.9$ & {$[141]$} & {$[84,141]$} & {$[231,1350]$} & {$[196,254,858]$}
\end{tabular}

Pos.: [103]; transient: [99, 231, 348, 658, 852]; quiescent X rays: [16, 133, 135, 1350]; opt. outburst sp. \& phm.: [141]; K5-7 secondary: [196, 858, 1347]; near-IR sp.: [255]; IR phm. \& sp. obs.: [1137]; $V_{\text {quiesc }} \sim 18.3$ : [858]; optical quiescent lt. curve.: [196, 858]; UV: [84, 859]; X-ray bursts: [67,852]; no X-ray eclipses: [1423]; radio obs.: [541, 550].

\begin{tabular}{lllllll}
\hline $1516-569$ & TBA & 151648.4 & BR Cir & $21.4,--,-$ & $5-3000$ & 398.4 \\
Cir X-1 & & -565912 & & $>1.0$ & & \\
U, M, A, H, S, AS, X & r & $322.1,+0.0$ & {$[916]$} & {$[916,1216]$} & {$[103]$} & {$[655]$}
\end{tabular}

Pos.: [9]; orbital variations: [655, 955, 1274]; X-ray obs.: [324]; X-ray spectrum: [110, 780]; Fe K line: [111]; distance: [438]; QPO: [1157, 1274, 1275]; X-ray bursts: [1276, 1277]; atoll source: [972]; opt. cpt.: [9, 916]; not the opt. cpt. in [1408]; opt. lt. curve: [916]; opt. sp.: [334, 636]; IR obs: [424, 426]; long-term weakening in $K$ band: [916]; radio obs.: [333, 516, 1217, 1408]; correlated radio/X-ray obs.: [1216]; connection SNR (?): [214]; high kick velocity: [1266]; high eccentricity: [636, 972, 1266]; VLBI obs.: [1055]; model: [942].

\begin{tabular}{|c|c|c|c|c|c|}
\hline $1524-617$ & $\mathrm{TU}$ & 152405.8 & KY TrA & $17.5 \mathrm{~B},--,--$ & $<5-950$ \\
\hline $\operatorname{Tr} A \quad X-1$ & & -614235 & ${ }^{*} \mathrm{~N}$ & 0.7 & \\
\hline A, S, R.Gr & o $3^{\prime \prime}$ & $320.3,-4.4$ & {$[940]$} & {$[940]$} & {$[103]$} \\
\hline
\end{tabular}

Pos.: [103]; X-ray outburst in 1977: [654]; ultra-soft X-ray spectrum: [1410]; X-ray obs.: [47]; hard component in quiescent X-ray spetrum: [44, 1228]; optical outburst: [940]; $V_{\text {quiesc }}>21:$ [940].

\begin{tabular}{lllllll}
\hline $1543-475$ & TU & 154333.9 & star & $14.9,0.6,--$ & $<1-15000$ & 26.95 \\
& & -473054 & & 0.7 & & \\
U, M, T, C & o & $330.9,+54$ & {$[183]$} & {$[1025,1340]$} & {$[103,676]$} & {$[984]$}
\end{tabular}

Pos.: [1025]; opt. id.: [1021, 1025]; ultra-soft X-ray spectrum, Fe K $\alpha$ emission: [200, 1211, 1340]; X-ray outbursts: [498, 676, 759, 848]; $\gamma$-ray obs.: [478, 741]; quiescent opt. phm.: [183]; quiescent $V=16.7, B=17.5$ : [183]; triple star (?): [183]; opt. sp., spectral type A2 V: [984]; mass function (BHC): [984]; distance of $9.1 \mathrm{kpc:} \mathrm{[984].}$

\begin{tabular}{|c|c|c|c|c|c|}
\hline $1543-624$ & & $\begin{array}{l}154334.1 \\
-622451\end{array}$ & ${ }^{*} 6$ & $\begin{array}{l}\geq 20(\mathrm{~B}),--,-- \\
0.5\end{array}$ & 35 \\
\hline $\mathrm{U}, \mathrm{M}, \mathrm{A}, \mathrm{H}, \mathrm{S}$, Exo & o & $321.8,-6.3$ & {$[8]$} & {$[860]$} & {$[1399$} \\
\hline
\end{tabular}

Pos.: [103]; X-ray obs.: [1164]; opt. cpt.: [860]. 
Table 1. continued

\begin{tabular}{|c|c|c|c|c|c|c|}
\hline $\begin{array}{l}\text { Source } \\
\text { Name(s) } \\
\text { Spacecraft }\end{array}$ & $\begin{array}{l}\text { type } \\
\text { Pos. }\end{array}$ & $\begin{array}{l}\mathrm{RA} \\
\mathrm{DEC} \\
l^{\mathrm{II}}, b^{\mathrm{II}}\end{array}$ & $\begin{array}{l}\text { Opt. Ctp. } \\
{[\mathrm{FC}]}\end{array}$ & $\begin{array}{l}V, B-V, U-B \\
E_{B-V}\end{array}$ & $\begin{array}{l}F_{\mathrm{x}} \\
\mu \mathrm{Jy}\end{array}$ & $\begin{array}{l}P_{\text {orb }}(\mathrm{hr}) \\
P_{\text {pulse }}(\mathrm{s})\end{array}$ \\
\hline $\mathrm{J} 1550-564$ & $\mathrm{~T}$ & $\begin{array}{l}155058.78 \\
-562835.0\end{array}$ & & $\begin{array}{l}16.6-21.4,1.35,-- \\
0.7-1.6\end{array}$ & $600-7000$ & 36.96 \\
\hline $\mathrm{X}, \mathrm{AS}, \mathrm{C}$ & o $2^{\prime \prime}$ & $325.9,-1.8$ & {$[630]$} & {$[629,630,1114]$} & {$[272,1080]$} & {$[631]$} \\
\hline \multicolumn{7}{|c|}{$\begin{array}{l}\text { Pos.: [630]; discovery: [1188]; distance } 2.5-6 \mathrm{kpc:}[1114,1194] \text {; radio counterpart: [138]; X-ray light curve: [1196]; BHC: } \\
\text { [272, 1194, 1196]; opt obs.: [160, 630, 1114]; X-ray sp.: [1196]; orbital period of } 1.398 \text { d from X-ray obs.: [271]; mass of the } \\
\text { BH: [1196]; X-ray spectral parameters: [1196]; low-freq. QPOs: [272, 1082, 1194]; higher QPO at } 182 \text { Hz: [576, 868, 1082]; } \\
\text { phase lag: [274, 1439]; very bright X-ray nova: [1080]; two different types of low freq. QPO: [1439]; a QPO at 284 Hz and } \\
\text { then settled back to } 182 \mathrm{~Hz} \text {; [576, 1081]; strong aperiodic X-ray variability: [272]; opt. flare: [1114]; sp. type K0-K5 V: [1114]. }\end{array}$} \\
\hline $1556-605$ & & $\begin{array}{l}155645.8 \\
-603552\end{array}$ & $\begin{array}{l}\mathrm{LU} \operatorname{Tr} \mathrm{A} \\
{ }^{*} \mathrm{X}\end{array}$ & $\begin{array}{l}18.6-19.2,0.45,-0.7 \\
0.6\end{array}$ & 16 & 9.1: \\
\hline $\mathrm{U}, \mathrm{M}, \mathrm{A}, \mathrm{H}, \mathrm{S}$ & o & $324.1,-5.9$ & {$[928]$} & {$[928,1127]$} & {$[928,1399]$} & {$[1170]$} \\
\hline
\end{tabular}

Pos.: [103]; opt./X-ray obs.: [928]; opt. phm.: [1127]; opt. spectrum: [253]; proposed orbital period of 9.1 hr requires confirmation: [1170].

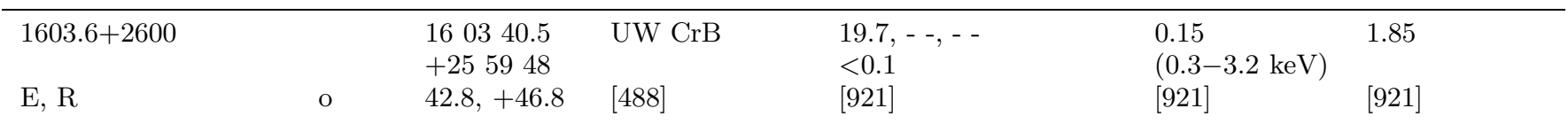

Pos.: [921]; period of a CV but opt. sp. of an LMXB: [921]; X-ray/opt. phm. obs., a BHC rather than a NS: [488].

\begin{tabular}{llll}
\hline J1603.9-7753 & TB & 160354 & 160 \\
B & x $3^{\prime}$ & $312.4,-18.7$ & {$[933]$}
\end{tabular}

Pos.: [933]; event lasted 40 s: [933]; not detected 1.1 day before: [933].

\begin{tabular}{llllll}
\hline 1608-522 & TBA & 160852.2 & QX Nor & $I \sim 18.2,-,-\cdots$ & $<1-110$ \\
& & -521743 & & 1.5 & \\
U, M, H, S, AS, C, X & o & $330.9,-0.9$ & {$[470,1382]$} & {$[470,1028]$} & {$[103]$}
\end{tabular}

Pos.: [470]; outbursts: [351, 653, 772, 813, 1264, 1461]; X-ray bursts: [68, 445, 467,935, 936, 938, 946, 947, 1028]; off-state X-ray emission: [814,1264]; X-ray sp.: [539, 908, 1108, 1245, 1420]; atoll source: [507]; kHz QPOs: [78, 877]; kHz QPOs peak separation not const.: [879]; millisecond time lag: [1364]; freq. correlated with count rate: [1457]; long-term X-ray obs., likely orbital period $4.1 \mathrm{~d}$ : [773]; $I_{\text {quiesc }}>20$ : [470]; opt./IR phm.: [1382].

\begin{tabular}{lccllll}
\hline $1617-155$ & $\mathrm{Z}$ & 161704.5 & V818 Sco & $12.2,0.2,-0.8$ & 14000 & 18.90 \\
Sco X-1 & & -153115 & & $0.15-0.3$ & & \\
U, M, A, H & o & $359.1,+23.8$ & {$[447,1115]$} & {$[397,537,1379]$} & {$[1399]$} & {$[442,727]$}
\end{tabular}

Pos.: [103]; X-ray sp.: [539, 628, 649, 650, 780, 1245, 1380, 1418]; QPO: [509, 753, 890, 1061, 1327, 1332]; kHz QPOs: [1328]; kHz QPOs peak separat. not const.: [1338]; Z source: [507, 1131]; no orb. X-ray var.: [1058]; X-ray lt. crv.: [873]; no X-ray pulsations: [531, 1449]; hard X-rays: [628, 1103, 1305]; X-ray/opt./radio obs.: [104, 139]; X-ray/opt. obs.: [18, 602, 918, 1037]; X-ray/UV obs.: [652]; X-ray/radio obs.: [553]; opt. obs.: [537]; radio obs.: [1, 548, 1387]; VLBI obs.: [102]; opt. sp.: [1122]; UV obs.: [651, 1379, 1441]; near-IR sp.: [255]; no radio lobes: [373]; $P_{\text {orb }}=3 \mathrm{hr}$ : [101]; review (1977): [911]; distance 2.8 kpc: $[102]$.

\begin{tabular}{|c|c|c|c|c|c|c|}
\hline $\begin{array}{l}1624-490 \\
\mathrm{U}, \mathrm{M}, \mathrm{A}, \mathrm{H}, \mathrm{S}, \mathrm{X}, \mathrm{B}\end{array}$ & $\begin{array}{l}\mathrm{D} \\
\times 12^{\prime \prime}\end{array}$ & $\begin{array}{l}162417.8 \\
-490446 \\
334.9,-0.3\end{array}$ & {$[1072]$} & $\begin{array}{l}\sim 7 \\
{[1404]}\end{array}$ & $\begin{array}{l}55 \\
{[1399]}\end{array}$ & $\begin{array}{l}21 \\
{[640]}\end{array}$ \\
\hline \multicolumn{7}{|c|}{ Pos.: [103]; X-ray obs.: [1185]; X-ray dips: [640, 1404]. } \\
\hline $\begin{array}{l}\text { 1627-673 } \\
\mathrm{U}, \mathrm{M}, \mathrm{A}, \mathrm{H}, \mathrm{S}, \\
\mathrm{B}, \mathrm{R}, \mathrm{AS}, \mathrm{C}, \mathrm{X}, \text { Exo }\end{array}$ & $\mathrm{P}$ & $\begin{array}{l}162714.7 \\
-672118 \\
321.8,-13.1\end{array}$ & $\begin{array}{l}\text { KZ TrA } \\
* 4 \\
{[105,633]}\end{array}$ & $\begin{array}{l}18.5,0.1,-1.2 \\
0.1 \\
{[861,1348]}\end{array}$ & $\begin{array}{l}25 \\
{[872]}\end{array}$ & $\begin{array}{l}0.69 \\
7.7 \\
{[891,943]}\end{array}$ \\
\hline
\end{tabular}

Pos.: [103]; X-ray obs.: [164, 855, 990]; X-ray pulsations: [672, 943]; X-ray spectrum: [1380]; X-ray pulse-phase spectrum: [1050, 1416]; 1000 s flaring: [762]; QPO: [1156]; aperiodic variability: [70]; cyclotron resonance feature: [979]; orbital parameters: [738]; opt. pulsations: [161, 601, 891]; opt. QPO: [161]; opt. spectrum: [253]; evolution: [1367]. 
Table 1. continued

\begin{tabular}{lllllll}
\hline $\begin{array}{l}\text { Source } \\
\text { Name(s) }\end{array}$ & type & RA & Opt. Ctp. & $V, B-V, U-B$ & $\begin{array}{l}F_{\mathrm{x}} \\
\mu \mathrm{Jy}\end{array}$ & $\begin{array}{l}P_{\text {orb }}(\mathrm{hr}) \\
P_{\text {pulse }}(\mathrm{s})\end{array}$ \\
Spacecraft & Pos. & $\begin{array}{l}\text { DEC } \\
l^{\mathrm{II}}, b^{\mathrm{II}}\end{array}$ & {$[\mathrm{FC}]$} & & $<2-1400$ \\
\hline $1630-472$ & TUD & $\begin{array}{l}163019.4 \\
\end{array}$ & & & 4.5 & {$[103]$}
\end{tabular}

Pos.: [997]; QPOs: [315, 1289]; X-ray outburst: [976]; recurrent transient ( 600 d interval): [639, 1003, 1057]; BHC: [49, 716]; X-ray dip: [1291]; Fe emission line: [273]; radio cpt.: [562]; very soft X-ray sp., hard X-ray tail: [997]; search for IR cpt.: [130].

\begin{tabular}{|c|c|c|c|c|c|c|}
\hline $\begin{array}{l}1632-477 \\
\mathrm{~K}\end{array}$ & \multirow[t]{2}{*}{$\mathrm{x} 1.1^{\prime}$} & \multicolumn{2}{|l|}{$\begin{array}{l}163246 \\
-474332 \\
336.9,-0.4\end{array}$} & & \multicolumn{2}{|l|}{13} \\
\hline \multicolumn{6}{|l|}{ Pos.: [1240]. } & \\
\hline $1636-536$ & $\mathrm{BA}$ & $\begin{array}{l}163656.4 \\
-533918\end{array}$ & $\begin{array}{l}\text { V801 Ara } \\
{ }_{*} 3\end{array}$ & $\begin{array}{l}17.5,0.7,-0.7 \\
0.8\end{array}$ & 220 & 3.80 \\
\hline $\mathrm{U}, \mathrm{M}, \mathrm{A}, \mathrm{H}, \mathrm{S}, \mathrm{X}, \mathrm{Exo}$ & $\mathrm{o}$ & $332.9,-4.8$ & {$[633]$} & {$[730,861]$} & [1399] & {$[1354]$} \\
\hline
\end{tabular}

Pos.: [103]; X-ray bursts: [114, 282, 567, 609, 752, 966, 1226, 1247, 1253, 1303, 1334, 1349, 1395]; millisecond pulsation: [1466]; spin-down: [1219]; kHz QPOs: [1433]; kHz QPO study: [880, 1364]; X-ray sp.: [539, 780, 1063, 1318, 1380, 1420]; atoll source: [507, 1131]; opt. lt. curve: [1022, 1125, 1174, 1354]; opt. cpt.: [861]; opt. bursts: [730, 853, 1023, 1024, 1125, 1298, 1304]; opt sp.: [140, 253]; reddening: [730, 1348].

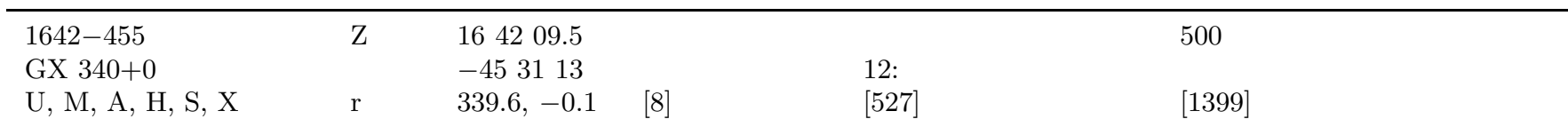

Pos.: [1033]; Z source: [507, 1131]; QPO: [753, 1032, 1327, 1353]; kHz QPO and a new broad component: [641, 645]; X-ray spectrum: [1380]; no X-ray pulsations: [1449]; radio/X-ray obs.: [971]; radio obs.: [471, 1033].

\begin{tabular}{lllllll}
\hline J1655-40 & TD & 165400.137 & ${ }^{*} 1$ & $14.2-17.3,1.0,--$ & 1600 & 62.88 \\
XN Sco 1994 & & -395044.90 & & $1.1-1.3$ & $(2-12 \mathrm{keV})$ & \\
C, AS, X, R, Gr & o & $345.0,+2.5$ & {$[30,1324]$} & {$[30,593]$} & {$[1076]$} & {$[30,1323]$}
\end{tabular}

Pos.: [30]; discovery: [1458]; not the same source of 1ES 1649-403: [69]; $\gamma$-ray obs.: [702]; absorption line in X-ray sp.: [1310]; opt. sp. obs.: [30, 80, $981,1147,1150,1202]$; the BHC mass: [31, 981, 1040, 1147, 1202, 1323, 1324, 1462]; opt. polarimetric obs.: [428, 1121]; opt. phm. obs.: [30, 31, 981, 1323, 1324]; red- and blue-shifted iron line: [36]; radial velocity curve: $[1040,1150,1202]$; inclination angle: [30, 544, 981, 1323, 1324, 1462]; repeated outbursts separated by 120 d: [1459]; opt. lt. curve: [30, 1323]; X-ray lt. curve: [739, 1268]; radio cpt.: [137]; radio flux lt. curve: [1268]; distance of 3 kpc: [30, 458, 544, 1287]; superluminal radio jets: [503, 544, 1287]; two kinds of hard X-ray outbursts: [1268]; secondary F3-F6 IV: [31, 981]; going through the three states: [878]; opt. and UV brightened: [583]; rapid var. between the opt./UV and X-ray emission, reprocession of X-ray into opt. and UV emission: [592]; multiwvl. obs.: [593, 1268]; anticorrelation of X-ray and opt. emission: [593]; anticorrelation between high accretion luminosity and radio jet ejection: [874]; time delay between the opt. and X-ray outbursts: [491, 983]; broadband (1 keV-2 MeV) X-ray sp.: [1292, 1462]; X-ray sp.: [1195]; QPO: [1083]; X-ray dips: $[717,720]$.

\begin{tabular}{lllllll}
\hline $1656+354$ & $\mathrm{P}$ & 165601.7 & HZ Her & $13.0-14.6,-0.2,-0.9$ & $15-50$ & 40.80 \\
Her X-1 & & +352505 & & $<0.05$ & 1.24 \\
U, M, A, H, G, X, B, & o & $58.2,+37.5$ & {$[326,447]$} & {$[100,483]$} & {$[872,1448]$} & {$[297,943]$}
\end{tabular}

Pos.: [103]; X-ray obs.: [299, 732, 1004, 1133, 1152]; X-ray pulse profile, NS precession (?): [1198, 1199, 1299]; orb. period decrease: [298]; X-ray sp.: [280, 780, 895, 975,9 78, 1380, 1416]; cyclotron line: [894, 1297, 1302]; X-ray pulse-phase sp.: [344, 1200]; aperiodic var.: [70, 71]; X-ray behaviour chaotic: [958, 1372]; 35-day cycle: [964]; X-ray eclipse: [295]; X-ray orbit: [297]; opt. It curve: [6, 25, 26, 86, 784]; 35 day effect opt. It curve: [100, 184, 296, 417]; extended low state: [995]; low-state opt. It curve: [301, 906]; off-state opt. lt curve: [586, 638]; opt. sp.: [256, 257, 258, 688, 1091]; opt. radial-velocity curve: [590]; near-IR sp.: [255]; opt. pulsations: [889, 892, 893]; 35-day effects opt. sp.: [590]; optical polarimetry: [338]; UV/opt. obs.: [1374]; UV: [5, 180, 335, 483, 584, 585]; EUV: [92, 733]; disk precession (?): [686, 1038, 1039, 1067]; multiwvl. obs.: [91, 1381]; evolution: [1367]; strange star: [764]; not a strange star: [787]. 
Table 1. continued

\begin{tabular}{|c|c|c|c|c|c|c|}
\hline $\begin{array}{l}\text { Source } \\
\text { Name(s) } \\
\text { Spacecraft }\end{array}$ & $\begin{array}{l}\text { type } \\
\text { Pos. }\end{array}$ & $\begin{array}{l}\mathrm{RA} \\
\mathrm{DEC} \\
l^{\mathrm{II}}, b^{\mathrm{II}}\end{array}$ & $\begin{array}{l}\text { Opt. Ctp. } \\
{[\mathrm{FC}]}\end{array}$ & $\begin{array}{l}V, B-V, U-B \\
E_{B-V}\end{array}$ & $\begin{array}{l}F_{\mathrm{x}} \\
\mu \mathrm{Jy}\end{array}$ & $\begin{array}{l}P_{\text {orb }}(\mathrm{hr}) \\
P_{\text {pulse }}(\mathrm{s})\end{array}$ \\
\hline $1658-298$ & TBD & $\begin{array}{l}165855.4 \\
-295228\end{array}$ & $\begin{array}{l}\text { V2134 Oph } \\
{ }^{*} \mathrm{~T}\end{array}$ & $\begin{array}{l}18.3,0.45,-0.4 \\
0.3\end{array}$ & $<5-80$ & 7.11 \\
\hline $\mathrm{A}, \mathrm{H}, \mathrm{X}$ & o & $353.8,+7.3$ & {$[330]$} & {$[330,954]$} & [103] & {$[228]$} \\
\hline
\end{tabular}

Pos.: [103]; X-ray dips: [226, 227]; X-ray bursts: [225, 746, 749, 1153]; opt. spectrum: [20, 140, 253]; opt. phm.: [1385, 1386]; $\mathrm{X}$-ray reappearance after 21 yr quiescence: [1386].

\begin{tabular}{lllllll}
\hline $1659-487$ & TU & 165902.0 & V821 Ara & $15.5,0.8,-0.1$ & $1.5-900$ & 14.83 \\
GX339-4 & & -484307 & ${ }^{*}$ V & 1.1 & & \\
U, M, A, H, K, B, X, & o & $338.9,-4.3$ & {$[122,330]$} & {$[252,800,1348]$} & {$[603,912]$} & {$[122,582]$} \\
C & & & & &
\end{tabular}

Pos.: [103]; high-low-off X-ray states/optical state correlated: [603, 800, 810, 912, 925, 926]; X-ray/opt. obs.: [687]; a state transition: [76]; Fe line at $6.4 \mathrm{keV}$ : [1442]; a sequence of X-ray outbursts: [1104]; off state $B>21$ : [589, 599]; radio/X-ray/ $\gamma$ ray obs.: [1192]; X-ray QPO: [451, 912, 1210]; rapid X-ray var.: [70, 789, 913, 1191]; hard X rays: [317, 369, 1295]; X-ray halo: [1053]; X-ray spectrum: [1380]; X-ray obs.: [448, 502, 963, 1470]; long-term radio/soft-/hard X-ray campaign: [235]; very soft X-ray sp. component: [800, 912]; opt. QPO: [607, 924, 925, 1209]; opt. spectrum: [237, 252, 253, 330, 464, 1193, 1204]; opt. millisec. pulsations (?): [606]; near-IR sp.: [255]; radio obs.: [357, 495]; radio emission correlated with hard X-ray emission: [361].

\begin{tabular}{lllllll}
\hline $1702-363$ & $\mathrm{Z}$ & 170222.9 & ${ }^{*} 6$ & $18.6,1.5,--$ & 825 & $21.85-22.5$ \\
GX349+2 & & -362120 & & $1.3:$ & & \\
U, M, A, H, S, X & $\mathrm{r}$ & $349.1,+2.7$ & {$[234,1026]$} & {$[234,1026]$} & {$[1399]$} & {$[55,1383,1384]$}
\end{tabular}

Pos.: [234]; Z source: [507]; odd Z source, similiar to bright atoll source: [710]; X-ray obs.: [539, 1131]; X-ray spectrum: [709, 780, 1380, 1420]; QPO: [753, 1045, 1327]; kHz QPOs: [1468]; orbital period of $14.9 \mathrm{~d}(?)$ : [55, 1207]; radio obs.: $[234,416,471]$.

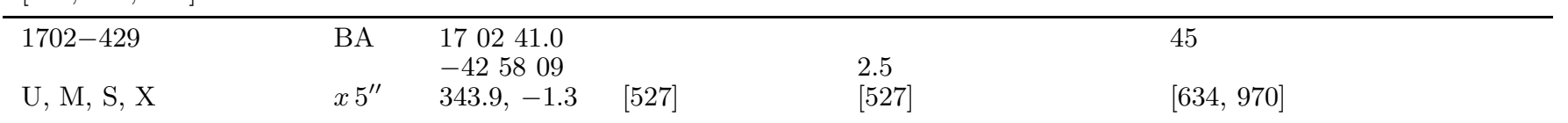

Pos.: [527]; atoll source: [970]; X-ray bursts: [798, 817, 970, 1008, 1248]; kHz QPOs: [817]; radio obs.: [471].

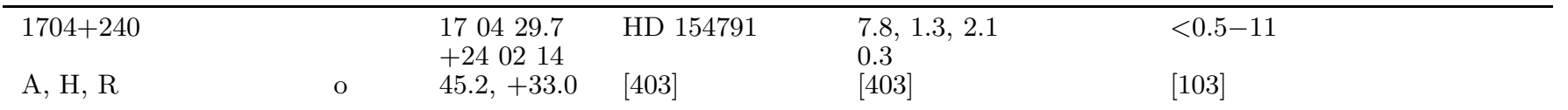

Pos.: [103]; opt. sp. \& phm.: [1288]; sp. type of opt. cpt. M 3 II: [403]; opt. sp.: [413]; UV: [403]; HMXB (?): [279].

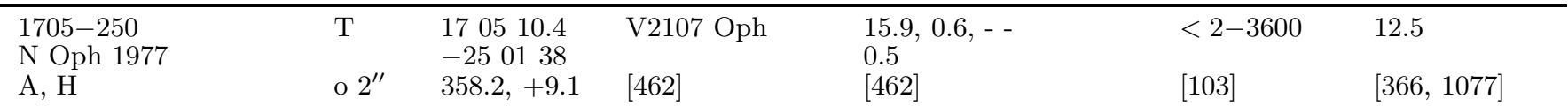

Pos.: [103]; X-ray outburst 1977: [1401]; very soft X-ray spectrum with hard tail: [233, 1444]; opt. sp. \& phm.: [1077]; $B_{\text {quiesc }} \sim 21:$ [462]; $P_{\text {orb }}=16.8$ h: [834]; large mass function: [366]; sp. type of K3-7: [366, 1077]; distance: [834, 1077].

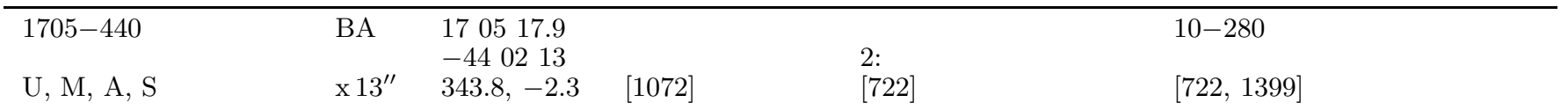

Pos.: [103]; atoll source: [507]; X-ray spectrum: [1130, 1380]; kHz QPO: [379]; hard lag: [381]; X-ray bursts: [446, 722]; X-ray power spectra: [723]; unusually hard sp.: [77]; X rays: [1131]; long-term on-off (223d?): [1056, 1057]; radio obs.: [333].

\begin{tabular}{llll}
\hline $1708-408$ & & 170853 & 32 \\
U, M, A, H & $\mathrm{x} 1^{\prime}$ & $346.3,-0.9$ & {$[611]$} \\
Pos.: $[611]$. & & & \\
\hline J1709-267 & TB & 170930.2 & 150 \\
X, R, B & $\mathrm{x} 2^{\prime}$ & $357.5,+7.9$ & {$[825]$}
\end{tabular}

Pos.: [1373]; X-ray nova: [825]; the same source as 1RXS J170930.2-263927: [218]; type-I X-ray burster: [218]; possible radio cpt. [545] out of the ROSAT error: [218]; a possible LMXB containing a NS: [218]; X-ray light curve: [218]; distance: [218]. 
Table 1. continued

\begin{tabular}{|c|c|c|c|c|c|c|}
\hline $\begin{array}{l}\text { Source } \\
\text { Name(s) } \\
\text { Spacecraft }\end{array}$ & $\begin{array}{l}\text { type } \\
\text { Pos. }\end{array}$ & $\begin{array}{l}\mathrm{RA} \\
\mathrm{DEC} \\
l^{\mathrm{II}}, b^{\mathrm{II}}\end{array}$ & $\begin{array}{l}\text { Opt. Ctp. } \\
{[\mathrm{FC}]}\end{array}$ & $\begin{array}{l}V, B-V, U-B \\
E_{B-V}\end{array}$ & $\begin{array}{l}F_{\mathrm{x}} \\
\mu \mathrm{Jy}\end{array}$ & $\begin{array}{l}P_{\text {orb }}(\mathrm{hr}) \\
P_{\text {pulse }}(\mathrm{s})\end{array}$ \\
\hline J1710-281 & $\mathrm{TB}(?)$ & $\begin{array}{l}171012.3 \\
-280754\end{array}$ & & & 2 & \\
\hline $\mathrm{X}, \mathrm{R}$ & $\mathrm{x}$ & $356.4,+6.9$ & & & {$[816]$} & \\
\hline
\end{tabular}

Pos.: [816]; type I X-ray burst (?): [819]; the same source of 1RXS J171012.3-280754: [816].

\begin{tabular}{llll}
\hline $1711-339$ & $\mathrm{~T}$ & 171101.3 & $16-130$ \\
& & -335932 \\
$\mathrm{H}, \mathrm{S}, \mathrm{R}$ & $\mathrm{x} 40^{\prime \prime}$ & $352.1,+2.7 \quad[453]$ & {$[103]$}
\end{tabular}

Pos.: [103]; radio obs.: [453]; recurrent X-ray emission: [1074].

\begin{tabular}{llll}
\hline J1712.6-3739 & TB & 171234 & 34 \\
& & -3738.6 & $(2-9 \mathrm{keV})$ \\
$\mathrm{B}$ & $\mathrm{x} 3^{\prime}$ & 348.9, & {$[619]$} \\
& & +0.94 &
\end{tabular}

Pos.: [619]; type I X-ray burst with peak flux 1.7 Crab units $(2-26 \mathrm{keV})$ : [220].

\begin{tabular}{|c|c|c|c|c|c|c|}
\hline $\begin{array}{l}1715-321 \\
\mathrm{M}, \mathrm{A}, \mathrm{H}, \mathrm{S}\end{array}$ & $\begin{array}{l}\mathrm{B} \\
\times 23^{\prime \prime}\end{array}$ & $\begin{array}{l}171532.3 \\
-320734 \\
354.1,+3.1\end{array}$ & {$[1072]$} & & \multicolumn{2}{|l|}{28} \\
\hline \multicolumn{7}{|c|}{ Pos.: [103]; X-ray bursts: $[569,796,1270]}$. \\
\hline $\begin{array}{l}1716-249 \\
\text { XN Oph } 1993 \\
\text { Gr, K, C, AS }\end{array}$ & $\begin{array}{l}\mathrm{T} \\
\mathrm{r} 0.5^{\prime \prime}\end{array}$ & $\begin{array}{l}171632.52 \\
-245801.1 \\
359.8,+7.6\end{array}$ & $\begin{array}{l}\text { V2293 Oph } \\
{[305,838]}\end{array}$ & $\begin{array}{l}16.65-21,0.9,-- \\
0.9 \\
{[305]}\end{array}$ & $\begin{array}{l}1500 \\
(20-100 \mathrm{keV}) \\
{[501]}\end{array}$ & 14.7 \\
\hline
\end{tabular}

Pos.: [901]; discovery: [33, 500]; GRO source J1719-24: [500]; BHC: [838]; opt. and radio counterpart: [305]; opt. photometric and sp. obs.: [305, 838]; distance $\sim 2.4$ kpc: [305]; sp. type $\mathrm{K}$ or later: [305]; X-ray light curve: [501, 555, 1085]; QPOs: [1321, 1322]; hard X-ray lags: [1326]; broadband X-ray sp.: [1085]; X-ray and radio flare: [555].

\begin{tabular}{llll}
\hline J171824.2 & B & 171824.13 & 1400 \\
-402934 & & -402930.4 \\
R, B & $x 15.7$ & $347.3,-1.6$ & {$[931]$}
\end{tabular}

Pos.: [931]; distance of 6.5 kpc: [662]; no optical counterpart to be identified: [931]; type I X-ray burst: [662].

\begin{tabular}{llll}
\hline J1723-376 & TB & 172338 & 70 \\
$\mathrm{X}$, AS & & -3739.7 & [821] \\
& & 350.2, & -0.86
\end{tabular}

Pos.: [828]; QPO at $816 \mathrm{Hz:}$ [821]; type I X-ray burst: [828].

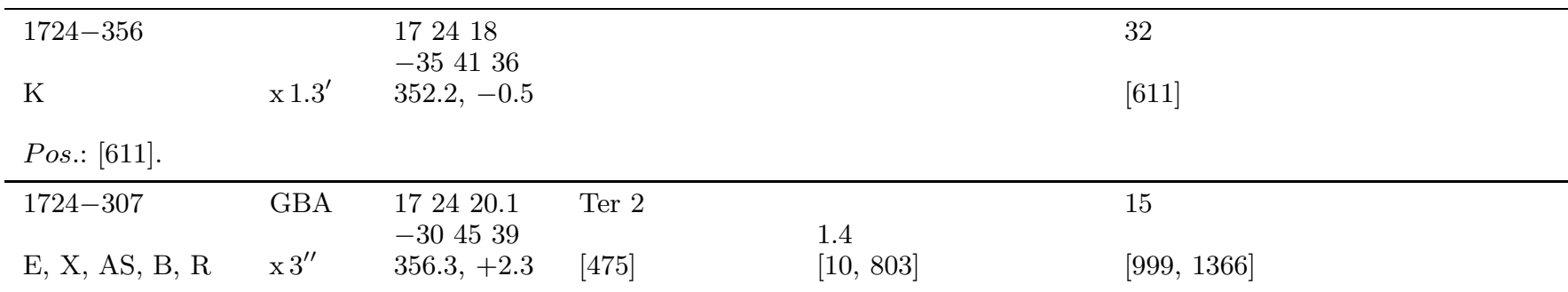

Pos.: [475, 525]; X-ray obs.: [52, 480, 969]; Fe line: [53]; opt. study globular cluster: [203]; cluster metal rich: [10]; structure gl. cl.: [528]; X-ray bursts: [474, 914, 1249]; hard X-ray obs.: [43]; IR obs.: [203]; radio/X-ray obs.: [253]; radio obs.: [471].

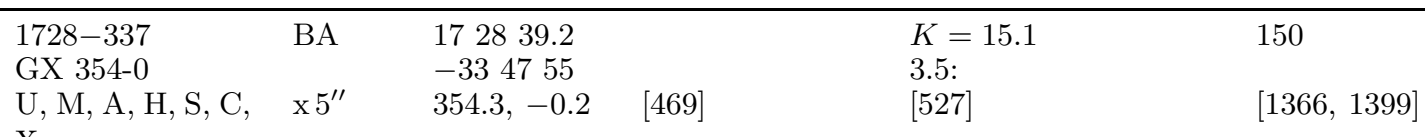

$\mathrm{X}$

Pos.: [525]; atoll source: [507]; not in globular cl.: [469, 1344]; X-ray bursts: [56, 386, 565, 566, 570, 571, 661, 1008]; QPO study: [376, 875]; kHz QPO and $2.75 \mathrm{~ms}$ pulsation: [1221, 1223]; X rays: [213, 1131]; X-ray spectrum: [1380]; radio \& IR cpt.: [832]; radio obs.: [471]; a strange star: [766]. 
Table 1. continued

\begin{tabular}{|c|c|c|c|c|c|c|}
\hline $\begin{array}{l}\text { Source } \\
\text { Name(s) } \\
\text { Spacecraft }\end{array}$ & $\begin{array}{l}\text { type } \\
\text { Pos. }\end{array}$ & $\begin{array}{l}\text { RA } \\
\mathrm{DEC} \\
l^{\mathrm{II}}, b^{\mathrm{II}}\end{array}$ & $\begin{array}{l}\text { Opt. Ctp. } \\
{[\mathrm{FC}]}\end{array}$ & $\begin{array}{l}V, B-V, U-B \\
E_{B-V}\end{array}$ & $\begin{array}{l}F_{\mathrm{x}} \\
\mu \mathrm{Jy}\end{array}$ & $\begin{array}{l}P_{\text {orb }}(\mathrm{hr}) \\
P_{\text {pulse }}(\mathrm{s})\end{array}$ \\
\hline $\begin{array}{l}1728-169 \\
\text { GX } 9+9 \\
U, M, A, H, S\end{array}$ & $\begin{array}{l}\mathrm{A} \\
\mathrm{O}\end{array}$ & $\begin{array}{l}172850.2 \\
-165532 \\
8.5,+9.0\end{array}$ & $\begin{array}{l}\text { V2216 Oph } \\
{[328,447]}\end{array}$ & $\begin{array}{l}16.8,0.3,-0.7 \\
0.3 \\
{[954,1123]}\end{array}$ & $\begin{array}{l}300 \\
{[1399]}\end{array}$ & $\begin{array}{l}4.20 \\
{[529,1123]}\end{array}$ \\
\hline \multicolumn{7}{|c|}{ Pos.: [103]; X-ray obs.: $[539,650,1131]$; X-ray sp.: $[780,1376,1380]$; opt. cpt.: $[253,288]$; near-IR sp.: [255]; radio obs.: [333] } \\
\hline $\begin{array}{l}1728-247 \\
\text { GX } 1+4 \\
\mathrm{U}, \mathrm{M}, \mathrm{A}, \mathrm{H}, \mathrm{S}\end{array}$ & $\mathrm{P}$ & $\begin{array}{l}172857.9 \\
-244235 \\
1.9,+4.8\end{array}$ & $\begin{array}{l}\text { V2116 Oph } \\
{ }^{*} \mathrm{GF} \\
{[328]}\end{array}$ & $\begin{array}{l}19.0,--,-- \\
1.7 \mathrm{P} \\
{[288,289]}\end{array}$ & $\begin{array}{l}100 \\
{[1399]}\end{array}$ & $\begin{array}{l}340 \mathrm{~d} ? \\
114 \\
{[276,943]}\end{array}$ \\
\hline
\end{tabular}

Pos.: [103, 1167]; X-ray obs.: [286, 690, 728, 1054]; X-ray pulsations and period history: [318, 454, 744, 801, 917, 943, 1111]; Xray spectrum: [398,1094]; X-ray flare: [421]; magnetic field: [264]; correlation of torque and luminosity: [165, 1009]; spin down: [1068]; X-ray correlated $\mathrm{H} \alpha$ flare: [804]; 303.8 d periodicity: [1035]; opt./X-ray sp.: [456]; X-ray pulse phase spectroscopy: [1416]; opt. cpt. M 6 giant: [253, 289]; opt. pulsation with 124 s: [626]; GX 1+4 unlikely to be source of 511 keV emission: [856, 1015]; opt./IR/X-ray obs.: [163]; opt. phm.: [310, 625]; opt. sp.: [455]; IR obs.: [166, 425, 427].

\begin{tabular}{llll}
\hline $1730-312$ & $\mathrm{~T}$ & 1730.3 & 900 \\
$\mathrm{~K}, \mathrm{Gr}$ & $\mathrm{x} 2^{\prime}$ & $356.3,+1.6$ & {$[94]$}
\end{tabular}

Pos.: [93]; discovery: [93, 206]; hard X-ray obs.: [1360]; possibly a BH X-ray nova: [94, 1360]; X-ray sp.: [1360]; high resolution X-ray light curve: [1360].

\begin{tabular}{llllll}
\hline $1730-335$ & GBT & 173006.6 & Lil 1 & & $<0.1-200$ \\
Rapid Burster & & -332113 & & $3.0:$ & \\
S, X, AS, Exo & $\mathrm{x} 3^{\prime \prime}$ & $354.8,-0.2$ & {$[475,767]$} & {$[10,803]$} & {$[465,1215]$}
\end{tabular}

Pos.: [475, 525]; X-ray obs.: [15, 1106]; opt./IR obs. glob. cluster: [528, 682]; very metal rich gl. cluster: [10]; recurrent transient (0.5 year?): [468, 742]; type II bursts: [568, 745]; type II bursts behave as relaxation oscillator: [745, 829, 1215, 1346]; type II burst profiles: [776, 1260, 1272]; pre- and post-burst dips: [829, 1215]; type II burst patterns: [41, 708, 829, 944, 1215]; type II burst spectra: [667, 707, 754, 777, 829, 1260]; type I bursts: [41, 568, 708]; radio/IR/X-ray obs.: [729]; QPO: [320, 754, 775, 1215, 1269]; a likely radio cpt.: [919]; comparison with GRO J1744-28: [685, 757]; radio obs.: [471].

\begin{tabular}{llll}
\hline $1730-220$ & $\mathrm{~T}$ & 173056 & $<10-130$ \\
$\mathrm{U}$ & $\mathrm{x} 1.7^{\prime}$ & $4.5,+5.9$ & {$[230]$}
\end{tabular}

Pos.: [384]; X-ray outburst in 1972, relatively soft X-ray spectrum: [230].

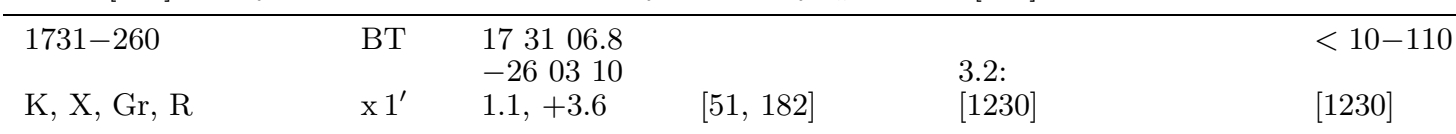

Pos.: [1230]; X-ray obs.: [46, 51, 53]; J2000 pos. with 10.1" error (17 34 13.5, -26 05 16.8): [51]; X-ray bursts: [1230, 1232]; hard X-ray sp.: [45]; kHz QPOs: [1424]; 3.8 or $1.91 \mathrm{~ms}$ pulsation: [1186, 1424]; IR phm.: [51]; two objects in [182] not its cpt.: [51].

\begin{tabular}{|c|c|c|c|c|c|}
\hline $1732-304$ & GB & $\begin{array}{l}173234.8 \\
-302703\end{array}$ & Ter 1 & $1.5-2.48$ & 10 \\
\hline Ha, B, Gr, H, R, Exo & $x 8^{\prime \prime}$ & $357.6,+0.9$ & {$[797]$} & {$[10,988,1405]$} & {$[999,1166,1366]$} \\
\hline
\end{tabular}

Pos.: [999]; moderately rich globular cluster: [10]; X-ray bursts: [608, 797]; X-ray obs.: [98, 481, 1166]; opt. phm. \& distance: [988]; radio cpt.: [832].

\begin{tabular}{|c|c|c|c|}
\hline $1732-273$ & TU & $\begin{array}{l}173254 \\
-272342\end{array}$ & $<5-50$ \\
\hline $\mathrm{K}, \mathrm{G}$ & $\mathrm{x} 1^{\prime}$ & $0.16,+2.59 \quad[182]$ & {$[612,792]$} \\
\hline
\end{tabular}

Pos.: [612, 1240]; position consistent with that of GS 1734-275 \& RX J1735.9-2726: [155, 792, 1455]; very soft X-ray sp.: [792].

\begin{tabular}{|c|c|c|c|}
\hline $1734-292$ & $\begin{array}{l}173414 \\
-290902\end{array}$ & & 3.4 \\
\hline Gr & $358.84,+1.4$ & {$[182]$} & {$[1019]$} \\
\hline
\end{tabular}

Pos.: [1240]; X-ray observations: [1019, 1020]; outburst of hard X rays: [204]. 
Table 1. continued

\begin{tabular}{|c|c|c|c|c|c|c|}
\hline $\begin{array}{l}\text { Source } \\
\text { Name(s) } \\
\text { Spacecraft }\end{array}$ & $\begin{array}{l}\text { type } \\
\text { Pos. }\end{array}$ & $\begin{array}{l}\mathrm{RA} \\
\mathrm{DEC} \\
l^{\mathrm{II}}, b^{\mathrm{II}}\end{array}$ & $\begin{array}{l}\text { Opt. Ctp. } \\
{[\mathrm{FC}]}\end{array}$ & $\begin{array}{l}V, B-V, U-B \\
E_{B-V}\end{array}$ & $\begin{array}{l}F_{\mathrm{x}} \\
\mu \mathrm{Jy}\end{array}$ & $\begin{array}{l}P_{\text {orb }}(\mathrm{hr}) \\
P_{\text {pulse }}(\mathrm{s})\end{array}$ \\
\hline SL, X, R, AS, B & $\begin{array}{l}\mathrm{B} \\
\times 2^{\prime}\end{array}$ & $\begin{array}{l}173508 \\
-265834 \\
0.78,+2.40\end{array}$ & & & {$[1166]$} & \\
\hline \multicolumn{7}{|c|}{ Pos.: [1166]; X-ray obs.: [285, 434]; type I bursts: [61]; hard X-ray emission: [53]; rapid X-ray var.: [1427] } \\
\hline $1735-444$ & $\mathrm{BA}$ & $\begin{array}{l}173519.3 \\
-442520\end{array}$ & $\begin{array}{l}\text { V926 Sco } \\
{ }^{*} 5\end{array}$ & $\begin{array}{l}17.5,0.2,-0.8 \\
0.15\end{array}$ & 160 & 4.65 \\
\hline $\begin{array}{l}\mathrm{U}, \mathrm{M}, \mathrm{A}, \mathrm{H}, \mathrm{S}, \mathrm{B}, \\
\mathrm{X}\end{array}$ & $\mathrm{O}$ & $346.1,-7.0$ & {$[633]$} & {$[492,633,1348]$} & [1399] & {$[1319]$} \\
\hline
\end{tabular}

Pos.: [103]; atoll source: [507]; X-ray obs.: [539, 1131]; X-ray spectrum: [1380]; QPO: [1029]; kHz QPOs: [380, 1436]; opt. cpt.: [861]; opt. It curve: [236, 1177, 1319]; opt. spectrum: [140, 253, 862, 1172, 1176]; near-IR sp.: [255]; opt./X-ray variability: [238, 1177]; UV: [492]; X-ray bursts: [246, 750, 1351]; optical bursts: [473, 863]; opt. phm. and flare: [79]; radio obs.: [333, 471].

\begin{tabular}{llll}
\hline $1735-28$ & $\mathrm{~T}$ & 1735.4 & $<0.4-565$ \\
$\mathrm{U}, \mathrm{M}$ & $\mathrm{x} 7^{\prime}$ & $359.6,+1.56$ & {$[230,1166]$}
\end{tabular}

Pos.: [384]; transient: [668]; soft X-ray spectrum: [230].

\begin{tabular}{llll}
\hline $1736-297$ & 173621 & 2 \\
& & -294150 \\
$\mathrm{Gr}$ & $\mathrm{x} 1^{\prime}$ & $358.63,+0.7 \quad[182]$ & {$[725]$}
\end{tabular}

Pos.: [1240]; X-ray observation: [725, 1019, 1020]; not sure if the same source of J1739.5-2942 listed in HMXB: [931].

\begin{tabular}{llll}
\hline $1737-31$ & $\mathrm{~T}$ & 1736.7 & 26 \\
& & -3057 \\
Gr, C, X, AS, B & $\mathrm{x} 3^{\prime}$ & $357.3,+0.58$ & {$[822]$}
\end{tabular}

Pos.: [1244]; discovery: [1244]; more accurate J2000 pos. (17 40 09, -31 02.4) with error box 0.5': [1309]; X-ray light curve: [267,1296]; X-ray sp.: [267, 1296]; X-ray outburst: [267, 822]; hard sp. similar to low state sp. of BHCs: [266]; possible X-ray nova and BHC: [266, 267, 1244, 1296]; not in a soft state during outburst: [267]; distance of 8.5 kpc (?): [1244]; LMXB ?.

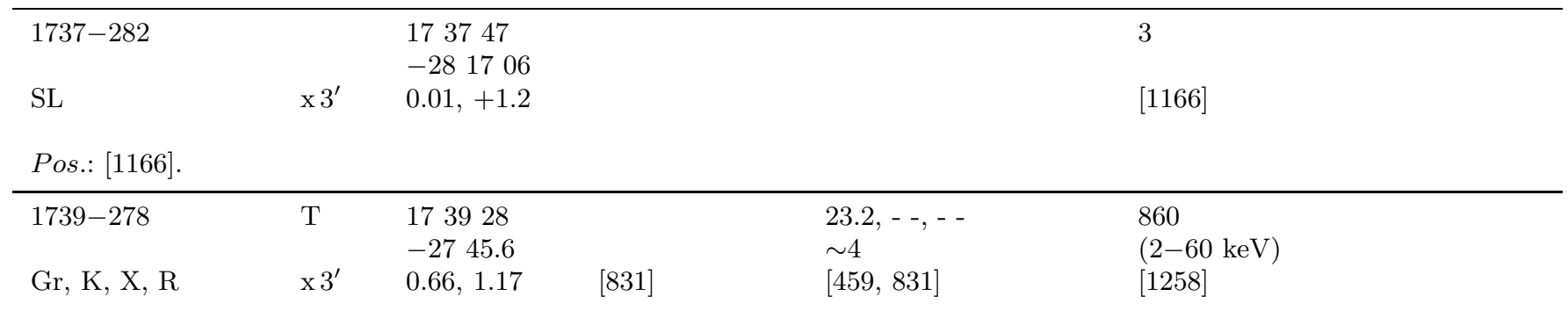

Pos.: [1016]; discovery: [1016]; radio counterpart: [556]; J2000 opt. position (17 $\left.4240.06 \pm 0.6^{\prime \prime},-274453.2 \pm 0.8^{\prime \prime}\right):$ [831]; near-IR phm. obs.: [831]; a middle G to early $\mathrm{K}$ or normal B type massive star: [831]; a BH and low-mass sys.: [97]; X-ray light curve and sp.: [97, 1361]; X-ray sp. analysis: [95, 96]; opt. and near IR counterpart in coincidence with the radio pos.: [902]; BH X-ray nova: [95, 1016, 1361]; QPO at 5 Hz: [97]; distance of $8.5 \mathrm{kpc}$ (?): [831].

\begin{tabular}{llll}
\hline $1739-304$ & & 173931 \\
& & -302929 \\
$\mathrm{~K}$ & $\mathrm{x} 1.6^{\prime}$ & $358.33,-0.3$ & 9 \\
Pos.: [1240]. & & & {$[611]$} \\
\hline $1740-294$ & $\mathrm{~T}(?)$ & 1740.6 & 30 \\
GC X-4 & $\mathrm{x} 1.2^{\prime}$ & $359.36,+0.1$ & {$[262]$} \\
& & \\
Pos.: $[262]$. & & \\
\hline
\end{tabular}


Table 1. continued

\begin{tabular}{|c|c|c|c|c|c|c|}
\hline $\begin{array}{l}\text { Source } \\
\text { Name(s) } \\
\text { Spacecraft }\end{array}$ & $\begin{array}{l}\text { type } \\
\text { Pos. }\end{array}$ & $\begin{array}{l}\mathrm{RA} \\
\mathrm{DEC} \\
l^{\mathrm{II}}, b^{\mathrm{II}}\end{array}$ & $\begin{array}{l}\text { Opt. Ctp. } \\
{[\mathrm{FC}]}\end{array}$ & $\begin{array}{l}V, B-V, U-B \\
\mathrm{E}_{B-V}\end{array}$ & $\begin{array}{l}F_{\mathrm{x}} \\
\mu \mathrm{Jy}\end{array}$ & $\begin{array}{l}P_{\text {orb }}(\mathrm{hr}) \\
P_{\text {pulse }}(\mathrm{s})\end{array}$ \\
\hline $1740.7-2942$ & & $\begin{array}{l}174042.9 \\
-294326\end{array}$ & & & $4-30$ & \\
\hline $\mathrm{E}, \mathrm{X}, \mathrm{C}, \mathrm{R}, \mathrm{AS}, \mathrm{Gr}$ & $\mathrm{x} 12^{\prime \prime}$ & $359.12,-0.1$ & {$[734,882,1169]$} & & {$[1166,1168,1233]$} & \\
\hline
\end{tabular}

Pos.: [1169]; more accurate J2000 pos.: [1162]; X-ray obs.: [59, 205, 646, 791, 1155, 1190]; long-term X rays: [245]; hard X-ray sp. (BHC): [666, 805, 1169, 1233, 1236, 1238]; source of $511 \mathrm{keV}$ annihilation line from GC region: [1015]; radio obs.: [899, 1062, 1071]; repeated outbursts of hard emission: [244]; X-ray/VLA obs.: [518]; VLA obs.: [3]; not in molecular cloud: [208, 1110]; double-sided radio jet: [900]; relation with filamentary radio emission (?): [449, 731]; near IR: [490]; accretion from ISM (?): [34, 899].

\begin{tabular}{|c|c|c|}
\hline $1741.2-2859$ & $\begin{array}{l}1741 \quad 14.7 \\
-285930\end{array}$ & $<1.5-300$ \\
\hline G & $359.8,+0.18$ & [909] \\
\hline
\end{tabular}

Pos.: [909]; alternative position $\mathrm{RA}=174139.8, \mathrm{DEC}=-2849$ 24: [909].

\begin{tabular}{lllll}
\hline $1741-293$ & TB & 174138 & $<5-26$ \\
MXB1743-29? & & -291953 \\
K, B, X & $x 1^{\prime}$ & $359.5,-0.07$ & {$[182]$} & {$[612]$}
\end{tabular}

Pos.: [612]; X-ray bursts (MXB1743-29?): [612, 748].

\begin{tabular}{llll}
\hline $1741-322$ & TU & 174146 & $<2-770$ \\
$\mathrm{H}$ & $\mathrm{x} 20^{\prime \prime}$ & $357.1,-1.6$ & {$[103,1168,1448]$}
\end{tabular}

Pos.: [1448]; transnt.: [329, 482, 653, 1447]; ultra-soft X-ray sp.: [1411]; hard X-ray excess: [233]; also known as 1743-32: [1448].

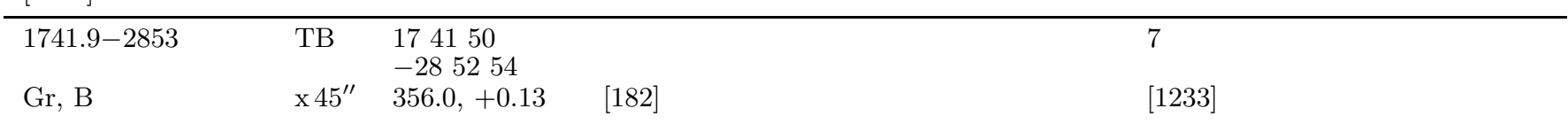

Pos.: [1020]; X-ray observation: [1237, 1240]; type I bursts: [219].

\begin{tabular}{lll}
\hline $1742-326$ & 174212 & $<2-3$ \\
Exo & -324028 & {$[1168,1400]$}
\end{tabular}

Pos.: [1400]; X-ray spectrum: [1455].

\begin{tabular}{|c|c|c|c|}
\hline $\begin{array}{l}1742.2-2857 \\
\mathrm{E}\end{array}$ & $\mathrm{x} 1^{\prime}$ & $\begin{array}{l}174215.9 \\
-285749 \\
359.9,+0.01\end{array}$ & $\begin{array}{l}0.1 \\
(0.9-4.0 \mathrm{keV}) \\
{[1403]}\end{array}$ \\
\hline Pos.: [1403]. & & & \\
\hline $\begin{array}{l}1742-294 \\
\text { GC X-2 }\end{array}$ & $\mathrm{B}(\mathrm{T} ?)$ & $\begin{array}{l}174226 \\
-2926\end{array}$ & 45 \\
\hline
\end{tabular}

Pos.: [262]; 1" error J2000 pos.: [1162]; X-ray obs.: [207]; type-I bursts: [747, 1020]; in error boxes of MXB1743-29 and MXB1742-29: [747].

\begin{tabular}{lcccc}
\hline $1742-289$ & $\mathrm{~TB}(?)$ & 174226.3 & & $<9-2000$ \\
$\mathrm{~A}$ & $\mathrm{r} 3^{\prime \prime}$ & -285957 & & \\
& & & &
\end{tabular}

Pos.: [103]; transient: [112, 262, 350, 1064, 1403]; X-ray bursts (MXB1743-29?): [747]; radio obs.: [290]; possible optical counterpart (K dwarf): [941].

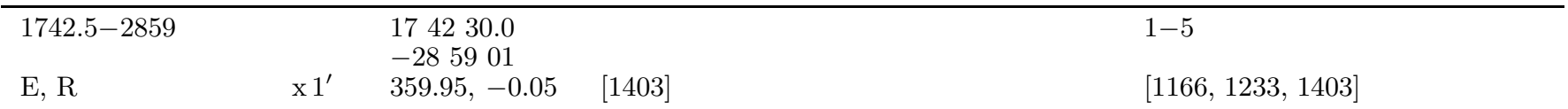

Pos.: [1403]; Sgr A west (?): [1403]; in error box MXB1743-29: [747]; X-ray obs.: [1051]. 
Table 1. continued

\begin{tabular}{|c|c|c|c|c|c|c|}
\hline $\begin{array}{l}\text { Source } \\
\text { Name(s) } \\
\text { Spacecraft }\end{array}$ & $\begin{array}{l}\text { type } \\
\text { Pos. }\end{array}$ & $\begin{array}{l}\mathrm{RA} \\
\mathrm{DEC} \\
l^{\mathrm{II}}, b^{\mathrm{II}}\end{array}$ & $\begin{array}{l}\text { Opt. Ctp. } \\
{[\mathrm{FC}]}\end{array}$ & $\begin{array}{l}V, B-V, U-B \\
E_{B-V}\end{array}$ & $\begin{array}{l}F_{\mathrm{x}} \\
\mu \mathrm{Jy}\end{array}$ & $\begin{array}{l}P_{\text {orb }}(\mathrm{hr}) \\
P_{\text {pulse }}(\mathrm{s})\end{array}$ \\
\hline $\begin{array}{l}1742.5-2845 \\
\text { E }\end{array}$ & $\mathrm{x} 1^{\prime}$ & $\begin{array}{l}174232.5 \\
-284544 \\
0.14,+0.06\end{array}$ & [1403] & & $\begin{array}{l}0.15-26 \\
(0.9-4.0 \mathrm{keV}) \\
{[1403]}\end{array}$ & \\
\hline Pos.: [1403]. & & & & & & \\
\hline $\begin{array}{l}1742.7-2902 \\
\text { E }\end{array}$ & $\mathrm{x} 1^{\prime}$ & $\begin{array}{l}174242.1 \\
-290213 \\
359.9,-0.11\end{array}$ & [1403] & & $\begin{array}{l}0.2 \\
(0.9-4.0 \mathrm{keV}) \\
{[1403]}\end{array}$ & \\
\hline
\end{tabular}

Pos.: [1403]; in error box MXB 1743-29: [747].

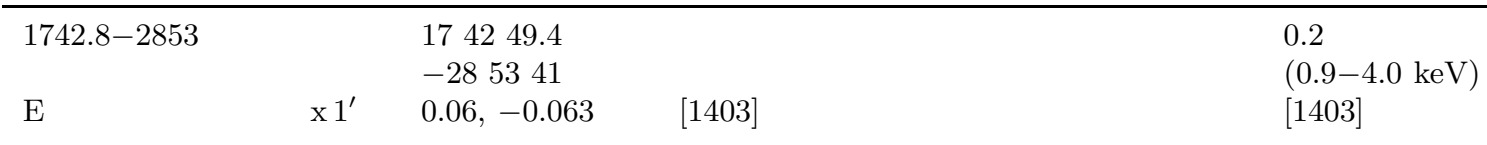

Pos.: [1403]; in error box MXB 1743-29: [747].

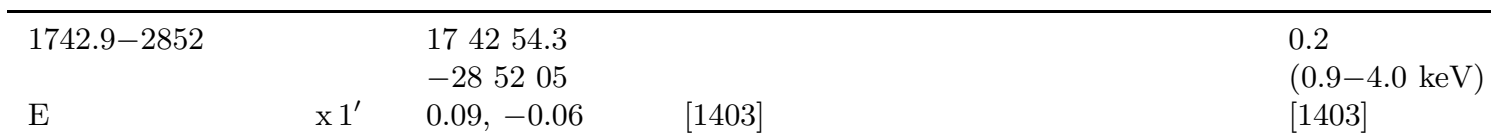

Pos.: [1403]; in error box MXB 1743-29: [747].

\begin{tabular}{|c|c|c|c|}
\hline $\begin{array}{l}1742-294 \\
\text { GC X-1 }\end{array}$ & B & $\begin{array}{l}174254.7 \\
-292958\end{array}$ & $60-180$ \\
\hline $\mathrm{A}, \mathrm{Gr}$ & $\mathrm{x} 1^{\prime}$ & $359.56,-0.39 \quad[634]$ & {$[1166,1233,1399]$} \\
\hline
\end{tabular}

Pos.: [526]; transient, X-ray bright since 1975: [112, 207, 262, 350, 666, 1064, 1166, 1168, 1237, 1403]; X-ray bursts (MXB 1742-29?): [747, 1008, 1233, 1240].

\begin{tabular}{llll}
\hline $1742.9-2849$ & 174259.2 & & 0.2 \\
& & -284957 & $(0.9-4.0 \mathrm{keV})$ \\
$\mathrm{E}$ & $\mathrm{x} 1^{\prime}$ & $0.13,-0.0626$ & {$[1403]$}
\end{tabular}

Pos.: [1403].

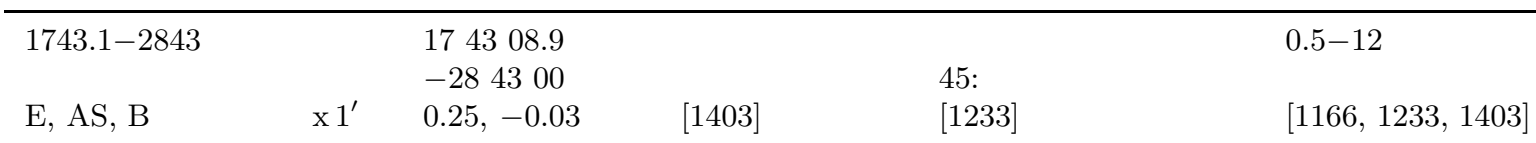

Pos.: [1403]; X-ray obs.: [666, 1237]; in error box MXB 1743-28: [747]; long-term X-ray obs.: [261].

$\left.\begin{array}{llll}\hline 1743.1-2852 & 174308.9 & & 0.2 \\ & & -285236 & (0.9-4.0 \mathrm{keV}) \\ \mathrm{E} & \mathrm{x} 1^{\prime} & 0.11,-0.11 & {[1403]}\end{array}\right]$

Pos.: [1403]; in error box MXB 1743-29: [747].

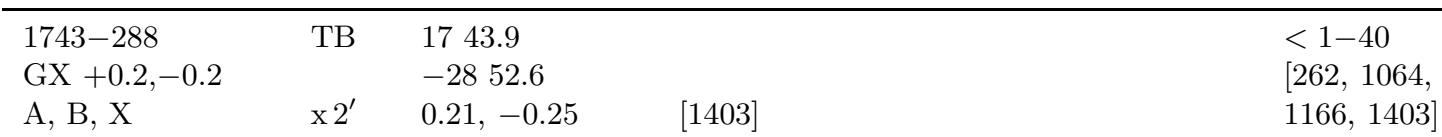

Pos.: [1064]; same source of SAX J1747.0-2853 (17 47 03.1, -28 52 30; J2000, 30"): [62, 136, 613]; X-ray history: [1064]; type-I bursts: [613, 1161]; probably same source as GC X-3: [262].

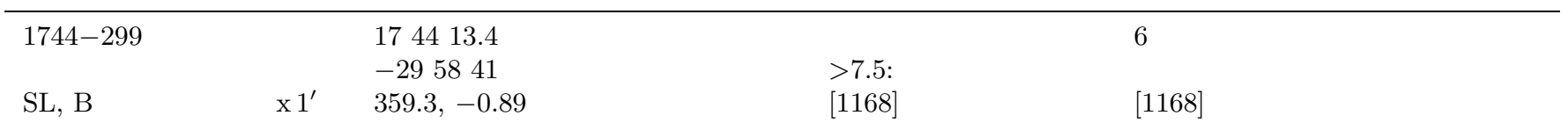

Pos.: [1168]; X-ray obs.: [1162]; northern component of two X-ray sources separated by 3': [1168]. 
Table 1. continued

\begin{tabular}{|c|c|c|c|c|c|c|}
\hline $\begin{array}{l}\text { Source } \\
\text { Name(s) } \\
\text { Spacecraft }\end{array}$ & $\begin{array}{l}\text { type } \\
\text { Pos. }\end{array}$ & $\begin{array}{l}\mathrm{RA} \\
\mathrm{DEC} \\
l^{\mathrm{II}}, b^{\mathrm{II}}\end{array}$ & $\begin{array}{l}\text { Opt. Ctp. } \\
{[\mathrm{FC}]}\end{array}$ & $\begin{array}{l}V, B-V, U-B \\
E_{B-V}\end{array}$ & $\begin{array}{l}F_{\mathrm{x}} \\
\mu \mathrm{Jy}\end{array}$ & $\begin{array}{l}P_{\text {orb }}(\mathrm{hr}) \\
P_{\text {pulse }}(\mathrm{s})\end{array}$ \\
\hline $\begin{array}{l}\text { J1744-28 } \\
\text { C, K, AS, X, Gr }\end{array}$ & $\begin{array}{l}\text { TBP } \\
\times 10^{\prime \prime}\end{array}$ & $\begin{array}{l}174433.1 \\
-284429 \\
0.04,+0.31\end{array}$ & [19] & & $\begin{array}{l}4700 \\
(8-20 \mathrm{keV}) \\
{[1120]}\end{array}$ & $\begin{array}{l}284.02 \\
0.467 \\
{[367]}\end{array}$ \\
\hline
\end{tabular}

Pos.: [19]; comparison with SMC X-1: [763]; comparison with the Rapid Burster (MXB 1730-335): [757]; the VLA radio source [388] slightly outside the ASCA error circle: [322]; hard X-ray spectroscopy: [1218]; dip after burst: [957, 1218]; phase lag during burst: [1218]; QPOs: [685, 1222, 1465]; magnetic field: [82, 264, 367, 1069]; major outbursts: [370, 693, 695]; small outbursts: [419, 627]; dip following some outbursts: [957, 1246]; type II burst: [142, 693, 757]; bursts during low persistent state likely type I: [82]; arrivel time of the pulse during bursts shifted.: [85, 689, 1208, 1218]; X-ray sp.: [957, 1246]; distance: [224, 957]; X-ray luminosity largely exceeding the Eddington limit of a NS: [419, 627]; iron feature in X-ray sp.: [957]; a stranger star: [181]; orbital parameters: [367]; very low mass function: [367]; system parameters: [1069]; near IR obs.: [19]; IR counterpart (?): [19]; evolutionary history: [284, 1069, 1225, 1359]; the burst source and the pulsar are one and the same source: [694]; the NS formation from collapse of white dwarf: [1359]; link between the LMXBs and recycled binary pulsars (?): [1069]; similar X-ray sp. of the bursts and persistent emission: [116, 1246]; spin-up of pulse period: [368]; opt. and near IR obs.: [224]; X-ray obs. of the second outburst and comparison with the first: [1450]; $589 \mathrm{~Hz}$ millisecond pulsation likely from MXB 1743-29 rather than this source: [1222].

\begin{tabular}{lllll}
\hline $1744-300$ & $\mathrm{~B}$ & 174413.6 & & 4 \\
$\mathrm{SL}, \mathrm{B}$ & $\mathrm{x} 1^{\prime}$ & $359.26,-0.9$ & {$[1168]$} & {$[1168]$}
\end{tabular}

Pos.: [1168]; X rays: [1162]; southern component of two X-ray sources separated by 3': [1168]; X-ray bursts: [1008, 1168, 1240].

\begin{tabular}{|c|c|c|c|c|c|}
\hline $1744-265$ & $\mathrm{BA}$ & 174448.9 & & & 400 \\
\hline GX3+1 & & -263249 & & 3.7 & \\
\hline $\begin{array}{l}\mathrm{U}, \mathrm{M}, \mathrm{A}, \mathrm{H}, \mathrm{S}, \mathrm{G}, \\
\mathrm{X}\end{array}$ & $\mathrm{x} 3^{\prime \prime}$ & $2.3,+0.8$ & {$[1072]$} & {$[527]$} & [1399] \\
\hline
\end{tabular}

Pos.: [527]; atoll source: [507]; X-ray bursts: [711, 799]; long-term X-ray var.: [13]; QPO: [751, 753, 802]; X-ray spectrum: [1380]; X-ray obs.: [1131]; IR obs.: [952]; radio obs.: [471].

\begin{tabular}{|c|c|c|c|c|}
\hline $\begin{array}{l}1744-361 \\
\text { A }\end{array}$ & $\begin{array}{l}\mathrm{T} \\
\mathrm{x} 40^{\prime \prime}\end{array}$ & $\begin{array}{l}174450.9 \\
-360654 \\
354.1, \quad-4.2\end{array}$ & $\begin{array}{l}<25-275 \\
{[103]}\end{array}$ & \\
\hline \multicolumn{5}{|c|}{ Pos.: [103]; X-ray transient 1976: $[144,230,294]$; uncertain if LMXB or HMXB. } \\
\hline $\begin{array}{l}\text { J1745.6-2901 } \\
\text { AS, B }\end{array}$ & $\begin{array}{l}\mathrm{TB} \\
\times 25^{\prime \prime}\end{array}$ & $\begin{array}{l}174536 \\
-290134 \\
359.9,-0.035\end{array}$ & $\begin{array}{l}0.4-2 \\
(3-10 \mathrm{keV}) \\
{[788]}\end{array}$ & $\begin{array}{l}8.4 \\
{[788]}\end{array}$ \\
\hline
\end{tabular}

Pos.: [1162]; type I X-ray burst and eclipse: [788]; a new LMXB: [669]; unlikely to associate with A1742-289: [669].

\begin{tabular}{|c|c|c|c|c|c|}
\hline $1745-248$ & TGB & $\begin{array}{l}174551 \\
-245245\end{array}$ & Ter 5 & 2.1 & $<0.1-110$ \\
\hline Ha, Exo & $\times 9^{\prime}$ & $3.8,+1.5$ & & {$[1405]$} & {$[525,1400]$} \\
\hline
\end{tabular}

Pos.: [1400]; X-ray bursts: [610, 797]; transient: [815]; X-ray obs.: [1369]; radio obs.: [390]; glob. cl. properties: [1405]; deep radio obs.: [391].

\begin{tabular}{|c|c|c|c|c|c|}
\hline $1745-203$ & $\mathrm{GT}$ & $\begin{array}{l}174555.0 \\
-202107\end{array}$ & NGC 6440 & 1.1 & $<0.1-180$ \\
\hline $\mathrm{U}, \mathrm{M}$ & $\mathrm{x} 1^{\prime}$ & $7.7,+3.8$ & {$[383,447,811]$} & {$[954,1405]$} & {$[345,383]$} \\
\hline
\end{tabular}

Pos.: [525]; transient (1971) probably in NGC 6440: [383, 811]; metal rich glob. cluster: [10]; optical study glob. cluster: [837, 1154]; JHK phm. obs. of NGC 6440: [705]; X rays: [345]; radio obs.: [390].

\begin{tabular}{|c|c|c|c|}
\hline $1746-331$ & $\mathrm{U}$ & $\begin{array}{l}1746 \quad 33.2 \\
-33 \quad 1103\end{array}$ & 27 \\
\hline $\mathrm{SL}, \mathrm{R}$ & $\mathrm{x} 35^{\prime \prime}$ & $356.9,-3.1 \quad[931]$ & {$[1168]$} \\
\hline
\end{tabular}

Pos.: [1168]; heavily reddened, probably identified with 1RXS J174948.4-331215: [931]; ultrasoft: [1413]; star A is a G-K type star: [931]. 
Table 1. continued

\begin{tabular}{|c|c|c|c|c|c|c|}
\hline $\begin{array}{l}\text { Source } \\
\text { Name(s) } \\
\text { Spacecraft }\end{array}$ & $\begin{array}{l}\text { type } \\
\text { Pos. }\end{array}$ & $\begin{array}{l}\mathrm{RA} \\
\mathrm{DEC} \\
l^{\mathrm{II}}, b^{\mathrm{II}}\end{array}$ & $\begin{array}{l}\text { Opt. Ctp. } \\
{[\mathrm{FC}]}\end{array}$ & $\begin{array}{l}V, B-V, U-B \\
E_{B-V}\end{array}$ & $\begin{array}{l}F_{\mathrm{x}} \\
\mu \mathrm{Jy}\end{array}$ & $\begin{array}{l}P_{\text {orb }}(\mathrm{hr}) \\
P_{\text {pulse }}(\mathrm{s})\end{array}$ \\
\hline $\begin{array}{l}1746.7-3224 \\
\mathrm{E}\end{array}$ & $\mathrm{x} 1^{\prime}$ & $\begin{array}{l}174647.3 \\
-322452 \\
357.5,-2.6\end{array}$ & & & $\begin{array}{l}0.1 \\
{[526,1168]}\end{array}$ & \\
\hline Pos.: [526]. & & & & & & \\
\hline $\begin{array}{l}1746-370 \\
\mathrm{U}, \mathrm{M}, \mathrm{A}, \mathrm{H}, \mathrm{S}, \mathrm{X}, \mathrm{B}\end{array}$ & $\begin{array}{l}\text { GBA } \\
\times 2^{\prime \prime}\end{array}$ & $\begin{array}{l}174648.5 \\
-370218 \\
353.5,-5.0\end{array}$ & $\begin{array}{l}\text { NGC } 6441 \\
{[475]}\end{array}$ & $\begin{array}{l}0.50 \\
{[1255]}\end{array}$ & $\begin{array}{l}32 \\
{[999,1366]}\end{array}$ & $\begin{array}{l}5.7 \\
{[1117]}\end{array}$ \\
\hline
\end{tabular}

Pos.: [475, 525]; metal rich glob. cluster: [10]; optical properties glob. cluster: [29, 528, 534]; cluster center: [525, 1154]; X-ray bursts: [758, 1255]; X-ray obs.: [1005, 1131]; X-ray dips (?): [999]; atoll source \& QPO: [644]; long-term X-ray behaviour: [1056]; radio obs.: [471]; opt. phm. of possible opt. cpt.: [311].

\begin{tabular}{|c|c|c|c|c|c|c|}
\hline $\begin{array}{l}1747-214 \\
\text { Exo }\end{array}$ & $\begin{array}{l}\mathrm{TB} \\
\times 7^{\prime \prime}\end{array}$ & \multicolumn{3}{|l|}{$\begin{array}{lll}17 & 47 & 25.7 \\
-21 & 2433\end{array}$} & \multicolumn{2}{|l|}{70} \\
\hline \multicolumn{7}{|c|}{ Pos.: [447]; X-ray bursts: [790]. } \\
\hline $1747-313$ & GT & $\begin{array}{l}17 \quad 47 \quad 31.2 \\
-31 \quad 16\end{array}$ & Ter 6 & 1.5 & $1.5-20$ & 12.36 \\
\hline $\mathrm{R}, \mathrm{Gr}, \mathrm{B}, \mathrm{X}$ & $\mathrm{x} 1^{\prime}$ & $358.6,-2.2$ & {$[182]$} & {$[1405]$} & {$[1019,1052]$} & {$[620]$} \\
\hline
\end{tabular}

Pos.: [1052]; ROSAT and Granat positions within $2^{\prime}$, likely same source: [1052, 1240]; X-ray observations: [1019]; IR \& opt. phm. of Terzan 6: [40, 353].

\begin{tabular}{llll}
\hline J1748-288 & T & 174805.06 & 640 \\
X, B, I & r $0.6^{\prime \prime}$ & -282825.8 & -0.21
\end{tabular}

Pos.: [558]; discovery: [1187]; radio counterpart: [558]; radio obs.: [359, 559, 1105]; radio jet: [1105]; X-ray light curve: [945, 1162]; QPO: [387]; X-ray obs.: [945, 1088]; BHC: [945, 1088]; X-ray sp. in high and low states: [1088].

\begin{tabular}{llllll}
\hline J1748.9-2021 & TGB & 174853.4 & ${ }^{*} \mathrm{~V} 2(?)$ & & 30 \\
& & -202143 & & 1.0 & \\
$\mathrm{~B}, \mathrm{X}, \mathrm{R}(?)$ & $\mathrm{x} 1^{\prime}$ & $7.73,+3.8$ & {$[1370]$} & {$[986]$} & {$[616]$}
\end{tabular}

Pos.: [558]; discovery in the globular cluster NGC 6440: [616]; distance of 8.5 kpc: [986]; broad-band sp.: [616]; X-ray light curve: [616]; type I burst: [616]; the same source of MX 1746-20 (?): [616]; not clear if associate with the ROSAT source: [616]; opt. obs.: [1370]; the possible opt. cpt. with $B$ of 22.7 mag: [1370]; uncertain if the same source of $1745-203$.

\begin{tabular}{llll}
\hline 1749-285 & $\mathrm{T}$ & 174906 & 60 \\
GX $+1.1,-1.0$ & & -282941 & \\
SL, A & $\times 5^{\prime}$ & $+1.1,-1.0$ & {$[1064]$} \\
Pos.: $[1064]$. & & & \\
\hline J1750.8-2900 & TB & 175024 & 130 \\
B, X & & -290218 & {$[948]$}
\end{tabular}

Pos.: [948]; discovery in Galactic center region: [60]; X-ray light curve: [948]; type I burst: [948]; burst sp.: [948]; distance of $7.0 \mathrm{kpc}:[948]$.

\begin{tabular}{llll}
\hline J1752.3-3138 & B & 175224 & 740 \\
& & -3137.7 & $(2-26 \mathrm{keV})$ \\
B & $\times 2.9^{\prime}$ & $358.4,-2.6$ & {$[60]$}
\end{tabular}

Pos.: [221]; discovery in Galactic center region: [221]; type I burst: [221]; burst sp.: [221]. 
Table 1. continued

\begin{tabular}{lllllll}
\hline $\begin{array}{l}\text { Source } \\
\text { Name(s) }\end{array}$ & type & RA & Opt. Ctp. & $V, B-V, U-B$ & $\begin{array}{l}F_{\mathrm{x}} \\
\mu J y\end{array}$ & $\begin{array}{l}P_{\text {orb }}(\mathrm{hr}) \\
P_{\text {pulse }}(\mathrm{s})\end{array}$ \\
Spacecraft & Pos. & $\begin{array}{l}\text { DEC } \\
l^{\mathrm{II}}, b^{\mathrm{II}}\end{array}$ & {$[\mathrm{FC}]$} & & \\
\hline $1755-338$ & DUT & 175521.5 & V4134 Sgr & $18.5,0.7,--$ & 100 & 4.46 \\
& & -334814 & & $0.5:$ & {$[1399]$} & {$[845,1417]$}
\end{tabular}

Pos.: [103]; ultrasoft X-ray sp.: [1411, 1420]; X-ray dips: [209, 210]; X-ray obs.: [1134]; a hard power-law tail: [994]; opt. sp.: [253]; opt. study: [845].

\begin{tabular}{llll}
\hline $\mathrm{J} 1755-324$ & $\mathrm{~T}$ & 175528.6 & 180 \\
& & -322839 & $(2-12 \mathrm{keV})$ \\
$\mathrm{X}, \mathrm{Gr}$ & $\mathrm{x} 1^{\prime}$ & $358.0,-3.6$ & {$[1078]$}
\end{tabular}

Pos.: [1078]; discovery in Galactic center region: [1078]; X-ray obs.: [431, 1086]; X-ray sp.: [431, 1086]; X-ray nova with a BH (?): [431, 1086]; X-ray light curve: [1086]; radio cpt. not found: [965].

\begin{tabular}{|c|c|c|c|c|c|}
\hline $1758-250$ & $\mathrm{Z}$ & 175803.1 & & & 1250 \\
\hline $\begin{array}{l}\text { GX } 5-1 \\
\mathrm{U}, \mathrm{M}, \mathrm{A}, \mathrm{H}, \mathrm{S}\end{array}$ & $x 3^{\prime \prime}$ & $\begin{array}{l}-250443 \\
5.1,-1.0\end{array}$ & {$[952,1072]$} & $\begin{array}{l}7.5: \\
{[527]}\end{array}$ & [1399] \\
\hline
\end{tabular}

Pos.: [527]; Z source: [507]; X-ray sp.: [712, 780, 1363, 1380]; QPO: [753, 755, 910, 960, 1327, 1329, 1331, 1333]; kHz QPOs: [1437]; phase-delay X-ray sp.: [1365]; X-ray obs.: [539, 1131]; long- and short-term X-ray var.: [14, 660]; long-term X-ray record: [1335]; no X-ray pulsations: [1449]; radio obs.: [106, 471]; X-ray/radio obs.: [1262]; IR cpt.: [643]; IR obs.: [952].

\begin{tabular}{lllll}
\hline $1758-258$ & $\mathrm{U}$ & 175806.7 & & 20 \\
$\mathrm{Gr}, \mathrm{X}, \mathrm{C}, \mathrm{R}, \mathrm{AS}$ & $\mathrm{r} 2^{\prime \prime}$ & $4.5,-1.4$ & {$[882,1165]$} & {$[1165]$}
\end{tabular}

Pos.: [1101]; BHC: [423, 883, 1238]; X-ray obs.: [887, 1190]; hard X-ray sp.: [1015, 1238]; long-term X-ray obs.: [423, 791]; soft X-ray excess: [883]; multiwvl. obs.: [769]; radio obs.: [1101]; two late-type stars in error box, search for the opt/IR cpt.: [833].

\begin{tabular}{lllll}
\hline $1758-205$ & $\mathrm{~A}$ & 175833.5 & & 700 \\
$\mathrm{GX} 9+1$ & & -203144 & $3.7:$ & \\
$\mathrm{U}, \mathrm{M}, \mathrm{A}, \mathrm{H}, \mathrm{S}$ & $\mathrm{x} 3^{\prime \prime}$ & $9.1,+1.2$ & {$[447,952,1072]$} & {$[527]$}
\end{tabular}

Pos.: [527]; atoll source: [507, 1327]; no X-ray pulsation: [1449]; QPO: [530]; X-ray spectrum: [1380, 1420]; X-ray obs.: [721, 1131]; IR obs.: [952]; radio obs.: [471].

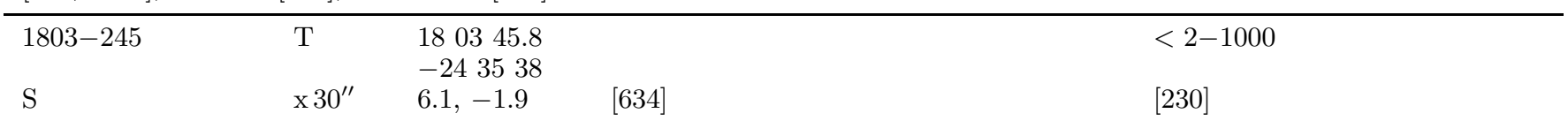

Pos.: [634]; transient 1976: [632, 634]; uncertain if LMXB or HMXB.

\begin{tabular}{llll}
\hline J1806-246 & TB(?)A & 180651 & 440 \\
$\mathrm{X}$ & $\mathrm{x} 1.5^{\prime}$ & -2435.1 & $(2-12 \mathrm{keV})$ \\
& & $6.14,-1.9$ & {$[826]$}
\end{tabular}

Pos.: [826]; discovery: [826]; possibly related to the burster SAX J1806.8-2435 \& 2S 1803-245: [826, 934]; likely radio cpt.: [557]; likely opt. $\operatorname{cpt}\left(V=22^{\mathrm{m}}\right)$ : [594]; X-ray light curve: [1087, 1428]; QPO: [1087, 1428]; X-ray sp.: [1428]; atoll source: [1428].

\begin{tabular}{|c|c|c|c|c|c|c|}
\hline $\begin{array}{l}\text { J1808.4-3658 } \\
\text { J1808-369 } \\
\text { B, X }\end{array}$ & $\begin{array}{l}\text { TPB } \\
\text { о } 0.8^{\prime \prime}\end{array}$ & $\begin{array}{l}1808 \quad 27.54 \\
-36 \quad 58 \quad 44.3 \\
355.4,-8.14\end{array}$ & {$[420]$} & $\begin{array}{l}16.6,--,-- \\
{[420,1097]}\end{array}$ & $\begin{array}{l}110 \\
{[420]}\end{array}$ & $\begin{array}{l}2.014167 \\
0.00249 \\
{[162,1425]}\end{array}$ \\
\hline
\end{tabular}

Pos.: [420]; discovery: [615, 827, 1425]; $V_{\text {quiesc }}<$ 20.5: [420]; a strange star: [765]; very low mass function: [162]; orbital parameters: [162]; opt. cpt.: [1097]; opt. photometry: [420]; opt. spectrum: [363]; X-ray very hard: [519, 520, 1426]; distance 4 kpc: [615]; bright type I X-ray burst: [615]; X-ray light curve: [615]; X-ray sp.: [431, 519, 615, 1426]; opt. in antiphase with X-ray modulation: [420]; opt. light curve: [420]; X-ray steep decline: [395]; X-ray sp. remainded constant over two orders of intensity decline: [395, 396]; opt. flux decline proceeding the steep X-ray decline: [420]; evolutionary scenario: [346]; magnetic field: [395, 1425]; transient radio emission, likely associated with an ejection of material from the system: [393]; rapid aperiodic X-ray variability: [1426, 1440]; near IR obs.: [1034, 1097]; phase lag: [270]; violent quasi-periodic X-ray flaring: [1339]. 
Table 1. continued

\begin{tabular}{|c|c|c|c|c|c|c|}
\hline $\begin{array}{l}\text { Source } \\
\text { Name(s) } \\
\text { Spacecraft }\end{array}$ & $\begin{array}{l}\text { type } \\
\text { Pos. }\end{array}$ & $\begin{array}{l}\mathrm{RA} \\
\mathrm{DEC} \\
l^{\mathrm{II}}, b^{\mathrm{II}}\end{array}$ & $\begin{array}{l}\text { Opt. Ctp. } \\
{[\mathrm{FC}]}\end{array}$ & $\begin{array}{l}V, B-V, U-B \\
E_{B-V}\end{array}$ & $\begin{array}{l}F_{\mathrm{x}} \\
\mu \mathrm{Jy}\end{array}$ & $\begin{array}{l}P_{\text {orb }}(\mathrm{hr}) \\
P_{\text {pulse }}(\mathrm{s})\end{array}$ \\
\hline $\begin{array}{l}\mathrm{J} 1810.8-2609 \\
\mathrm{~J} 1810.7-2609\end{array}$ & TB & $\begin{array}{l}181044.5 \\
-260901\end{array}$ & & $B=21.5, R=19.5$ & 16 & \\
\hline $\mathrm{B}, \mathrm{R}$ & $\mathrm{x} 10^{\prime \prime}$ & $5.20,-3.4$ & {$[461]$} & {$[461]$} & [1307] & \\
\hline
\end{tabular}

Pos.: [461]; discovery: [1307]; a strong type I burst: [949, 1307]; burst peak intensity 1.9 Crab: [949]; distance of 4.9 kpc: [949]; wideband X-ray sp.: [949]; opt./IR cpt.: [461].

\begin{tabular}{lllllll}
\hline $1811-171$ & AB & 181136.7 & IR star & $K=12$ & 350 & 592.8 \\
GX13+1 & & -171023 & & 5.7 & & \\
U, M, A, H, S, X & r, IR & $13.5,+0.1$ & {$[328,447,952]$} & {$[167]$} & {$[1399,1448]$} & {$[241]$}
\end{tabular}

Pos.: [406, 471]; atoll source: [507, 1327]; X-ray spectrum: [1380]; X-ray obs.: [1131]; X-ray bursts: [371, 850]; QPO: [574]; IR counterpart: [167]; opt. cpt.: [477]; IR obs.: [37, 39, 406, 952]; sp. type of K5III: [39]; X-ray/radio obs.: [404]; radio obs.: [471].

\begin{tabular}{llll}
\hline $1812-12$ & B & 1812.4 & 15 \\
M, A, H, B & x $12^{\prime}$ & $18.1,+2.3$ & {$[1399,1448]$}
\end{tabular}

Pos.: [937]; X-ray bursts: [222, 371, 937]; two Einstein IPC sources in error box: [349]; distance of 4 kpc: [222].

\begin{tabular}{lcclll}
\hline $1813-140$ & ZB & 181310.9 & NP Ser & $17.5,1.3,1.0$ & 700 \\
GX 17+2 & & -140315 & & $2.5:$ & \\
U, M, A, H, S, Exo & o & $16.4,+1.3$ & {$[328,447,952]$} & {$[807,1131,1254]$} & {$[1399]$}
\end{tabular}

Pos.: [103]; Z source: [507, 1327]; QPO: [724, 1031, 1216, 1430]; kHz QPOs: [1434]; X-ray spectrum: [713, 1380, 1420]; X-ray obs.: [1131]; no X-ray pulsations: [1449]; X-ray bursts: [648, 1254, 1271]; opt. cpt. (G star?): [288, 540, 564, 743, 1265]; opt. spectrum: [253]; IR obs.: [952]; IR candidate: [312]; NP Ser not the opt. cpt.: [312]; radio obs.: [471, 549]; radio/X-ray obs.: [1027, 1414]; no radio lobes: [1030].

\begin{tabular}{llllll}
\hline J1819.3-2525 & T & 181921.48 & V4641 Sgr & $8.8-13.5,--,--$ & $1-13000$ \\
J1819-254 & & -252536.0 & & 0.24 & $(2-12 \mathrm{keV})$ \\
B, X & o $0.7^{\prime \prime}$ & $6.75,-4.79$ & {$[436]$} & {$[1224,1388]$} & {$[1189]$}
\end{tabular}

Pos.: [1113]; discovery: [618]; X-ray light curve: [621]; strong Fe-K emission line: [621]; X-ray flare sp.: [621]; fast and large X-ray outbursts: [1189, 1429]; sp. become harder during decay: [1429]; considerbly softer in quiescent state: [1429]; BHC (?): [1429]; opt. outburst: [1224]; opt. sp.: [24, 174, 768, 985]; radio cpt.: [560]; radio obs.: [394, 560, 561, 563]; presence of radio ejecta: [561, 563]; distance: [563, 985]; possible orbital period of $2.866 \mathrm{~d}$ : [665, 985]; minimum mass of the compact star $2.55 M_{\odot}$ : [985]; sp. type of A2V: [985]; V4641 Sgr used to be misidentified as GM Sgr: [1113].

\begin{tabular}{lllllll}
\hline 1820-303 & GBA & 182027.8 & NGC 6624 & $B=18.7$ & 250 & 0.19 \\
& & -302317 & & 0.3 & & \\
U, M, A, H, S, X, B, R & x 3" & $2.8,-7.9$ & {$[475]$} & {$[674,1317]$} & {$[1366,1399]$} & {$[1213]$}
\end{tabular}

Pos.: [475, 525]; metal rich glob. cluster: [10]; structure glob. cluster: [528, 779]; cluster dynamics: [1065]; cluster HR diagram: [29]; atoll source: [507, 1327]; no X-ray pulsations: [1449]; X-ray spectrum: [780, 1380, 1420]; 176 day X-ray high-low period: [1060, 1182]; QPO: [319, 753, 1214, 1327, 1438]; kHz QPO: [1184]; X-ray obs.: [539, 650, 1131, 1212, 1337, 1376]; correlation between energy sp. states and fast time var.: [1469]; orbital period: [1178, 1261]; orbital period decrease: [1116,1261,1336]; Xray bursts: [217, 283, 472, 485, 1317, 1345]; radio obs.: [416, 471]; detected radio is from PSR 1820-30A: [81]; faint UV source: [1205]; $B$ and $V$ obs.: [1206]; reprocessing model: [11]; detected UV and opt. cpt.: [674].

\begin{tabular}{lllllll}
\hline $1822-371$ & $\mathrm{D}$ & 182222.7 & V691 CrA & $15.3-16.3,0.1,-0.9$ & $10-25$ & 5.57 \\
& & -370804 & & 0.15 & & \\
U, M, A, H, B & o & $356.9,-11.3$ & {$[463]$} & {$[840,841,842]$} & {$[872,1448]$} & {$[842,1415]$}
\end{tabular}

Pos.: [103]; orbital period change: [523]; X rays: [522, 1006]; opt. spectrum: [170, 250, 497, 844]; transient MeV range $\gamma$-ray source: [115]; near-IR sp.: [255]; UV obs.: [840, 841, 1066]; accretion disk corona: [522, 841, 1409]. 
Table 1. continued

\begin{tabular}{lllllll}
\hline $\begin{array}{l}\text { Source } \\
\text { Name(s) }\end{array}$ & type & RA & Opt. Ctp. & $V, B-V, U-B$ & $\begin{array}{l}F_{\mathrm{x}} \\
\mu \mathrm{Jy}\end{array}$ & $\begin{array}{l}P_{\text {orb }}(\mathrm{hr}) \\
E_{\text {pulse }}(\mathrm{s})\end{array}$ \\
Spacecraft & Pos. & $\begin{array}{l}\text { DEC } \\
l^{\mathrm{II}}, b^{\mathrm{II}}\end{array}$ & {$[\mathrm{FC}]$} & & $25-62$ \\
\hline $1822-000$ & & 182248.3 & star & $22,1:-,-$ & 1.3 & \\
& & -000229 & & {$[191,527]$}
\end{tabular}

Pos.: [527]; X-ray obs.: [536]; long-term X-ray obs.: [1059]; radio obs.: [471].

\begin{tabular}{|c|c|c|c|c|c|}
\hline $1826-238$ & TB & $\begin{array}{l}182624 \\
-23 \quad 4931\end{array}$ & & $19,0.4,-0.5$ & 30 \\
\hline $\mathrm{G}, \mathrm{B}, \mathrm{X}$ & $\mathrm{x} 0.9^{\prime}$ & $9.3,-6.0$ & [930] & {$[930]$} & {$[611,793]$} \\
\hline
\end{tabular}

Pos.: [611]; X-ray obs.: [617, 1139]; transient: [793]; orbital period $5.75 \mathrm{hr}$ or $2.1 \mathrm{hr}$ [581, 1306]; opt. phm.: [581]; X-ray bursts: [63, 617, 1306]; opt. burst: [581]; opt. cpt.(J2000 pos. of 1829 28.2, -23 4749.2 with 2" error): [930]; distance of 8 kpc: [617].

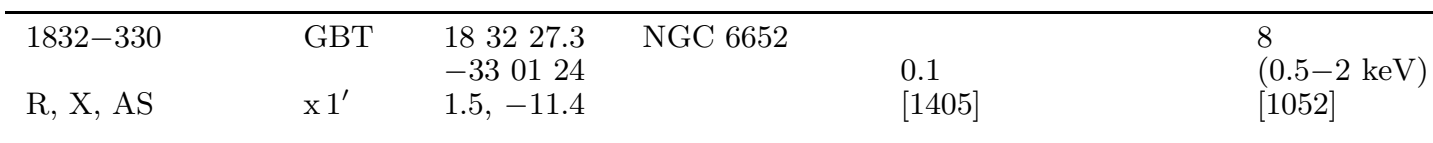

Pos.: [1052]; type-I burst: [614, 932]; X-ray obs.: [313, 932]; possible opt. cpt. with $m_{439}=20.2$ : [309]; moderately metal rich glob. cluster: [10]; opt. study gl. cl.: [517]; gl. distance of 9.3 kpc: [987]; opt. phm., orbital period of 0.73 hr: [313].

\begin{tabular}{|c|c|c|c|c|c|}
\hline $\begin{array}{l}1837+049 \\
\text { Ser X-1 } \\
\text { U, M, A, H, S }\end{array}$ & $\begin{array}{l}\mathrm{B} \\
\mathrm{O}\end{array}$ & $\begin{array}{l}183729.5 \\
+045920 \\
36.1,+4.8\end{array}$ & $\begin{array}{l}\text { MM Ser } \\
\text { *DS } \\
{[447,1285]}\end{array}$ & $\begin{array}{l}19.2 \mathrm{~B},--,-0.5 \\
0.04-\geq 0.4 \\
{[1142,1285]}\end{array}$ & $\begin{array}{l}225 \\
{[1059,1399]}\end{array}$ \\
\hline
\end{tabular}

Pos.: [1052]; X-ray obs.: [539, 650, 1131, 1376]; X-ray sp.: [780, 1380]; X-ray bursts: [761, 1252]; opt./X-ray bursts: [487]; opt. sp.: [253, 1142]; G5V star: [1142]; opt. phm.: [1383]; superpos. of two stars: [1383]; radio obs.: [471]; X-ray/radio obs.: [1313].

\begin{tabular}{|c|c|c|c|}
\hline $1846-031$ & $\mathrm{TU}$ & $\begin{array}{lll}18 & 46 & 39.8 \\
-03 & 07 & 12\end{array}$ & 300 \\
\hline Exo & $\mathrm{x} 15^{\prime \prime}$ & $29.9,-0.9$ & [1002] \\
\hline
\end{tabular}

Pos.: [1002]; alternative position (18 45 26.8, -03 25 39) also possible: [1002]; X-ray re-outburst: [1460]; ultra-soft X-ray spectrum with hard tail: [1002]; archival optical plate search: [1406].

\begin{tabular}{lllllll}
\hline $1850-087$ & GB & 185021.1 & NGC 6712 & $21.0,0.2,-0.9$ & 7 & $0.343 ?$ \\
& & -084604 & star S & 0.42 & & \\
U, A, H, S, B & o & $25.4,-4.3$ & {$[21,263,447]$} & {$[263,956]$} & {$[999,1366,1399]$} & {$[580]$}
\end{tabular}

Pos.: [263]; new opt. pos.: [415]; optical studies glob. cluster: [29, 263, 525, 528, 1154]; X-ray obs.: [229, 679]; radio source: [471, 735, 785]; opt. phm.: [4]; UV: [4, 580]; long-term X-ray obs.: [1059]; X-ray bursts: [571, 1248].

\begin{tabular}{llll}
\hline $\mathrm{J} 1856+053$ & $\mathrm{~T}$ & 185639 \\
& +0519.8 & 70 \\
$\mathrm{X}, \mathrm{C}$ & $\mathrm{x} 1.8^{\prime}$ & 38.27, \\
& & +1.26
\end{tabular}

Pos.: [824]; discovery: [824]; a LMXB: [48]; BHC (?): [48].

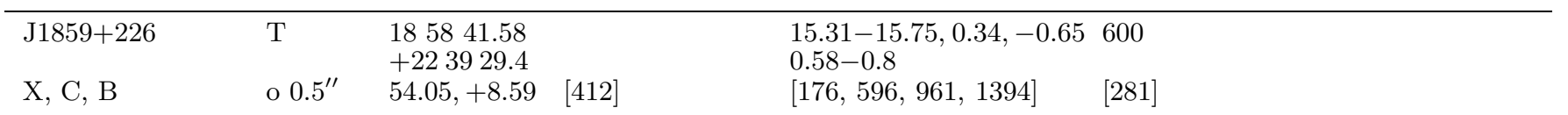

Pos.: [412]; discovery: [1446]; opt. cpt.: [412]; hard X-ray sp.: [281, 870]; QPO: [275, 281, 818]; kHz QPO: [275]; X-ray nova and BHC (?): [275, 281, 412, 818]; X-ray light curve: [275]; hard X-ray lag: [275]; opt. phm.: [412, 961]; opt. sp.: [412, 1394]; orbital period of 9.15 , but $14.79,6.60$ and $6.73 \mathrm{hr}$ still possible: [411, 1311]; IR obs.: [176]; UV sp.: [596]; radio cpt.: [1048].

\begin{tabular}{llllll}
\hline $1905+000$ & $\mathrm{~B}$ & 190553.4 & star & $20.5,0.5,-0.5$ & 10 \\
& & +000518 & & 0.5 & \\
$\mathrm{U}, \mathrm{A}, \mathrm{H}, \mathrm{S}$ & $\mathrm{x} 5^{\prime \prime}$ & $35.0,3.7$ & {$[194,447]$} & {$[194]$} & {$[188,1399]$}
\end{tabular}

Pos.: [194]; X rays: [188]; X-ray bursts: [188, 748, 851]; radio obs.: [471]. 
Table 1. continued

\begin{tabular}{lllllll}
\hline Source & type & RA & Opt. Ctp. & $V, B-V, U-B$ & $\begin{array}{l}F_{\mathrm{x}} \\
\mu \mathrm{Jy}\end{array}$ & $\begin{array}{l}P_{\text {orb }}(\mathrm{hr}) \\
P_{\text {pulse }}(\mathrm{s})\end{array}$ \\
$\begin{array}{l}\text { Name(s) } \\
\text { Spacecraft }\end{array}$ & Pos. & $\begin{array}{l}\text { DEC } \\
l^{\mathrm{II}}, b^{\mathrm{II}}\end{array}$ & {$[\mathrm{FC}]$} & & \\
\hline 1908+005 & TBA & 190842.8 & V1333 Aql & $14.8,0.6,-0.4$ & $<0.1-1300$ & 19.0 \\
Aql X-1 & & +003005 & & 0.4 & {$[103,1350]$} & {$[190]$}
\end{tabular}

Pos.: [103]; X-ray outbursts: [117, 169, 504, 657, 1059]; atoll source: [269, 1073]; QPO: [1467]; propeller effects: [1464]; opt. outbursts: [169, 783, 808, 1283]; opt. bursts: [1096]; long-term X-ray behaviour: [681, 1059]; magnetic field: [132]; V1333 Aql contained two stars: [129]; 309 d and 125 d periodicity: [903]; opt. sp.: [1142]; opt. phm. \& sp.: [409, 1146]; IR: [1146]; X-ray bursts: [277, 698]; fast X-ray decay: [134]; quiescent X-ray flux: [277, 1350]; 7.6 Hz pulsations in X-ray burst: [1124]; phm. of true opt. cpt. in quiescence and during outburst: [198]; secondary late K star: [198]; $V_{\text {quiesc }}=21.6$ : [198]; radio obs.: [554]; low-angle sys.: [1143, 1145].

\begin{tabular}{|c|c|c|c|c|c|}
\hline $1915+105$ & $\mathrm{~T}$ & $\begin{array}{l}191255 \\
+1052.8\end{array}$ & & $\begin{array}{l}I=23.4 \\
9.6\end{array}$ & 300 \\
\hline Gr, B, X, C, I, AS & $\times 3^{\prime}$ & $45.3,-0.9$ & {$[87]$} & {$[87]$} & {$[243]$} \\
\hline
\end{tabular}

Pos.: [243]; outburst history: [499]; soft $\gamma$-ray bursts: [898]; X-ray obs.: [460, 1011]; X-ray line: [691]; rapid X-ray var.: [1010]; X-ray var.: [460]; dips, regular and irregular bursts: [73, 460, 1012, 1256, 1453]; spin of the BH: [1463]; QPO: [178, 920, 1010]; phase lag: [265]; quasi-periodic var. in radio, IR and millimeter bands: [355, 358, 1047]; disk and jet interaction: [340, 505]; surrounding radio study: [1099]; IR phm.: [903]; faint IR flare: [342]; IR emission from radio emitting jets: [339]; radio/hard X-ray obs.: [385]; IR phm./radio obs.: [175, 354]; IR cpt.: [87]; IR sp.: [158, 341, 1445]; near IR jet: [1112]; opt. sp. \& phm.: [87]; superluminal radio ejection: [897]; radio obs.: [17, 360, 1047, 1098, 1102]; rapid radio flare: [358]; repeated relativistic ejections: [1100]; multiwvl obs.: [38, 905]; IR synchrotron emission: [358]; not surrounded by dense ISM: [1445]; a HMXB(?): [904]; model: [74, 268, 962, 1371]; distance of $12.5 \mathrm{kpc:} \mathrm{[897].}$

\begin{tabular}{|c|c|c|c|c|c|c|}
\hline $1916-053$ & $\mathrm{BD}$ & 191608.4 & V1405 Aql & $21.0,0.4,-0.5$ & 25 & 0.83 \\
\hline $\mathrm{U}, \mathrm{M}, \mathrm{A}, \mathrm{H}, \mathrm{S}$, & & -051941 & & 0.7 & & \\
\hline $\mathrm{R}, \mathrm{AS}, \mathrm{B}, \mathrm{X}, \mathrm{C}$ & o & $31.4,-8.5$ & $\begin{array}{l}{[447,} \\
1397]\end{array}$ & {$[476,1126]$} & {$[1179,1399]$} & {$[476,1126,1412]$} \\
\hline
\end{tabular}

Pos.: [447]; X-ray dips: [1179, 1181, 1303, 1397, 1412]; opt. phm.: [123]; opt. lt curve: [476, 1126]; X-ray and optical periods differ: [466, 1180]; X-ray bursts: [64, 1179, 1248, 1251]; QPO: [50]; X-ray obs.: [211, 683]; long-term X-ray obs.: [1059, 1182]; radio obs.: [471].

\begin{tabular}{llll}
\hline $1918+146$ & $\mathrm{~T}$ & 1918.0 & $<5-45$ \\
& & +1436.0 & {$[230]$}
\end{tabular}

Pos.: [1047]; soft X-ray spectrum: [230].

\begin{tabular}{|c|c|c|c|c|c|c|}
\hline $\begin{array}{l}1940-04 \\
\mathrm{Ha}\end{array}$ & $\begin{array}{l}\mathrm{B} \\
\times 1^{\circ}\end{array}$ & $\begin{array}{l}1940 \\
-4.0 \\
35.3,-13.1\end{array}$ & & & \multicolumn{2}{|l|}{$<50$} \\
\hline \multicolumn{7}{|c|}{ Pos.: [937]; X-ray bursts: [937]. } \\
\hline $\begin{array}{l}1957+115 \\
\text { U, M, A, H, S, } \\
\text { X, G, Exo, A S }\end{array}$ & $\mathrm{U}$ & $\begin{array}{l}195702.2 \\
+113416 \\
51.3,-9.3\end{array}$ & $\begin{array}{l}\text { V1408 Aql } \\
{[327,447]}\end{array}$ & $\begin{array}{l}18.7,0.3,-0.6 \\
0.4 \\
{[809]}\end{array}$ & $\begin{array}{l}30 \\
{[1399]}\end{array}$ & $\begin{array}{l}9.33 \\
{[1281]}\end{array}$ \\
\hline
\end{tabular}

Pos.: [809]; ultrasoft X-ray spectrum: [1411]; X-ray obs.: [1092, 1131]; long-term X-ray obs.: [1059]; likely to be a NS: [1456]; opt. phm.: [489]; opt. spectr: [253, 809, 1142].

\begin{tabular}{lllllll}
\hline $2000+251$ & TU & 200042.9 & QZ Vul & $18.9,1.3,0.0$ & $<0.5-11000$ & 8.26 \\
& & +250544 & ${ }^{*} \mathrm{~B}$ & 1.5 & {$[120,173$,} \\
$\mathrm{G}, \mathrm{R}$ & $\mathrm{o}$ & $63.4,-3.1$ & {$[120,447]$} & {$[90,1301]$} & {$[896,1139]$} & $189,191]$
\end{tabular}

Pos.: [968]; X-ray outburst: [1301]; X-ray obs. in quiescence: [1368]; soft X-ray component: [1301]; hard X-ray tail: [1229]; QPO: [1107]; BHC mass: [65, 127, 364, 496]; opt. sp.: [152, 364, 496]; secondary K3-6V star: [152, 496]; opt. obs. during outburst: [173, 189, 1017]; quiescence opt. phm.: [120, 192]; IR phm.: [127]; IR lt. curve: [65]; $R_{\text {quiesc }}=21.2$ : [120]; radio outburst: [550]. 
Table 1. continued

\begin{tabular}{lllllll}
\hline Source & type & RA & Opt. Ctp. & $V, B-V, U-B$ & $F_{\mathrm{x}}$ & $\begin{array}{l}P_{\text {orb }}(\mathrm{hr}) \\
P_{\text {pulse }}(\mathrm{s})\end{array}$ \\
Name(s) & & DEC & & & \\
Spacecraft & Pos. & $l^{\mathrm{II}}, b^{\mathrm{II}}$ & {$[\mathrm{FC}]$} & & 160 \\
\hline J2012+381 & $\mathrm{TU}$ & 201237.80 & ${ }^{*} \mathrm{~B}$ & $21.33,--,--$ & \\
& & +381101.1 & & $1.9-2.4$ & {$[1422]$} \\
$\mathrm{X}$, AS & $\mathrm{o}$ & $75.4,+2.22$ & {$[597]$} & {$[597]$} &
\end{tabular}

Pos.: [597]; discovery: [1079]; X-ray obs.: [823, 1079, 1422]; opt. cpt.: [597]; radio cpt.: [546, 547]; X-ray light curve: [597]; ultrasoft sp.: [1422]; BHC (?): [1422]; opt. obs.: [597]; radio obs.: [546, 547, 1046]; IR obs.: [128, 597].

\begin{tabular}{|c|c|c|c|c|c|c|c|}
\hline $2023+338$ & $\mathrm{TU}$ & $\begin{array}{l}202206.3 \\
+33 \quad 42 \quad 18\end{array}$ & V404 Cyg & $\begin{array}{l}12.7,1.5,0.3 \\
1.0:\end{array}$ & $0.4-20000$ & & 155.4 \\
\hline $\mathrm{G}, \mathrm{X}, \mathrm{K}$ & $\mathrm{o}$ & $73.2,-2.2$ & {$[331,1389]$} & {$[118,172,1390]$} & $\begin{array}{l}{[896,} \\
1234]\end{array}$ & 1139, & {$[148]$} \\
\hline
\end{tabular}

Pos.: [1389]; X-ray obs.: [1368]; X-ray outburst: [677, 1472]; hard X-ray tail: [1139, 1234]; rapid X-ray var.: [913, 974, 1139]; strong Fe emission line: [973]; soft X-ray transient: [1393]; opt. outburst: [147, 736, 1308, 1390]; previous outbursts 1938, 1956: [1093]; opt. phm.: [1018, 1308]; opt. sp.: [145, 147, 440]; opt. sp. \& phm.: [149]; the mass of BHC: [1118, 1139, 1141]; orbital period and mass function (BHC): [146, 148]; IR lt. curve: [1118, 1139]; IR sp.: [1141]; triple (?): [148, 1392, 1396]; high Li abundance in secondary: [836, 1396]; high-speed opt. phm.: [439]; radio obs.: [494, 543, 551]; quiescent ellipsoidal var.: [1392]; $V_{\text {quiesc }} \sim 19$ : [148]; UV obs.: [1392]; secondary K0 III-V star: [149]; evolutionary: [673].

\begin{tabular}{|c|c|c|c|c|c|c|c|}
\hline $\mathrm{J} 2123-058$ & TBA & 212314.54 & & $\begin{array}{l}16.8-19.24 \\
-0.88\end{array}$ & -0.02 & 110 & 5.96 \\
\hline $\mathrm{X}$ & o $1^{\prime \prime}$ & $\begin{array}{l}-054752.9 \\
58.10 \\
-29.9\end{array}$ & [1293] & $\begin{array}{l}0.1 \\
{[1290]}\end{array}$ & & {$[740]$} & {$[595,600,1293]$} \\
\hline
\end{tabular}

Pos.: [1293]; discovery at high galactic latitude: [740]; opt. bursts: [429, 1293]; type I X-ray bursts: [575, 1257, 1293]; opt. cpt.: [1290]; opt. phm.: [153, 600, 1203, 1293]; opt. sp.: [1290]; distance 10 kpc: [575, 1293]; kHz QPO: [575]; opt. lt. curve: [429, 1203]; an atoll source: [575]; X-ray lt. curve: [1293].

\begin{tabular}{lllllll}
\hline $2127+119$ & GB & 212733.3 & M15 & $15.8-16.4,-0.1,-1.2$ & 6 & 17.1 \\
& & +115651 & AC 211 & $<0.06$ & {$[521,604$,} \\
U, M, A, H, X & o & $65.0,-27.3$ & {$[22]$} & {$[23,83,953]$} & {$[1366,1399]$} & $605,951]$
\end{tabular}

Pos.: [414]; new opt. pos.: [415]; cluster center: [525, 1154]; metal poor globular cluster: [10]; cluster dynamics: [1036]; opt. studies glob. cl.: [278, 352, 528, 779, 1154]; opt. id.: [23, 171]; opt. spectrum: [950]; orbital decay: [579]; opt./UV sp.: [325]; X-ray bursts: [321, 1356]; period 37 d (?): [242]; UV obs.: [953]; radio cpt.: [785]; radio obs.: [471].

\begin{tabular}{lccclll}
\hline $2129+470$ & BT & 212936.2 & V1727 Cyg & $16.4-17.5,0.65,-0.3$ & 9 & 5.24 \\
& & +470408 & & $0.5:$ & & \\
U, M, A, H, R & o & $91.6,-3.0$ & {$[447,1284]$} & {$[864,1284]$} & {$[1399]$} & {$[864,865]$}
\end{tabular}

Pos.: [103]; X-ray obs. in quiesence: [400, 401]; X-ray orbital variations: [1314, 1409]; opt. phm./UV sp.: [308]; opt. phm. and sp.: [578, 1282, 1284]; X-ray bursts: [402]; low state: [407, 915, 1041]; no low-state ellipsoidal var.: [197, 248, 405, 659, 1286]; triple (?): [405]; accretion disk corona: [1409].

\begin{tabular}{lclllll}
\hline $2142+380$ & ZB & 214236.9 & V1341 Cyg & $14.7,0.5,-0.2$ & 450 & 236.2 \\
Cyg X-2 & & +380528 & & 0.45 & & \\
U, M, A, H, X, B, G & о & $87.3,-11.3$ & {$[418,447]$} & {$[199,783,866]$} & {$[1399]$} & {$[249]$}
\end{tabular}

Pos.: [103]; Z source: [507, 510]; X-ray spectrum: [201, 539, 650, 780, 1375, 1380, 1420]; no X-ray pulsations: [1449]; long-term X-ray record: [572, 1013, 1182, 1377]; 165Hz pulsation: [372]; QPO: [508, 907, 959, 1333]; kHz QPOs: [1435]; X-ray bursts: [649, 1171]; X-ray obs.: [538, 719, 1131, 1432]; burst-like events: [714]; low-energy X-ray line emission: [715]; Fe line: [1183]; 77.7 day modulation: [1431]; magnetic field: [132, 372]; multi-frequency campaign: [510, 552, 1355, 1378]; opt. spectrum: [154, 701]; companion F giant: [249]; near-IR spectrum.: [255]; opt. lt. curve: [249, 437, 982]; UV obs.: [199, 806, 866, 1378]; linear polarization of the UV/opt. cpt.: [684]; radio source: [542]; distance of $7.2 \mathrm{kpc}$ [ [982].

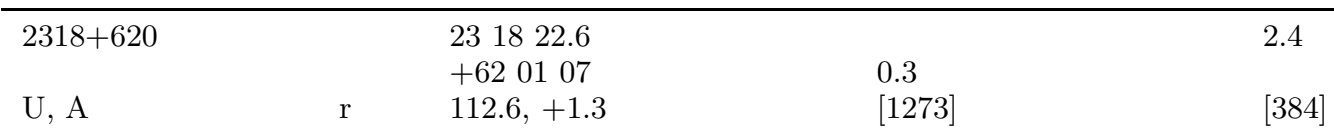

Pos.: [1273]; radio source with jet, identification with 4U2316+61 and 20th mag. star not certain: [1273]; prob. background galaxy: [362]. 
In Col. 6, the average X-ray flux, or the range of observed X-ray fluxes $(2-10 \mathrm{keV}$, unless otherwise indicated), is given, in units of

$$
\begin{aligned}
1 \mu \mathrm{Jy} & =10^{-29} \mathrm{erg} \mathrm{cm}^{-2} \mathrm{~s}^{-1} \mathrm{~Hz}^{-1} \\
& =2.410^{-12} \mathrm{erg} \mathrm{cm}^{-2} \mathrm{~s}^{-1} \mathrm{keV}^{-1} .
\end{aligned}
$$

The first line in Col. 7 gives the orbital period in hours. The second line contains for X-ray pulsars the pulse period, in seconds.

\section{Remarks on some individual objects}

We wish to emphasize here that some sources listed in this catalogue are still uncertain. They need to be regarded with caution in view of the further work necessary. Some sources are tentatively classified as low-mass $\mathrm{X}$-ray binaries due to their X-ray burst feature and/or the similarity of the X-ray properties to other identified systems. No counterpart at other bands has been found.

Finally, we would like to make some remarks on several individual sources. The three luminous supersoft X-ray sources, RX J0527.8-6954, 1E 0543.8-6823 (CAL 83) and 1E 0547.6-7109 (CAL 87), are excluded from the new catalogue. The now generally accepted model for the luminous SSSs is that, with few exceptions, these sources are accreting white dwarfs (WDs) in binaries, which are burning hydrogen in their envelopes in a steady or intermittent way (van den Heuvel et al. 1992; Kahabka \& van den Heuvel 1997).

There is a new class of pulsating objects with spin periods in the 6-12 s range, which are different from "normal" binary X-ray pulsars (Mereghetti \& Stella 1995). These objects are referred to as braking X-ray pulsars (BXP) (Mereghetti \& Stella 1995) or anomalous X-ray pulsars (van Paradijs et al. 1995). These sources include 4U $0142+61$, 1E 2259+586, 1E 1048.1-5937, RX J1838.4-0301 (but see Mereghetti et al. 1997; Song et al. 2000 for RX J1838.4-0301) and the recently discovered sources 1E 1841-045 (Vasisht \& Gotthelf 1997), 1RXS J170849.0-400910 (Sugizaki et al. 1997), AX J1845.0-0358 (Torii et al. 1998), and probably RX J0720.4-3125 (Haberl et al. 1997). 4U 0142+61 and $1 \mathrm{E} 2259+586$ have already been listed in the previous LMXB catalogue, while 1E 1048.1-5937 was listed in the HMXB catalogue. Although it has been suggested that AXPs may consist of neutron stars accreting from a verylow-mass companion (Mereghetti \& Stella 1995) or are isolated neutron stars accreting from circumstellar debris (e.g. Corbet et al. 1995; van Paradijs et al. 1995), recently, evidence has been mounting that they are isolated neutron stars with very high magnetic fields, i.e., magnetars related to the soft gamma-ray repeaters (SGRs) (Kouveliotou et al. 1998, 1999; Hurley et al. 1999), as has been suggested by Thompson \& Duncan (1996). In view of these developments we have not included the AXPs in this LMXB catalogue, but in an Appendix we present a table of the suspected AXPs.

Acknowledgements. We are grateful to Robert Fender and Michiel van der Klis for carefully reading the manuscript and for useful comments. QZL acknowledges the financial support from KC Wong fellowship of Chinese Academy of Sciences. This work is partially supported by the Netherlands Organization for Scientific Research (NWO) through Spinoza Grant $08-0$ to E. P. J. van den Heuvel and by the National Project for Fundamental Research by the Ministry of Science and Technology of China (973 Project) through Grant G1999075405.

Noted added in proof: Rudy Wijnands informed us that we had missed two new sources SAX J1753.5-2349 and SAX J1806.5-2215 (in't Zand et al. 1998, axrs. symp. 228), while the sources SAX J0840.7+2248 (IAUC 6892), SAX J1719.6-4254 (IAUC 7263), XTE J1739-285 (IAUC 7300), XTE J1743-363 (IAUC 7210), and SAX J1818.6-1703 (IAUC 6840) are also likely LMXBs.

\section{Appendix: Anomalous X-ray pulsars}

There is growing evidence that anomalous X-ray pulsars are magnetars, a type of objects with dipolar magnetic fields much stronger than the critical magnetic field. Their common properties are:

(1) their spin periods are distributed in a narrow range (5-12 s), contrary to those of high-mass X-ray binary pulsars, which cover a much wider range;

(2) they have very soft X-ray spectra with a photon index about 3 or larger;

(3) their X-ray luminosity is relatively low $\left(10^{35}\right.$ $10^{36} \mathrm{erg} \mathrm{s}^{-1}$ ), compared to that of HMXB pulsars;

(4) their X-ray flux shows little variability on timescales from months to years (they are not transient systems);

(5) they have a relatively stable spin period evolution, with long intervals of nearly constant spin-down;

(6) they are located in the galactic plane with the galactic latitude $|b| \leq 1^{\circ}$;

(7) their optical counterparts, it present, are very faint of implying that at least they cannot have a massive companion; and

(8) a few of them are possibly associated with supernova remnants (SNRs).

In this appendix we present a simple table of these anomalous X-ray pulsars (Table 2). Further identification is needed for some AXP candidates, although we have 
[45] Barret, D., et al. 1992, ApJ, 394, 615

[46] Barret, D., et al. 1993, A\&AS, 97, 241

[47] Barret, D., et al. 1995, A\&A, 296, 459

[48] Barret, D., et al. 1996, IAU Circ., 6519

[49] Barret, D., et al. 1996, ApJ, 473, 963

[50] Barret, D., et al. 1997, IAU Circ., 6793

[51] Barret, D., et al. 1998, A\&A, 329, 965

[52] Barret, D., et al. 1999, A\&A, 341, 789

[53] Barret, D., et al. 2000, ApJ, 533, 329

[54] Bartolini, C., et al. 1994, ApJS, 92, 455

[55] Barziv, O., et al. 1997, A\&A, 325, 1035

[56] Basinska, E. M., et al. 1984, ApJ, 281, 337

[57] Baykal, A., \& Swank, J. 1996, ApJ, 460, 470

[58] Baykal, A., et al. 1998, A\&A, 336, 173

[59] Bazzano, A., et al. 1992, ApJ, 385, L17

[60] Bazzano, A., et al. 1997, IAU Circ., 6597

[61] Bazzano, A., et al. 1997, IAU Circ., 6668

[62] Bazzano, A., et al. 1998, IAU Circ., 6873

[63] Becker, R. H., et al. 1976, IAU Circ., 2953

[64] Becker, R. H., et al. 1977, ApJ, 216, L101

[65] Beekman, G., et al. 1996, MNRAS, 281, L1

[66] Beekman, G., et al. 1997, MNRAS, 290, 303

[67] Belian, R. D., et al. 1972, ApJ, 171, L87

[68] Belian, R. D., et al. 1976, ApJ, 206, L135

[69] Belloni, T. 1994, IAU Circ., 6050.

[70] Belloni, T., \& Hasinger, G. 1990, A\&A, 230, 103

[71] Belloni, T., et al. 1991, A\&A, 245, L29

[72] Belloni, T., et al. 1993, A\&A, 271, 487

[73] Belloni, T., et al. 1997, ApJ, 479, L145

[74] Belloni, T., et al. 1997, ApJ, 488, L109

[75] Belloni, T., et al. 1997, A\&A, 322, 857

[76] Belloni, T., et al. 1999, ApJ, 519, L159

[77] Berger, M., \& van der Klis, M. 1998, A\&A, 340, 143

[78] Berger, M., et al. 1996, ApJ, 469, L13

[79] Beskin, G., et al. 1994, A\&A, 289, 141

[80] Bianchini, A., et al. 1997, A\&A, 321, 477

[81] Biggs, J. D., et al. 1994, MNRAS, 267, 125

[82] Bildsten, L., \& Brown, E. F. 1997, ApJ, 477, 897

[83] Bingham, E. A., et al. 1984, MNRAS, 209, 765

[84] Blair, W. P., et al. 1984, ApJ, 278, 270

[85] Blanco, P., et al. 1996, IAU Circ., 6321

[86] Bochkarev, N. G., et al. 1988, SvA Lett., 14, 421

[87] Boeer, M., et al. 1996, A\&A, 305, 835

[88] Bonnet-Bidaud, J. M., \& Mouchet, M. 1995, A\&A, 293, L69

[89] Bonnet-Bidaud, J. M., et al. 1989, A\&A, 213, 97

[90] Borisov, N. V. 1989, in Ref. [1421], 305

[91] Boroson, B., et al. 1996, ApJ, 473, 1079

[92] Boroson, B., et al. 1997, ApJ, 491, 903

[93] Borozdin, K. N., et al. 1994, IAU Circ., 6083

[94] Borozdin, K. N., et al. 1994, IAU Circ., 6088

[95] Borozdin, K. N., et al. 1998, Astron. Lett., 24, 435

[96] Borozdin, K. N., et al. 1999, ApJ, 517, 367

[97] Borozdin, K. N., et al. 2000, ApJ, 533, L131

[98] Borrel, V., et al. 1996, ApJ, 462, 754

[99] Bouchacourt, P., et al. 1984, ApJ, 285, L67

[100] Boynton, P. E., et al. 1973, ApJ, 186, 617

[101] Bradshaw, C. F., et al. 1997, ApJ, 481, 489

[102] Bradshaw, C. F., et al. 1999, ApJ, 512, L121

[103] Bradt, H. V., \& McClintock, J. E. 1983, ARA\&A, 21, 63

[104] Bradt, H. V., et al. 1975, ApJ, 197, 443

[105] Bradt, H. V., et al. 1977, Nature, 269, 496

[106] Braes, L. L. E., et al. 1972, Nature, 236, 392

[107] Brandt, S., et al. 1992, A\&A, 254, L39
[108] Brandt, S., et al. 1992, A\&A, 262, L15

[109] Brandt, S., et al. 1993, A\&AS, 97, 299

[110] Brandt, W. N., \& Schulz, N. S. 2000, ApJL, submitted

[111] Brandt, W. N., et al. 1996, MNRAS, 283, 1071

[112] Branduardi, G., et al. 1976, MNRAS, 175, 47p

[113] Branduardi-Raymont, G., et al. 1983, MNRAS, 205, 403

[114] Breedon, L. M., et al. 1986, MNRAS, 218, 487

[115] Briggs, M. S., et al. 1995, ApJ, 442, 638

[116] Briggs, M. S., et al. 1996, IAU Circ., 6290

[117] Buff, J., et al. 1977, ApJ, 212, 768

[118] Buie, M. W., \& Bond, H. E. 1989, IAU Circ., 4786

[119] Buxton, M., et al. 1998, IAU Circ., 6815

[120] Callanan, P. J., \& Charles, P. A. 1991, MNRAS, 249, 573

[121] Callanan, P. J., et al. 1990, A\&A, 240, 346

[122] Callanan, P. J., et al. 1992, MNRAS, 259, 395

[123] Callanan, P. J., et al. 1995, PASJ, 47, 153

[124] Callanan, P. J., et al. 1995, MNRAS, 273, 201

[125] Callanan, P. J., et al. 1995, ApJ, 441, 786

[126] Callanan, P. J., et al. 1996, ApJ, 461, 351

[127] Callanan, P. J., et al. 1996, ApJ, 470, L57

[128] Callanan, P., et al. 1998, IAU Circ., 6933

[129] Callanan, P. J., et al. 1999, IAU Circ., 7086

[130] Callanan, P. J., et al. 2000, A\&A, 355, 1049

[131] Camerron, R. A., et al. 1992, IAU Circ., 5587

[132] Campana, S. 2000, ApJ, 534, L79

[133] Campana, S., et al. 1997, A\&A, 324, 941

[134] Campana, S., et al. 1998, ApJ, 499, L65

[135] Campana, S., et al. 2000, A\&A, 358, 583

[136] Campana, S., et al. 2000, IAU Circ., 7401

[137] Campbell-Wilson, D., \& Hunstead, R. 1994, IAUC, 6052

[138] Campbell-Wilson, D., et al. 1998, IAU Circ., 7010

[139] Canizares, C. R., et al. 1975, ApJ, 197, 457

[140] Canizares, C. R., et al. 1979, ApJ, 234, 556

[141] Canizares, C. R., et al. 1980, ApJ, 236, L55

[142] Cannizzo, J. H. K. 1996, ApJ, 466, L31

[143] Carpenter, G. F., et al. 1975, IAU Circ., 2852

[144] Carpenter, G. F., et al. 1977, MNRAS, 179, 27p

[145] Casares, J., \& Charles, P. A. 1992, MNRAS, 255, 7

[146] Casares, J., \& Charles, P. A. 1994, MNRAS, 271, L5

[147] Casares, J., et al. 1991, MNRAS, 250, 712

[148] Casares, J., et al. 1992, Nature, 355, 614

[149] Casares, J., et al. 1993, MNRAS, 265, 834

[150] Casares, J., et al. 1995, MNRAS, 274, 565

[151] Casares, J., et al. 1995, MNRAS, 276, L35

[152] Casares, J., et al. 1995, MNRAS, 277, L45

[153] Casares, J., et al. 1998, IAU Circ., 6971

[154] Casares, J., et al. 1998, ApJ, 493, L39

[155] Castro-Tirado, A. J. 1995, IAU Circ., 6223

[156] Castro-Tirado, A. J., et al. 1992, IAU Circ., 5587

[157] Castro-Tirado, A. J., et al. 1993, A\&A, 276, L37

[158] Castro-Tirado, A. J., et al. 1996, ApJ, 461, L99

[159] Castro-Tirado, A. J., et al. 1997, A\&A, 322, 507

[160] Castro-Tirado, A. J., et al. 1998, IAU Circ., 7013

[161] Chakrabarty, D. 1998, ApJ, 492, 342

[162] Chakrabarty, D., \& Morgan, H. 1998, Nature, 394, 346

[163] Chakrabarty, D., \& Roche, P. 1997, ApJ, 489, 254

[164] Chakrabarty, D., et al. 1997, ApJ, 474, 414

[165] Chakrabarty, D., et al. 1997, ApJ, 481, L101

[166] Chakrabarty, D., et al. 1998, ApJ, 497, L39

[167] Charles, P. A., \& Naylor, T. 1992, MNRAS, 255, 6p

[168] Charles, P. A., et al. 1978, MNRAS, 183, 29p

[169] Charles, P. A., et al. 1980, ApJ, 237, 154

[170] Charles, P. A., et al. 1980, ApJ, 241, 1148

[171] Charles, P. A., et al. 1986, Nature, 323, 417 
[172] Charles, P. A., et al. 1989, in Ref. [1421], 103

[173] Charles, P. A., et al. 1991, MNRAS, 249, 567

[174] Charles, P. A., et al. 1999, IAU Circ., 7267

[175] Chaty, S., et al. 1996, A\&A, 310, 825

[176] Chaty, S., et al. 1999, IAU Circ., 7284

[177] Chaty, S., et al. 2000, IAU Circ., 7394

[178] Chen, X., et al. 1997, ApJ, 477, L41

[179] Cheng, F. H., et al. 1992, ApJ, 397, 664

[180] Cheng, F. H., et al. 1995, ApJ, 452, 825

[181] Cheng, K. S., et al. 1998, Science, 280, 407

[182] Cherepashchuk, A. M., et al. 1994, A\&A, 289, 419

[183] Chevalier, C. 1989, in Ref. [1421], 341

[184] Chevalier, C., \& Ilovaisky, S. A. 1974, A\&A, 35, 407

[185] Chevalier, C., \& Ilovaisky, S. A. 1981, A\&A, 94, L3

[186] Chevalier, C., \& Ilovaisky, S. A. 1982, A\&A, 112, 68

[187] Chevalier, C., \& Ilovaisky, S. A. 1987, A\&A, 172, 167

[188] Chevalier, C., \& Ilovaisky, S. A. 1990, A\&A, 228, 115

[189] Chevalier, C., \& Ilovaisky, S. A. 1990, A\&A, 238, 163

[190] Chevalier, C., \& Ilovaisky, S. A. 1991, A\&A, 251, L11

[191] Chevalier, C., \& Ilovaisky, S. A. 1992, private communication

[192] Chevalier, C., \& Ilovaisky, S. A. 1992, A\&A, 269, 301

[193] Chevalier, C., \& Ilovaisky, S. A. 1996, A\&A, 312, 105

[194] Chevalier, C., et al. 1985, A\&A, 147, L3

[195] Chevalier, C., et al. 1985, Sp. Sci. Rev., 40, 443

[196] Chevalier, C., et al. 1989, A\&A, 210, 114

[197] Chevalier, C., et al. 1989, A\&A, 217, 108

[198] Chevalier, C., et al. 1999, A\&A, 347, L51

[199] Chiappetti, L., et al. 1983, ApJ, 265, 354

[200] Chiappetti, L., et al. 1985, Sp. Sci. Rev., 40, 207

[201] Chiappetti, L., et al. 1990, ApJ, 361, 596

[202] Chodil, G., et al. 1968, ApJ, 152, L45

[203] Christian, C. A., \& Friel, E. D. 1992, AJ, 103, 142

[204] Churazov, E., et al. 1992, IAU Circ., 5623

[205] Churazov, E., et al. 1993, ApJ, 407, 752.

[206] Churazov, E., et al. 1994, IAU Circ., 6083

[207] Churazov, E., et al. 1995, ApJ, 443, 341

[208] Churazov, E., et al. 1996, ApJ, 464, L71

[209] Church, M. J., \& Batucinska-Church, M. 1993, MNRAS, 260,59

[210] Church, M. J., \& Batucinska-Church, M. 1997, A\&A, 317, L47

[211] Church, M. J., et al. 1998, A\&A, 338, 556

[212] Church, M. J., et al. 1998, ApJ, 504, 516

[213] Claret, A., et al. 1994, ApJ, 423, 436

[214] Clark, D. H., et al. 1975, Nature, 254, 674

[215] Clark, G. W. 1975, IAU Circ., 2843

[216] Clark, G. W., \& Li, F. 1977, IAU Circ., 3092

[217] Clark, G. W., et al. 1977 MNRAS, 179, 651

[218] Cocchi, M., et al. 1998, ApJ, 508, L163

[219] Cocchi, M., et al. 1999, A\&A, 346, L45

[220] Cocchi, M., et al. 1999, IAU Circ., 7247

[221] Cocchi, M., et al. 1999, IAU Circ., 7307

[222] Cocchi, M., et al. 2000, A\&A, 357, 527

[223] Coe, M. J., et al. 1994, MNRAS, 270, 178

[224] Cole, D. M., et al. 1997, ApJ, 480, 377

[225] Cominsky, L. R. 1980, Ph.D. Thesis M. I. T.

[226] Cominsky, L. R., et al. 1983, ApJ, 270, 226

[227] Cominsky, L., \& Wood, K. S. 1984, ApJ, 283, 765

[228] Cominsky, L., \& Wood, K. S. 1989, ApJ, 337, 485

[229] Cominsky, L., et al. 1977, ApJ, 211, L9

[230] Cominsky, L., et al. 1978, ApJ, 224, 46

[231] Conner, J. P., et al. 1969, ApJ, 157, L157

[232] Cook, L., et al. 2000, IAU Circ., 7397
[233] Cooke, B. A., et al. 1984, ApJ, 285, 258

[234] Cooke, B. A., \& Ponman, T. J. 1991, A\&A, 244, 358

[235] Corbel, S., et al. 2000, A\&A, 359, 251

[236] Corbet, R. H. D., et al. 1986, MNRAS, 222, 15p

[237] Corbet, R. H. D., et al. 1987, MNRAS, 227, 1055

[238] Corbet, R. H. D., et al. 1989, MNRAS, 239, 533

[239] Corbet, R. H. D., et al. 1994, ApJ, 436, L15

[240] Corbet, R. H. D., et al. 1995, ApJ, 443, 786

[241] Corbet, R., et al. 1996, IAU Circ., 6508

[242] Corbet, R., et al. 1997, IAU Circ., 6632

[243] Cordier, B., et al. 1992, Proc. Compton Symp., St Louis

[244] Cordier, B., et al. 1993, A\&A, 275, L1

[245] Cordier, B., et al. 1994, ApJS, 92, 401

[246] Cornelisse, R., et al. 2000, A\&A, 357, L21

[247] Courvoisier, T. J.-L., et al. 1986, ApJ, 309, 265

[248] Cowley, A. P., \& Schmitke, P. C. 1990, AJ, 99, 678

[249] Cowley, A. P., et al. 1979, ApJ, 231, 539

[250] Cowley, A. P., et al. 1982, ApJ, 255, 596

[251] Cowley, A. P., et al. 1982, ApJ, 256, 605

[252] Cowley, A. P., et al. 1987, AJ, 93, 195

[253] Cowley, A. P., et al. 1988, ApJ, 333, 906

[254] Cowley, A. P., et al. 1988, AJ, 95, 1231

[255] Cowley, A. P., et al. 1991, ApJ, 373, 228

[256] Crampton, D. 1974, ApJ, 187, 345

[257] Crampton, D., \& Hutchings, J. B. 1972, ApJ, 178, L65

[258] Crampton, D., \& Hutchings, J. B. 1974, ApJ, 191, 483

[259] Crampton, D., et al. 1986, ApJ, 306, 599

[260] Crampton, D., et al. 1990, ApJ, 355, 496

[261] Cremonesi, D. I., et al. 1999, A\&A, 345, 826

[262] Cruddace, R. G., et al. 1978, ApJ, 222, L95

[263] Cudworth, K. M. 1988, AJ, 96, 105

[264] Cui, W. 1997, ApJ, 482, L163

[265] Cui, W. 1999, ApJ, 524, L59

[266] Cui, W., et al. 1997, IAU Circ., 6604

[267] Cui, W., et al. 1997, ApJ, 487, L73

[268] Cui, W., et al. 1998, ApJ, 492, L53

[269] Cui, W., et al. 1998, ApJ, 502, L49

[270] Cui, W., et al. 1998, ApJ, 504, L27

[271] Cui, W., et al. 1999, IAU Circ., 7191

[272] Cui, W., et al. 1999, ApJ, 512, L43

[273] Cui, W., et al. 2000, ApJ, 529, 952

[274] Cui, W., et al. 2000, ApJ, 531, L45

[275] Cui, W., et al. 2000, ApJ, 535, L123

[276] Cutler, E. P., et al. 1986, ApJ, 300, 551

[277] Czerny, M., et al. 1987, ApJ, 312, 122

[278] Da Costa, G. S., \& Armandroff, T. E. 1990, AJ, 100, 162

[279] Dal Fiume, D., et al. 1990, Nuovo Cimento C, 13, 481

[280] Dal Fiume, D., et al. 1998, A\&A, 329, L41

[281] Dal Fiume, D., et al. 1999, IAU Circ., 7291

[282] Damen, E., et al. 1989, MNRAS, 237, 523

[283] Damen, E., et al. 1990, A\&A, 237, 103

[284] Daumerie, P., et al. 1996, Nature, 382, 141

[285] David, P., et al. 1997, A\&A, 322, 229

[286] David, P., et al. 1998, A\&A, 332, 165

[287] Davidsen, A., et al. 1974, ApJ, 193, L25

[288] Davidsen, A., et al. 1976, ApJ, 203, 448

[289] Davidsen, A., et al. 1977, ApJ, 211, 866

[290] Davies, R. D., et al. 1976, Nature, 261, 476

[291] Davies, S. R., \& Coe, M. J. 1991, MNRAS, 249, 313

[292] Davies, S. R., et al. 1990, MNRAS, 245, 268

[293] Davis, R. J., et al. 1975, Nature, 299, 659

[294] Davison, P. J. N., et al. 1976, IAU Circ., 2925

[295] Day, C. S. R., et al. 1988, MNRAS, 231, 69

[296] Deeter, J., et al. 1976, ApJ, 206, 861 
[297] Deeter, J. E., et al. 1981, ApJ, 247, 1003

[298] Deeter, J. E., et al. 1991, ApJ, 383, 324

[299] Deeter, J. E., et al. 1998, ApJ, 502, 802

[300] De Kool, M. 1988, ApJ, 334, 336

[301] Delgado, A. J., et al. 1983, A\&A, 127, L15

[302] Della Valle, M., \& Benetti, S. 1993, IAU Circ., 5890

[303] Della Valle, M., et al. 1991, A\&A, 247, L33

[304] Della Valle, M., et al. 1991, Nature, 353, 50

[305] Della Valle, M., et al. 1994, A\&A, 290, 803

[307] Della Valle, M., et al. 1997, A\&A, 318, 179

[307] Della Valle, M., et al. 1998, A\&A, 329, 606

[308] Deutsch, E. W., et al. 1996, ApJ, 471, 979

[309] Deutsch, E. W., et al. 1998, AJ, 116, 1301

[310] Deutsch, E. W., et al. 1998, PASP, 110, 912

[311] Deutsch, E. W., et al. 1998, ApJ, 493, 775

[312] Deutsch, E. M., et al. 1999, ApJ, 524, 406

[313] Deutsch, E. W., et al. 2000, ApJ, 530, L21

[314] Dhawan, V., et al. 2000, IAU Circ., 7395

[315] Dieters, S. W., et al. 2000, ApJ, 538, 307

[316] Dolan, J. F., \& Tapia, S. 1989, PASP, 101, 1135

[317] Dolan, J. F., et al. 1987, ApJ, 322, 324

[318] Dotani, T., et al. 1989, PASJ, 41, 427

[319] Dotani, T., et al. 1989, PASJ, 41, 577

[320] Dotani, T., et al. 1990, ApJ, 350, 395

[321] Dotani, T., et al. 1990, Nature, 347, 534

[322] Dotani, T., et al. 1996, IAU Circ., 6368

[323] Dower, R. G., et al. 1978, Nature, 273, 364

[324] Dower, R. G., et al. 1982, ApJ, 261, 228

[325] Downes, R. A., et al. 1996, PASP, 108, 688

[326] Doxsey, R. E., et al. 1973, ApJ, 182, L25

[327] Doxsey, R. E., et al. 1977, Nature, 269, 112

[328] Doxsey, R. E., et al. 1977, Nature, 270, 586

[329] Doxsey, R., et al. 1977, IAU Circ., 3113

[330] Doxsey, R., et al. 1979, ApJ, 228, L67

[331] Duerbeck, H. W. 1987, Sp. Sci. Rev., 45, 1

[332] Duerbeck, H. W., \& Walter, K. 1976, A\&A, 48, 141

[333] Duldig, M. L., et al. 1979, MNRAS, 187, 567

[334] Duncan, A. R., et al. 1993, MNRAS, 265, 157

[335] Dupree, A., et al. 1978, Nature, 275, 400

[336] Eachus, L. J., et al. 1976, ApJ, 203, L17

[337] Ebisawa, K., et al. 1994, PASJ, 46, 375

[338] Egonsson, J., \& Hakala, P. 1991, A\&A, 244, L41

[339] Eikenberry, S. S., \& Fazio, G. G. 1997, ApJ, 475, L53

[340] Eikenberry, S. S., et al. 1998, ApJ, 494, L61

[341] Eikenberry, S. S., et al. 1998, ApJ, 506, L31

[342] Eikenberry, S. S., et al. 2000, ApJ, 532, L33

[343] Elvis, M., et al. 1975, Nature, 257, 656

[344] Endo, T., et al. 2000, PASJ, 52, 223

[345] Ercan, N., et al. 1988, Ap. Lett. Comm., 26, 349

[346] Ergma, E., \& Antipova, J. 1999, A\&A, 343, L45

[347] Esin, A. A., et al. 2000, ApJ, 532, 1069

[348] Evans, W. D., et al. 1970, ApJ, 159, L57

[349] Exosat \& Einstein Data Bases

[350] Eyles, C. J., et al. 1975, Nature, 257, 291

[351] Fabbiano, G., et al. 1978, ApJ, 221, L49

[352] Fahlman, G. G., et al. 1985, ApJS, 58, 225

[353] Fahlman, G. G., et al. 1995, AJ, 110, 2189

[354] Fender, R. P., \& Pooley, G. G. 1998, MNRAS, 300, 573

[355] Fender, R. P., \& Pooley, G. G. 2000, MNRAS, 318, L1

[356] Fender, R. P., et al. 1997, IAU Circ., 6779

[357] Fender, R. P., et al. 1997, MNRAS, 286, L29

[358] Fender, R. P., et al. 1997, MNRAS, 290, L65

[359] Fender, R. P., et al. 1998, IAUC, 6937

[360] Fender, R. P., et al. 1999, MNRAS, 304, 865
[361] Fender, R. P., et al. 1999, ApJ, 519, L165

[362] Fender, R. P., 2000, private communication

[363] Filippenko, A. V., \& Leonard, D. C. 1998, IAU Circ., 6897

[364] Filippenko, A. V., et al. 1995, ApJ, 455, L139

[365] Filippenko, A. V., et al. 1995, ApJ, 455, 614

[366] Filippenko, A. V., et al. 1997, PASP, 109, 461

[367] Finger, M. H., et al. 1996, Nature, 381, 291

[368] Finger, M. H., et al. 1996, IAU Circ., 6286

[369] Fishman, G. J., et al. 1991, IAU Circ., 5395

[370] Fishman, G. J., et al. 1995, IAU Circ., 6272

[371] Fleischman, J. R. 1985, A\&A, 153, 106

[372] Focke, W. B. 1996, ApJ, 470, L127

[373] Fomalont, E. B., \& Geldzahler, B. J. 1991, ApJ, 383, 289

[374] Ford, E. 1997, ApJ, 475, L123

[375] Ford, E. 1999, ApJ, 512, L31

[376] Ford, E. C., \& van der Klis, M. 1998, ApJ, 506, L39

[377] Ford, E., et al. 1996, ApJ, 469, L37

[378] Ford, E., et al. 1997, ApJ, 486, L47

[379] Ford, E., et al. 1998, ApJ, 498, L41

[380] Ford, E. C., et al. 1998, ApJ, 508, L155

[381] Ford, E. C., et al. 1999, ApJ, 512, L31

[382] Forman, W., \& Jones, C. 1976, ApJ, 207, L177

[383] Forman, W., et al. 1976, ApJ, 207, L25

[384] Forman, W., et al. 1978, ApJS, 38, 357

[385] Foster, R. S. 1996, ApJ, 467, L81

[386] Foster, A. J., et al. 1986, MNRAS, 221, 409

[387] Fox, D., \& Lewin, W. 1998, IAU Circ., 6934

[388] Frail, D. A., et al. 1996, IAU Circ., 6307

[389] Francey, R. J. 1971, Nat. Phys. Sci., 229, 229

[390] Fruchter, A. S., \& Goss, W. M. 1990, ApJ, 365, L63

[391] Fruchter, A. S., \& Goss, W. M. 2000, ApJ, 536, 865

[392] Gaensler, B. M., et al. 1999, ApJ, 526, L37

[393] Gaensler, B. M., et al. 1999, ApJ, 522, L117

[394] Gaensler, B. M., et al. 1999, IAU Circ., 7256

[395] Galfanov, M., et al. 1998, A\&A, 338, L83

[396] Galfanov, M., et al. 1998, IAU Circ., 6897

[397] Gallagher, D., et al. 1995, ApJ, 439, 976

[398] Galloway, D. K., et al. 2000, MNRAS, 311, 755

[399] Gandolfi, G., et al. 1999, IAU Circ., 7174

[400] Garcia, M. R. 1994, ApJ, 435, 407

[401] Garcia, M. R., \& Callanan, P. J. 1999, AJ, 118, 1390

[402] Garcia, M. R., \& Grindlay, J. E. 1987, ApJ, 313, L59

[403] Garcia, M., et al. 1983, ApJ, 267, 291

[404] Garcia, M. R., et al. 1988, ApJ, 328, 552

[405] Garcia, M. R., et al. 1989, ApJ, 341, L75

[406] Garcia, M. R., et al. 1992, AJ, 103, 1325

[407] Garcia, M., et al. 1992, IAU Circ., 5578

[408] Garcia, M. R., et al. 1996, ApJ, 460, 932

[409] Garcia, M. R., et al. 1999, ApJ, 518, 422

[410] Garcia, M., et al. 2000, IAU Circ., 7392

[411] Garnavich, P. M., \& Quinn, J. 2000, IAU Circ., 7388

[412] Garnavich, P.M., et al. 1999, IAU Circ., 7276.

[413] Gaudenzi, S., \& Polcaro, V. F. 1999, A\&A, 347, 473

[414] Geffert, M., et al. 1989, A\&A, 209, 423

[415] Geffert, M., et al. 1994, A\&A, 282, 989

[416] Geldzahler, B. J. 1983, ApJ, 264, L49

[417] Gerend, D., \& Boynton, P. 1976, ApJ, 209, 562

[418] Giacconi, R., et al. 1967, ApJ, 148, L129

[419] Giles, A. B., et al. 1996, ApJ, 469, L25

[420] Giles, A. B., et al. 1999, MNRAS, 304, 47

[421] Giles, A. B., et al. 2000, ApJ, 529, 447

[422] Gilfanov, M., et al. 1991, SvA Lett., 17, 437

[423] Gilfanov, M., et al. 1993, ApJ, 418, 844 
[424] Glass, I. S. 1978, MNRAS, 183, 335

[425] Glass, I. S. 1979, MNRAS, 187, 807

[426] Glass, I. S. 1994, MNRAS, 268, 742

[427] Glass, I. S., \& Feast, M. W. 1973, Nat. Phys. Sci., 245, 39

[428] Gliozzi, M., et al. 1998, A\&A, 337, L39

[429] Gneiding, C. D., et al. 1999, A\&A, 352, 543

[430] Goldoni, P., et al. 1998, A\&A, 329, 186

[431] Goldoni, P., et al. 1999, ApJ, 511, 847

[432] Goldwurm, A., et al. 1992, ApJ, 389, L79

[433] Goldwurm, A., et al. 1992, IAU Circ., 5589

[434] Goldwurm, A., et al. 1996, A\&A, 310, 857

[435] Gonzalez-Riestra, R., et al. 1991, IAU Circ., 5174

[436] Goranskij, V. P. 1990, IBVS, 3464, 1

[437] Goranskij, V. P., \& Lyutyj, V. M. 1988, SvA, 32, 193

[438] Goss, W. M., \& Mebold, U. 1977, MNRAS, 181, 255

[439] Gotthelf, E., et al. 1991, ApJ, 374, 340

[440] Gotthelf, E., et al. 1992, AJ, 103, 219

[441] Gotthelf, E. V., et al. 1999, ApJ, 522, L49

[442] Gottlieb, E. W., et al. 1975, ApJ, 195, L33

[443] Gottwald, M., et al. 1986, ApJ, 308, 213

[444] Gottwald, M., et al. 1987, ApJ, 323, 575

[445] Gottwald, M., et al. 1987, MNRAS, 229, 395

[446] Gottwald, M., et al. 1989, ApJ, 339, 1044

[447] Gottwald, M., et al. 1991, A\&AS, 89, 367

[448] Grabelsky, D. A., et al. 1995, ApJ, 441, 800

[449] Gray, A. D., et al. 1991, Nature, 353, 237

[450] Grebenev, S., \& Sunyaev, R. 1991, IAU Circ., 5354

[451] Grebenev, S. A., et al. 1991, SvA Lett., 17, 413

[452] Grebenev, S. A., et al. 1992, SvA Lett., 18, 5

[453] Greenhill, J. G., et al. 1979, Nature, 279, 620

[454] Greenhill, J. G., et al. 1989, A\&A, 208, L1

[455] Greenhill, J. G., et al. 1995, MNRAS, 274, L59

[456] Greenhill, J. G., et al. 1999, PASA, 16, 240

[457] Gregory, P. C., \& Fahlmann, G. G. 1980, Nature, 287, 805

[458] Greiner, J., et al. 1995, A\&A, 297, L67

[459] Greiner, J., et al. 1996, A\&A, 314, L21

[460] Greiner, J., et al. 1996, ApJ, 473, L107

[461] Greiner, J., et al. 1999, MNRAS, 308, L17

[462] Griffiths, R. E., et al. 1978, ApJ, 221, L63

[463] Griffiths, R. E., et al. 1978, Nature, 276, 247

[464] Grindlay, J. E. 1979, ApJ, 232, L33

[465] Grindlay, J. E. 1981, in X-ray Astronomy with the Einstein Satellite, ed. R. Giacconi (Reidel), 79

[466] Grindlay, J. E. 1989, in Ref. [1421], 121

[467] Grindlay, J., \& Gursky, H. 1976, ApJ, 209, L61

[468] Grindlay, J. E., \& Gursky, H. 1977, ApJ, 218, L117

[469] Grindlay, J. E., \& Hertz, P. 1981, ApJ, 247, L17

[470] Grindlay, J. E., \& Liller, W. 1978, ApJ, 220, L127

[471] Grindlay, J. E., \& Seaquist, E. R. 1986, ApJ, 310, 172

[472] Grindlay, J., et al. 1976, ApJ, 205, L127

[473] Grindlay, J. E., et al. 1978, Nature, 274, 567

[474] Grindlay, J. E., et al. 1980, ApJ, 240, L121

[475] Grindlay, J. E., et al. 1984, ApJ, 282, L13

[476] Grindlay, J. E., et al. 1988, ApJ, 334, L25

[477] Groot, P. J., et al. 1996, IAU Colloq., 158, 367

[478] Grove, J. E., et al. 1998, ApJ, 500, 899

[479] Grove, J. E., et al. 1998, ApJ, 502, L45

[480] Guamazzi, M., et al. 1998, A\&A, 339, 802

[481] Guainazzi, M., et al. 1999, A\&A, 349, 819

[482] Gursky, H., et al. 1978, ApJ, 223, 973

[483] Gursky, H., et al. 1980, ApJ, 237, 163

[484] Haberl, F., \& Pietsch, W. 1999, A\&A, 344, 521
[485] Haberl, F., et al. 1987, ApJ, 314, 266

[486] Haberl, F., et al. 1997, A\&A, 326, 662

[487] Hackwell, J. A., et al. 1979, ApJ, 233, L115

[488] Hakala, P. J., et al. 1998, A\&A, 333, 540

[489] Hakala, P. J., et al. 1999, MNRAS, 306, 701

[490] Haller, J. W., \& Melia, F. 1994, ApJ, 423, L109

[491] Hameury, J.-M., et al. 1997, ApJ, 489, 234

[492] Hammerschlag-Hensberge, G., et al. 1982, ApJ, 254, L1

[493] Han, X., \& Hjellming, R. M. 1992, IAU Circ., 5593

[494] Han, X., \& Hjellming, R. M. 1992, ApJ, 400, 304

[495] Hannikainen, D. C., et al. 1998, A\&A, 337, 460

[496] Harlaftis, E. T., et al. 1996, PASP, 108, 762

[497] Harlaftis, E. T., et al. 1997, MNRAS, 285, 673

[498] Harmon, B. A., et al. 1992, IAU Circ., 5504

[499] Harmon, B. A., et al. 1992, IAU Circ., 5619

[500] Harmon, B. A., et al. 1993, IAU Circ., 5874

[501] Harmon, B. A., et al. 1993, IAU Circ., 5900

[502] Harmon, B. A., et al. 1994, ApJ, 425, L17

[503] Harmon, B. A., et al. 1995, Nature, 374, 703

[504] Harmon, B. A., et al. 1996, A\&AS, 120, 197

[505] Harmon, B. A., et al. 1997, ApJ, 477, L85

[506] Harries, J. R., et al. 1967, Nature, 215, 38

[507] Hasinger, G., \& van der Klis, M. 1989, A\&A, 225, 79

[508] Hasinger, G., et al. 1986, Nature, 319, 469

[509] Hasinger, G., et al. 1989, ApJ, 337, 843

[510] Hasinger, G., et al. 1990, A\&A, 235, 131

[511] Hasinger, G., et al. 1990, IAU Circ., 5142

[512] Haswell, C. A., \& Shafter, A. W. 1990, ApJ, 359, L47

[513] Haswell, C. A., et al. 1993, ApJ, 411, 802

[514] Haswell, C. A., et al. 2000, IAU Circ., 7407

[515] Haswell, C. A., et al. 2000, IAU Circ., 7427

[516] Haynes, R. F., et al. 1978, MNRAS, 185, 661

[517] Hazen, M. L. 1989, AJ, 97, 771

[518] Heindl, W. A. 1994, ApJ, 430, 829

[519] Heindl, W., \& Smith, D. A. 1998, ApJ, 506, L35

[520] Heindl, W., et al. 1998, IAU Circ., 6878

[521] Hellier, C. 1994, MNRAS, 271, L21

[522] Hellier, C., \& Mason, K. O. 1989, MNRAS, 239, 715

[523] Hellier, C., et al. 1990, MNRAS, 244, 39p

[524] Hertz, P. 1987, ApJ, 315, L119

[525] Hertz, P., \& Grindlay, J. E. 1983, ApJ, 275, 105

[526] Hertz, P., \& Grindlay, J. E. 1984, ApJ, 278, 137

[527] Hertz, P., \& Grindlay, J. E. 1984, ApJ, 282, 118

[528] Hertz, P., \& Grindlay, J. E. 1985, ApJ, 298, 95

[529] Hertz, P., \& Wood, K. S. 1988, ApJ, 331, 764

[530] Hertz, P., et al. 1990, ApJ, 354, 267

[531] Hertz, P., et al. 1992, ApJ, 396, 201

[532] Hertz, P., et al. 1995, ApJ, 438, 385

[533] Hertz, P., et al. 1997, ApJ, 486, 1000

[534] Hesser, J., et al. 1976, ApJ, 203, 97

[535] Heyal, J. S., \& Hernquist, L. 1998, MNRAS, 297, L69

[536] Hill, R., et al. 1974, ApJ, 189, L69

[537] Hiltner, W. A., \& Mook, D. E. 1970, ARA\&A, 8, 139

[538] Hirano, T., et al. 1984, PASJ, 36, 769

[539] Hirano, T., et al. 1987, PASJ, 39, 619

[540] Hjellming, R. M. 1978, ApJ, 221, 225

[541] Hjellming, R. M. 1979, IAU Circ., 3369

[542] Hjellming, R. M., \& Blankenship, L. C. 1973, Nat. Ph. Sci., 243, 81

[543] Hjellming, R. M., \& Han, X. H. 1989, IAU Circ., 4879

[544] Hjellming, R. M., \& Rupen, M. P. 1995, Nature, 375, 464

[545] Hjellming, R. M., \& Rupen, M. P. 1997, IAU Circ., 6547

[546] Hjellming, R. M., \& Rupen, M. P. 1998, IAU Circ., 6924

[547] Hjellming, R. M., \& Rupen, M. P. 1998, IAU Circ., 6932 
[548] Hjellming, R. M., \& Wade, C. M. 1971, ApJ, 164, L1

[549] Hjellming, R. M., \& Wade, C. M. 1971, ApJ, 168, L21

[550] Hjellming, R. M., et al. 1988, ApJ, 335, L75

[551] Hjellming, R. M., et al. 1989, IAU Circ., 4790

[552] Hjellming, R. M., et al. 1990, A\&A, 235, 147

[553] Hjellming, R. M., et al. 1990, ApJ., 365, 681

[554] Hjellming, R. M., et al. 1990, IAU Circ., 5112

[555] Hjellming, R. M., et al. 1996, ApJ, 470, L105

[556] Hjellming, R. M., et al. 1996, IAU Circ., 6383

[557] Hjellming, R. M., et al. 1998, IAU Circ., 6900

[558] Hjellming, R. M., et al. 1998, IAU Circ., 6934

[559] Hjellming, R. M., et al. 1998, IAU Circ., 6937

[560] Hjellming, R. M., et al. 1999, IAU Circ., 7254

[561] Hjellming, R. M., et al. 1999, IAU Circ., 7265

[562] Hjellming, R. M., et al. 1999, ApJ, 514, 383

[563] Hjellming, R. M., et al. 1999, ApJ, L submitted

[564] Hoag, A., \& Weisberg, J. M. 1976, ApJ, 209, 908

[565] Hoffman, J. A., et al. 1976, ApJ, 210, L13

[566] Hoffman, J. A., et al. 1977, MNRAS, 179, 57p

[567] Hoffman, J. A., et al. 1977, ApJ, 217, L23

[568] Hoffman, J. A., et al. 1978, Nature, 271, 630

[569] Hoffman, J. A., et al. 1978, ApJ, 221, L57

[570] Hoffman, J. A., et al. 1979, ApJ, 233, L51

[571] Hoffman, J. A., et al. 1980, ApJ, 240, L27

[572] Holt, S. S., et al. 1979, ApJ, 233, 344

[573] Homan, J., \& van der Klis, M. 2000, ApJ, 539, 847

[574] Homan, J., et al. 1998, ApJ, 499, L41

[575] Homan, J., et al. 1999, ApJ, 513, L119

[576] Homan, J., et al. 1999, IAUC, 7121; 2000, ApJ, submitted

[577] Homan, J., et al. 1999, ApJ, 516, L91

[578] Home, K., et al. 1986, MNRAS, 218, 63

[579] Homer, L., \& Charles, P. A. 1998, NewA, 3, 435

[580] Homer, L., et al. 1996, MNRAS, 282, L37

[581] Homer, L., et al. 1998, MNRAS, 298, 497

[582] Honey, W. B., et al. 1988 IAU Circ., 4532

[583] Horne, K., et al. 1996, IAU Circ., 6406

[584] Howarth, I. D., \& Wilson, B. 1983, MNRAS, 202347

[585] Howarth, I. D., \& Wilson, B. 1983, MNRAS, 204, 1091

[586] Hudec, R., \& Wenzel, W. 1986, A\&A, 158, 396

[587] Hughes, V. A., et al. 1981, ApJ, 246, L127

[588] Hurley, K., et al. 1999, ApJ, 510, L111

[589] Hutchings, J. B., et al. 1981, IAU Circ., 3585

[590] Hutchings, J. B., et al. 1985, ApJ, 292, 670

[591] Hynes, R. I., \& Haswell, C.A. 1999, ApJ, 513, 477

[592] Hynes, R. I., et al. 1998, MNRAS, 299, L37

[593] Hynes, R. I., et al. 1998, MNRAS, 300, 64

[594] Hynes, R. I., et al. 1998, IAU Circ., 6905

[595] Hynes, R. I., et al. 1998, IAU Circ., 6976

[596] Hynes, R. I., et al. 1999, IAU Circ., 7294

[597] Hynes, R. I., et al. 1999, MNRAS, 305, L49

[598] Hynes, R. I., et al. 2000, ApJ, 539, L37

[599] Ilovaisky, S. A., \& Chevalier, C. 1981, IAU Circ., 3586

[600] Ilovaisky, S. A., \& Chevalier, C. 1998, IAU Circ., 6975

[601] Ilovaisky, S. A., et al. 1978, A\&A, 70, L19

[602] Ilovaisky, S. A., et al. 1980, MNRAS, 191, 81

[603] Ilovaisky, S. A., et al. 1986, A\&A, 164, 67

[604] Ilovaisky, S. A., et al. 1987, A\&A, 179, L1

[605] Ilovaisky, S. A., et al. 1993, A\&A, 270, 139

[606] Imamura, J. N., et al. 1987, ApJ, 314, L11

[607] Imamura, J. N., et al. 1990, ApJ, 365, 312

[608] Inoue, H., et al. 1981, ApJ, 250, L71

[609] Inoue, H., et al. 1984, PASJ, 36, 831

[610] Inoue, H., et al. 1984, PASJ, 36, 855

[611] in't Zand, J. J. M., et al. 1989, in Ref. [1421], 693
[612] in't Zand, J. J. M., et al. 1990, Adv. Space Res. 11(8), 187

[613] in't Zand, J., et al. 1998, IAU Circ., 6846

[614] in't Zand, J. J. M., et al. 1998, A\&A, 329, L37

[615] in't Zand, J. J. M., et al. 1998, A\&A, 331, L25

[616] in't Zand, J. J. M., et al. 1999, A\&A, 345, 100

[617] in't Zand, J. J. M., et al. 1999, A\&A, 347, 891

[618] in't Zand, J. J. M., et al. 1999, IAU Circ., 7119

[619] in't Zand, J. J. M., et al. 1999, IAU Circ., 7243

[620] in't Zand, J. J. M., et al. 2000, A\&A, 355, 145

[621] in't Zand, J. J. M., et al. 2000, A\&A, 357, 520

[622] Israel, G. L. 1994, ApJ, 433, L25

[623] Israel, G. L. 1999, A\&A, 346, 929

[624] Iwasawa, K., et al. 1992, PASJ, 44, 9

[625] Jablonski, F., \& Pereira, M. G. 1997, MNRAS, 289, L17

[626] Jablonski, F., et al. 1997, ApJ, 482, L171

[627] Jahoda, K., et al. 1996, IAU Circ., 6414

[628] Jain, A., et al. 1984, A\&A, 140, 179

[629] Jain, R. K., \& Bailyn, C. D. 2000, IAU Circ., 7400

[630] Jain, R. K., et al. 1999, ApJ, 517, L131

[631] Jain, R. K., et al. 1999, IAU Circ., 7187

[632] Jernigan, G. 1976, IAU Circ., 2957

[633] Jernigan, J. G., et al. 1977, Nature, 270, 321

[634] Jernigan, J. G., et al. 1978, Nature, 272, 701

[635] Johnston, H. M., et al. 1989, ApJ, 345, 492

[636] Johnston, H. M., et al. 1999, MNRAS, 308, 415

[637] Johnston, M. D., et al. 1979, ApJ, 233, 514

[638] Jones, C., et al. 1973, ApJ, 182, L109

[639] Jones, C., et al. 1976, ApJ, 210, L9

[640] Jones, M. H., \& Watson, M. G. 1989, in Ref. [1421], 439

[641] Jonker, P. G., et al. 1998, ApJ, 499, L191

[642] Jonker, P. G., et al. 1999, ApJ, 511, L41

[643] Jonker, P. G., et al. 2000, MNRAS, 315, L57

[644] Jonker, P. G., et al. 2000, ApJ, 531, 453

[645] Jonker, P. G., et al. 2000, ApJ, 537, 374

[646] Jung, G. V., et al. 1995, A\&A, 295, L23

[647] Kahabka, P., \& van den Heuvel, E. P. J. 1997, ARA\&A, 35,69

[648] Kahn, S. M., \& Grindlay, J. E. 1984, ApJ, 281, 826

[649] Kahn, S. M., \& Grindlay, J. E. 1984, ApJ, 283, 286

[650] Kallman, T. R., et al. 1989, ApJ, 345, 498

[651] Kallman, T. R., et al. 1991, ApJ, 370, 717

[652] Kallman, T., et al. 1998, ApJ, 502, 441

[653] Kaluzienski, L. J., \& Holt, S. S. 1977, IAUC, 3099 \& 3106

[654] Kaluzienski, L. J., et al. 1975, ApJ, 201, L121

[655] Kaluzienski, L. J., et al. 1976, ApJ, 208, L71

[656] Kaluzienski, L. J., et al. 1976, IAU Circ., 2935

[657] Kaluzienski, L. J., et al. 1977, Nature, 265, 606

[658] Kaluzienski, L. J., et al. 1980, ApJ, 241, 779

[659] Kaluzny, J. 1988, Acta Astron., 38, 207

[660] Kamado, Y., et al. 1997, PASJ, 49, 589

[661] Kaminker, A. D., et al. 1989, A\&A, 220, 117

[662] Kaptein, R. G., et al. 2000, A\&A, 358, L71

[663] Kaspi, V. M., et al. 1999, ApJ, 525, L33

[664] Kato, T., et al. 1995, PASJ, 47, 31

[665] Kato, T., et al. 1999, IBVS, 4777

[666] Kawai, N., et al. 1988, ApJ, 330, 130

[667] Kawai, N., et al. 1990, PASJ, 42, 115

[668] Kellogg, E., et al. 1971, ApJ, 169, L99

[669] Kennea, J. A., \& Skinner, G. K. 1996, PASJ, 48, L117

[670] Kesteven, M. J., \& Turtle, A. J. 1991, IAU Circ., 5181

[671] Kholopov, P. N., et al. 1989, IBVS, 3323

[672] Kii, S., et al. 1986, PASJ, 38, 751

[673] King, A. R. 1993, MNRAS, 260, L5 
[674] King, I. R., et al. 1993, ApJ, 413, L117

[675] King, N. L., et al. 1996, AJ, 111, 1675

[676] Kitamoto, S., et al. 1984, PASJ, 36, 799

[677] Kitamoto, S., et al. 1989, Nature, 342, 518

[678] Kitamoto, S., et al. 1990, ApJ, 361, 590

[679] Kitamoto, S., et al. 1992, ApJ, 391, 220

[680] Kitamoto, S., et al. 1992, ApJ, 394, 609

[681] Kitamoto, S., et al. 1993, ApJ, 403, 315

[682] Kleinmann, D. E., et al. 1978, ApJ, 210, L83

[683] Ko, Y. K., et al. 1999, ApJ, 520, 292

[684] Koch, M. L., \& Naylor, T. 1995, A\&A, 296, 390

[685] Kommers, J. M., et al. 1997, ApJ, 482, L53

[686] Kondo, Y., et al. 1983, ApJ, 273, 716

[687] Kong, A. K. H., et al. 2000, MNRAS, 312, L49

[688] Koo, D. C., \& Kron, R. G. 1977, PASP, 89, 285

[689] Koshut, T. M., et al. 1998, ApJ, 496, L101

[690] Kotani, T., et al. 1999, ApJ, 510, 369

[691] Kotani, T., et al. 2000, ApJ, 539, 413

[692] Kouveliotou, C., et al. 1992, IAU Circ., 5579

[693] Kouveliotou, C., et al. 1996, Nature, 379, 799

[694] Kouveliotou, C., et al. 1996, IAU Circ., 6286

[695] Kouveliotou, C., et al. 1997, IAU Circ., 6530

[696] Kouveliotou, C., et al. 1998, Nature, 393, 235

[697] Kouveliotou, C., et al. 1999, ApJ, 510, L115

[698] Koyama, K., et al. 1981, ApJ, 247, L27

[699] Koyama, K., et al. 1987, PASJ, 39, 801

[700] Koyama, K., et al. 1989, PASJ, 41, 461

[701] Kraft, R. P., \& Miller, J. S. 1969, ApJ, 155, L159

[702] Kroeger, R. A., et al. 1996, A\&AS, 120, 117

[703] Krzeminski, W., \& Kubiak, M. 1991, Acta Astr., 41, 117

[704] Kubota, A., et al. 1998, PASJ, 50, 667

[705] Kuchinski, L. E., \& Frogel, J. A. 1995, AJ, 110, 2844

[706] Kulkarni, S. R., \& van Kerkwijk, M. H. 1997, ApJ, 507, L49

[707] Kunieda, H., et al. 1984, PASJ, 36, 215

[708] Kunieda, H., et al. 1984, PASJ, 36, 807

[709] Kuulkers, E., \& van der Klis, M. 1996, A\&A, 314, 567

[710] Kuulkers, E., \& van der Klis, M. 1998, A\&A, 332, 845

[711] Kuulkers, E., \& van der Klis, M. 2000, A\&A, 356, L45

[712] Kuulkers, E., et al. 1994, A\&A, 289, 795

[713] Kuulkers, E., et al. 1997, MNRAS, 287, 495

[714] Kuulkers, E., et al. 1995, ApJ, 450, 748

[715] Kuulkers, E., et al. 1997, A\&A, 323, L29

[716] Kuulkers, E., et al. 1997, ApJ, 474, L47

[717] Kuulkers, E., et al. 1998, ApJ, 494, 753

[718] Kuulkers, E., et al. 1999, MNRAS, 306, 919

[719] Kuulkers, E., et al. 1999, MNRAS, 308, 485

[720] Kuulkers, E., et al. 2000, A\&A, 358, 993

[721] Langmeier, A., et al. 1985, Sp. Sci. Rev., 40, 367

[722] Langmeier, A., et al. 1987, ApJ, 323, 288

[723] Langmeier, A., et al. 1989, ApJ, 340, L21

[724] Langmeier, A., et al. 1990, A\&A, 228, 89

[725] Lapshov, I., et al. 1992, SvA Lett., 18, 12

[726] Laros, J., \& Wheaton, W. A. 1980, Nature, 284, 324

[727] LaSala, J., \& Thorstensen, J. R. 1985, AJ, 90, 2077

[728] Laurent, P., et al. 1993, A\&A, 278, 444

[729] Lawrence, A., et al. 1983, ApJ, 267, 301

[730] Lawrence, A., et al. 1983, ApJ, 271, 793

[731] Leahy, D. A. 1991, MNRAS, 251, 22P

[732] Leahy, D. A. 2000, MNRAS, 315, 735

[733] Leahy, D. A., \& Marshall, H. 1999, ApJ, 521, 328

[734] Leahy, D. A., et al. 1992, A\&A, 259, 209

[735] Lehto, H., et al. 1990, Nature, 347, 49

[736] Leibowitz, E. M., et al. 1991, MNRAS, 250, 385
[737] Leibowitz, E. M., et al. 1998, MNRAS, 300, 463

[738] Levine, A., et al. 1988, ApJ, 327, 732

[739] Levine, A. M., et al. 1996, ApJ, 469, L33

[740] Levine, A. M., et al. 1998, IAU Circ., 6955

[741] Levinson, A., \& Mattox, J. R. 1996, ApJ, 462, L67

[742] Lewin, W. H. G. 1977, Am. Scient., 65, 605

[743] Lewin, W. H. G., \& van Paradijs, J. 1985, A\&A, 142, 361

[744] Lewin, W. H. G., et al. 1971, ApJ, 169, L17

[745] Lewin, W. H. G., et al. 1976, ApJ, 207, L95

[746] Lewin, W. H. G., et al. 1976, IAU Circ., 2994

[747] Lewin, W. H. G., et al. 1976, MNRAS, 177, 83p

[748] Lewin, W. H. G., et al. 1976, MNRAS, 177, 93p

[749] Lewin, W. H. G., et al. 1978, IAU Circ., 3193

[750] Lewin, W. H. G., et al. 1980, MNRAS, 193, 15

[751] Lewin, W. H. G., et al. 1987, MNRAS, 226, 383

[752] Lewin, W. H. G., et al. 1987, ApJ, 319, 893

[753] Lewin, W. H. G., et al. 1988, Sp. Sci. Rev., 46, 273

[754] Lewin, W. H. G., et al. 1991, A\&A, 248, 538

[755] Lewin, W. H. G., et al. 1992, MNRAS, 256, 545

[756] Lewin, W. H. G., et al. 1995, X-ray Binaries (Cambridge University Press)

[757] Lewin, W. H. G., et al. 1996, ApJ, 462, L39

[758] Li, F. K., \& Clark, G. W. 1977, IAU Circ., 3095

[759] Li, F. K., et al. 1976, ApJ, 203, 187

[760] Li, F. K., et al. 1978, Nature, 276, 799

[761] Li, F. K., et al. 1979, MNRAS, 179, 21p

[762] Li, F. K., et al. 1980, ApJ, 240, 628

[763] Li, X. D., \& van den Heuvel, E. P. J. 1997, A\&A, 321, L25

[764] Li, X. D., et al. 1995, A\&A, 303, L1

[765] Li, X. D., et al. 1999, PhRvL, 83, 3776

[766] Li, X. D., et al. 1999, ApJ, 527, L51

[767] Liller, W. 1977, ApJ, 213, L21

[768] Liller, W. 1999, IAU Circ., 7254

[769] Lin, D., et al. 2000, ApJ, 532, 548

[770] Liu, Q. Z., et al. 2000, A\&AS, 147, 25

[771] Lloyd, C., et al. 1979, MNRAS, 179, 675

[772] Lochner, J. C., \& Roussel-Dupre, D. 1990, BAAS, 22, 804

[773] Lochner, J. C., \& Roussel-Dupre, D. 1994, ApJ, 435, 840

[774] Long, K. S., et al. 1981, ApJ, 248, 925

[775] Lubin, L. M., et al. 1991, MNRAS, 249, 300

[776] Lubin, L. M., et al. 1991, MNRAS, 252, 190

[777] Lubin, L. M., et al. 1992, MNRAS, 256, 624

[778] Lucke, P. 1974, ApJS, 28, 73

[779] Lugger, P. M., et al. 1987, ApJ, 320, 482

[780] Lum, K. S. K., et al. 1992, ApJS, 78, 423

[781] Lund, N., et al. 1991, IAU Circ., 5161

[782] Lund, N., et al. 1991, Adv. Space Res., 11(8), 17

[783] Lyutyi, V. M., \& Shugarov, S. Yu. 1979, SvA Lett., 5, 206

[784] Lyutyi, V. M., \& Voloshina, I. B. 1989, SvA Lett., 15, 347

[785] Machin, G., et al. 1990, MNRAS, 246, 237

[786] Machin, G., et al. 1990, MNRAS, 247, 205

[787] Madsen, J. 1997, A\&A, 318, 466

[788] Maeda, Y., et al. 1996, PASJ, 48, 417

[789] Maejima, Y., et al. 1984, ApJ, 285, 712

[790] Magnier, E., et al. 1989, MNRAS, 237, 729

[791] Main, D. S., et al. 1999, ApJ, 525, 901

[792] Makino, F. 1988, IAU Circ., 4571

[793] Makino, F., \& the Ginga Team, 1988, IAU Circ., 4653

[794] Makino, F., et al. 1990, IAU Circ., 5142

[795] Makino, F., et al. 1990, IAU Circ., 5148

[796] Makishima, K., et al. 1981, ApJ, 244, L79 
[797] Makishima, K., et al. 1981, ApJ, 247, L23

[798] Makishima, K., et al. 1982, ApJ, 255, L49

[799] Makishima, K., et al. 1983, ApJ, 267, 310

[800] Makishima, K., et al. 1986, ApJ, 308, 635

[801] Makishima, K., et al. 1988, Nature, 333, 746

[802] Makishima, K., et al. 1989, PASJ, 41, 531

[803] Malkan, M., et al. 1980, ApJ, 237, 432

[804] Manchanda, R. K., et al. 1995, A\&A, 293, L29

[805] Mandrou, P., et al. 1990, IAU Circ., 5140

[806] Maraschi, L., et al. 1980, ApJ, 241, L23

[807] Margon, B. 1978, ApJ, 219, 613

[808] Margon, B., et al. 1978, Nature, 271, 63

[809] Margon, B., et al. 1978, ApJ, 221, 907

[810] Markert, T. H., et al. 1973, ApJ, 184, L67

[811] Markert, T. H., et al. 1975, Nature, 257, 32

[812] Markert, T. H., et al. 1976, ApJ, 208, L115

[813] Markert, T. H., et al. 1977, ApJ, 218, 801

[814] Markert, T. H., et al. 1979, ApJS, 39, 573

[815] Markwardt, C. B., \& Swank, J. H. 2000, IAU Circ., 7454

[816] Markwardt, C. B., et al. 1998, IAU Circ., 6998

[817] Markwardt, C. B., et al. 1999, ApJ, 512, L125

[818] Markwardt, C. B., et al. 1999, IAU Circ., 7274

[819] Markwardt, C. B., et al. 2000, Astrophys. with RXTE, 7

[820] Marsh, T. R., et al. 1994, MNRAS, 266, 137

[821] Marshall, F. E., \& Markwardt, C. B. 1999, IAU Circ., 7103

[822] Marshall, F. E., \& Smith, D. M. 1997, IAU Circ., 6603

[823] Marshall, F. E., \& Strohmayer, T. 1998, IAU Circ., 6922

[824] Marshall, F. E., et al. 1996, IAU Circ., 6504

[825] Marshall, F. E., et al. 1997, IAU Circ., 6543

[826] Marshall, F. E., et al. 1998, IAU Circ., 6891

[827] Marshall, F. E., et al. 1998, IAU Circ., 6876

[828] Marshall, F. E., et al. 1999, IAU Circ., 7133

[829] Marshell, H. L., et al. 1979, ApJ, 227, 555

[830] Marshall, N., \& Millit, J. M. 1981, Nature, 293, 379

[831] Marti, J., et al. 1997, A\&A, 323, 158

[832] Marti, J., et al. 1998, A\&A, 332, L45

[833] Marti, J., et al. 1998, A\&A, 338, L95

[834] Martin, A. C., et al. 1995, MNRAS, 274, L46

[835] Martin, A. C., et al. 1995, MNRAS, 274, 559

[836] Martin, E. L., et al. 1992, Nature, 358, 129

[837] Martins, D. H., et al. 1980, AJ, 85, 521

[838] Masetti, N., et al. 1996, A\&A, 314, 123

[839] Masetti, N., et al. 1997, A\&A, 317, 769

[840] Mason, K. O., \& Cordova, F. A. 1982, ApJ, 255, 603

[841] Mason, K. O., \& Cordova, F. A. 1982, ApJ, 262, 253

[842] Mason, K. O., et al. 1980, ApJ, 242, L109

[843] Mason, K. O., et al. 1980, Nature, 287, 516

[844] Mason, K. O., et al. 1982, MNRAS, 200, 793

[845] Mason, K. O., et al. 1985, MNRAS, 216, 1033

[846] Mason, K. O., et al. 1985, Sp. Sci. Rev., 40, 225

[847] Mason, K. O., et al. 1987, MNRAS, 226, 423

[848] Matilsky, T. A., et al. 1972, ApJ, 174, L53

[849] Matilsky, T., et al. 1976, ApJ, 210, L127

[850] Matsuba, E., et al. 1995, PASJ, 47, 575

[851] Matsuoka, M. 1980, Symp. Space Ap., (ISAS), 88

[852] Matsuoka, M., et al. 1980, ApJ, 240, L137

[853] Matsuoka, M., et al. 1984, ApJ, 283, 774

[854] Mauche, C., et al. 2000, IAU Circ., 7401

[855] Mavromatakis, F. 1994, A\&A, 285, 503

[856] McClintock, J. E., \& Leventhal, M. 1989, ApJ, 346, 143

[857] McClintock, J. E., \& Remillard, R. A. 1986, ApJ, 308, 110
[858] McClintock, J. E., \& Remillard, R. A. 1990, ApJ, 350, 386

[859] McClintock, J. E., \& Remillard, R. A. 2000, ApJ, 531, 956

[860] McClintock, J., et al. 1978, IAU Circ., 3251

[861] McClintock, J. E., et al. 1978, Nature, 270, 320

[862] McClintock, J. E., et al. 1978, ApJ, 223, L75

[863] McClintock, J. E., et al. 1979, Nature, 279, 47

[864] McClintock, J. E., et al. 1981, ApJ, 243, 900

[865] McClintock, J. E., et al. 1982, ApJ, 258, 245

[866] McClintock, J. E., et al. 1984, ApJ, 283, 794

[867] McClintock, J. E., et al. 1992, IAU Circ., 5499

[868] McClintock, J. E., et al. 1998, IAU Circ., 7025

[869] McClintock, J. E., et al. 1995, ApJ, 442, 358

[870] McCollough, M. L., \& Wilson, C. A. 1999, IAU Circ., 7282

[871] McCroskey, R. E. 1992, IAU Circ., 5597

[872] McHardy, I. M., et al. 1981, MNRAS, 197, 893

[873] McNamara, B. J., et al. 1998, ApJS, 116, 287

[874] Meier, D. 1996, ApJ, 459, 185

[875] Mendez, M., \& van der Klis, M. 1999, ApJ, 517, L51

[876] Mendez, M., et al. 1997, ApJ, 485, L37

[877] Mendez, M., et al. 1998, ApJ, 494, L65

[878] Mendez, M., et al. 1998, ApJ, 499, L187

[879] Mendez, M., et al. 1998, ApJ, 503, L23

[880] Mendez, M., et al. 1998, ApJ, 506, L117

[881] Mereghetti, S., \& Stella, L. 1995, ApJ, 442, L17

[882] Mereghetti, S., et al. 1992, A\&A, 259, 205

[883] Mereghetti, S., et al. 1994, ApJ, 433, L21

[884] Mereghetti, S., et al. 1995, A\&A, 302, 713

[885] Mereghetti, S., et al. 1992, A\&A, 263, 172

[886] Mereghetti, S., et al. 1997, A\&A, 321, 835

[887] Mereghetti, S., et al. 1997, ApJ, 476, 829

[888] Mereghetti, S., et al. 1998, MNRAS, 296, 689

[889] Middleditch, J. 1983, ApJ, 275, 278

[890] Middleditch, J., \& Priedhorsky, W. C. 1986, ApJ, 306, 230

[891] Middleditch, J., et al. 1981, ApJ, 244, 1001

[892] Middleditch, J., et al. 1983, ApJ, 274, 313

[893] Middleditch, J., et al. 1984, ApJ, 292, 267

[894] Mihara, T., et al. 1990, Nature, 346, 250

[895] Mihara, T., et al. 1991, PASJ, 43, 501

[896] Mineshige, S., et al. 1992, PASJ, 44, 117

[897] Mirabel, I. F., \& Rodriguez, L. F. 1994, Nature, 371, 46

[898] Mirabel, I. F., \& Rodriguez, L. F. 1995, Ap\&SS, 231, 65

[899] Mirabel, I. F., et al. 1991, A\&A, 251, L43

[900] Mirabel, I. F., et al. 1992, Nature, 358, 215; IAU Circ., 5655

[901] Mirabel, I. F., et al. 1993, IAU Circ., 5876

[902] Mirabel, I. F., et al. 1996, IAU Circ., 6427

[903] Mirabel, I. F., et al. 1996, ApJ, 472, L111

[904] Mirabel, I. F., et al. 1997, ApJ, 477, L45

[905] Mirabel, I. F., et al. 1998, A\&A, 330, L9

[906] Mironov, A. V. 1986, SvA, 30, 68

[907] Mitsuda, K., \& Dotani, T. 1989, PASJ, 41, 557

[908] Mitsuda, K., et al. 1989, PASJ, 41, 97

[909] Mitsuda, K., et al. 1990, ApJ, 353, 480

[910] Mitsuda, K., et al. 1991, PASJ, 43, 113

[911] Miyamoto, S., \& Matsuda, M. 1977, Sp. Sci. Rev., 20, 687

[912] Miyamoto, S., et al. 1991, ApJ, 383, 784

[913] Miyamoto, S., et al. 1992, ApJ, 391, L21

[914] Molkov, S. V., et al. 2000, A\&A, 357, L41

[915] Molnar, L. A., \& Neely, M. 1992, IAU Circ., 5595

[916] Moneti, A. 1992, A\&A, 260, L7 
[917] Mony, B., et al. 1991, A\&A, 247, 405

[918] Mook, D. E., et al. 1975, ApJ, 197, 425

[919] Moore, C. B., et al. 2000, ApJ, 532, 1181

[920] Morgan, E. H., et al. 1997, ApJ, 482, 993

[921] Morris, S. L., et al. 1990, ApJ, 365, 686

[922] Motch, C., \& Haberl, F. 1998, A\&A, 333, L59

[923] Motch, C., \& Pakull, M. W. 1989, A\&A, 214, L1

[924] Motch, C., et al. 1982, A\&A, 109, L1

[925] Motch, C., et al. 1983, A\&A, 119, 171

[926] Motch, C., et al. 1985, Sp. Sci. Rev., 40, 219

[927] Motch, C., et al. 1987, ApJ, 313, 792

[928] Motch, C., et al. 1989, A\&A, 219, 158

[929] Motch, C., et al. 1989, in Ref. [1421], 545

[930] Motch, C., et al. 1994, IAU Circ., 6101

[931] Motch, C., et al. 1998, A\&AS, 132, 341

[932] Mukai, K., \& Smale, A. P. 2000, ApJ, 533, 352

[933] Muller, J. M., et al. 1998, IAU Circ., 6842

[934] Muller, J. M., et al. 1998, IAU Circ., 6867

[935] Murakami, T., et al. 1980, ApJ, 240, L143

[936] Murakami, T., et al. 1980, PASJ, 32, 543

[937] Murakami, T., et al. 1983, PASJ, 35, 531

[938] Murakami, T., et al. 1987, PASJ, 39, 879

[939] Murdin, P., et al. 1974, MNRAS, 169, 25

[940] Murdin, P., et al. 1977, MNRAS, 178, 27p

[941] Murdin, P., et al. 1980, MNRAS, 192, 709

[942] Murdin, P., et al. 1980, A\&A, 87, 292

[943] Nagase, F. 1989, PASJ, 41, 1

[944] Nagase, F., et al. 1984, PASJ, 36, 215

[945] Naik, S., et al. 2000, A\&A, 354, 938

[946] Nakamura, N., et al. 1988, PASJ, 40, 209

[947] Nakamura, N., et al. 1989, PASJ, 41, 617

[948] Natalucci, L., et al. 1999, ApJ, 523, L45

[949] Natalucci, L., et al. 2000, ApJ, 536, 891

[950] Naylor, T., \& Charles, P. A. 1989, MNRAS, 236, 1p

[951] Naylor, T., et al. 1988, MNRAS, 233, 285

[952] Naylor, T., et al. 1991, MNRAS, 252, 203

[953] Naylor, T., et al. 1992, MNRAS, 255, 1

[954] Neckel, T., \& Klare, G. 1980, A\&AS, 42, 251

[955] Nicolson, G. D., et al. 1980, MNRAS, 191, 293

[956] Nieto, J. L., et al. 1990, A\&A, 239, 155

[957] Nishiuchi, M., et al. 1999, ApJ, 517, 436

[958] Norris, J. P., \& Matilsky, T. A. 1989, ApJ, 346, 912

[959] Norris, J. P., \& Wood, K. S. 1987, ApJ, 312, 732

[960] Norris, J. P., et al. 1990, ApJ, 361, 514

[961] Norton, A. J., et al. 1999, IAU Circ., 7279

[962] Nowak, M. A., et al. 1997, ApJ, 477, L91

[963] Nowak, M. A., et al. 1999, ApJ, 517, 355

[964] Ogelman, H. 1987, A\&A, 172, 79

[965] Ogley, R. N., et al. 1997, IAU Circ., 6726

[966] Ohashi, T., et al. 1982, ApJ, 258, 254

[967] Oke, J. B. 1977, ApJ, 217, 181

[968] Okumura, S., \& Noguchi, T. 1989, IAU Circ., 4589

[969] Olive, J. F., et al. 1998, A\&A, 333, 942

[970] Oosterbroek, T., et al. 1991, A\&A, 250, 389

[971] Oosterbroek, T., et al. 1994, A\&A, 281, 803

[972] Oosterbroek, T., et al. 1995, A\&A, 297, 141

[973] Oosterbroek, T., et al. 1996, A\&A, 309, 781

[974] Oosterbroek, T., et al. 1997, A\&A, 321, 776

[975] Oosterbroek, T., et al. 1997, A\&A, 327, 215

[976] Oosterbroek, T., et al. 1998, A\&A, 340, 431

[977] Oosterbroek, T., et al. 1998, A\&A, 334, 925

[978] Oosterbroek, T., et al. 2000, A\&A, 353, 575

[979] Orlandini, M., et al. 1998, ApJ, 500, L163

[980] Orosz, J. A., \& Bailyn, C. D. 1995, ApJ, 446, L59
[981] Orosz, J. A., \& Bailyn, C. D. 1997, ApJ, 477, 876

[982] Orosz, J. A., \& Kuulkers, E. 1999, MNRAS, 305, 132

[983] Orosz, J. A., et al. 1997, ApJ, 478, L83

[984] Orosz, J. A., et al. 1998, ApJ, 499, 375

[985] Orosz, J. A., et al. 2000, IAU Circ., 7440

[986] Ortolani, S., et al. 1994, A\&AS, 108, 653

[987] Ortolani, S., et al. 1994, A\&A, 286, 444

[988] Ortolani, S., et al. 1999, A\&A, 350, 840

[989] Owen, F. N., et al. 1976, ApJ, 203, L15

[990] Owens, A., et al. 1997, A\&A, 324, L9

[991] Paciesas, W. S., et al. 1992, IAU Circ., 5580

[992] Pakull, M. 1978, IAU Circ., 3313

[993] Pakull, M., \& Swings, J. B. 1979, IAU Circ., 3318

[994] Pan, H. C., et al. 1995, MNRAS, 274, L15

[995] Parmar, A. N., et al. 1984, Nature, 313, 119

[996] Parmar, A. N., et al. 1985, IAU Circ., 4058

[997] Parmar, A. N., et al. 1986, ApJ, 304, 664

[998] Parmar, A. N., et al. 1986, ApJ, 308, 199

[999] Parmar, A. N., et al. 1989, A\&A, 222, 96

[1000] Parmar, A. N., et al. 1989, ApJ, 338, 1024

[1001] Parmar, A. N., et al. 1991, ApJ, 366, 253

[1002] Parmar, A. N., et al. 1993, A\&A, 279, 179

[1003] Parmar, A. N., et al. 1995, ApJ, 452, L129

[1004] Parmar, A. N., et al. 1999, A\&A, 350, L5

[1005] Parmar, A. N., et al. 1999, A\&A, 351, 225

[1006] Parmar, A. N., et al. 2000, A\&A, 356, 175

[1007] Patterson, J. 2000, IAU Circ., 7412

[1008] Patterson, T. G., et al. 1989, in Ref. [1421], 567

[1009] Paul, B., et al. 1997, A\&A, 320, L9

[1010] Paul, B., et al. 1997, A\&A, 320, L37

[1011] Paul, B., et al. 1998, A\&AS, 128, 145

[1012] Paul, B., et al. 1998, ApJ, 492, L63

[1013] Paul, B., et al. 2000, ApJ, 528, 410

[1014] Paul, B., et al. 2000, ApJ, 537, 319

[1015] Paul, J. 1991, in $\gamma$-ray Line Astrophys., ed. P. Duchouroux, \& N. Prantzos, AIP Proc., 232, 17

[1016] Paul, J., et al. 1996, IAU Circ., 6348

[1017] Pavlenko, E. P., et al. 1989, SvA Lett., 15, 262

[1018] Pavlenko, E. P., et al. 1996, MNRAS, 281, 1094

[1019] Pavlinsky, M. N., et al. 1992, SvA Lett., 18, 88

[1020] Pavlinsky, M. N., et al. 1994, ApJ, 425, 110

[1021] Pederson, H. 1983, ESO Messenger, 34, 21

[1022] Pederson, H., et al. 1981, Nature, 294, 725

[1023] Pederson, H., et al. 1982, ApJ, 263, 325

[1024] Pederson, H., et al. 1982, ApJ, 263, 340

[1025] Pederson, H., et al. 1983, IAU Circ., 3858

[1026] Penninx, W., \& Augusteijn, Th. 1991, A\&A, 246, L81

[1027] Penninx, W., et al. 1988, Nature, 336, 146

[1028] Penninx, W., et al. 1989, A\&A, 208, 146

[1029] Penninx, W., et al. 1989, MNRAS, 238, 851

[1030] Penninx, W., et al. 1990, A\&A, 240, 317

[1031] Penninx, W., et al. 1990, MNRAS, 243, 114

[1032] Penninx, W., et al. 1991, MNRAS, 249, 113

[1033] Penninx, W., et al. 1993, A\&A, 267, 92

[1034] Percival, W., et al. 1998, IAU Circ., 6902

[1035] Pereira, M. G., et al. 1999, ApJ, 526, L105

[1036] Peterson, R. C., et al. 1989, ApJ, 347, 251

[1037] Petro, L. D., et al. 1981, ApJ, 251, L7

[1038] Petterson, J. A., et al. 1975, ApJ, 201, L61

[1039] Petterson, J. A., et al. 1977, ApJ, 216, 827

[1040] Phillips, S. N., et al. 1999, MNRAS, 304, 839

[1041] Pietsch, W., et al. 1986, A\&A, 157, 23

[1042] Pietsch, W., et al. 1993, A\&A, 273, L11

[1043] Piraino, S., et al. 1999, A\&A, 349, L77 
[1044] Podsiadlowski, Ph., \& Rappaport, S. 2000, ApJ, 529, 946

[1045] Ponman, T. J., et al. 1988, MNRAS, 231, 999

[1046] Pooley, G. G. 1998, IAU Circ., 6924

[1047] Pooley, G. G., \& Fender, R. P. 1997, MNRAS, 292, 925

[1048] Pooley, G. G., \& Hjellming, R. M. 1999, IAU Circ., 7278

[1049] Pooley, G. G., \& Waldram, E. M. 2000, IAU Circ., 7390

[1050] Pravdo, S. H., et al. 1979, ApJ, 231, 912

[1051] Predehl, P., \& Truemper, J. 1994, A\&A, 290, L29

[1052] Predehl, P., et al. 1991, A\&A, 246, L21

[1053] Predehl, P., et al. 1991, A\&A, 246, L40

[1054] Predehl, P., et al. 1995, A\&A, 293, L29

[1055] Preston, R. A., et al. 1983, ApJ., 268, L23

[1056] Priedhorsky, W. 1986, Ap\&SS, 126, 89

[1057] Priedhorsky, W. C., \& Holt, S. S. 1987, Sp. Sci. Rev., 45, 291

[1058] Priedhorsky, W. C., \& Holt, S. S. 1987, ApJ, 312, 743

[1059] Priedhorsky, W., \& Terrell, J. 1984, ApJ, 280, 661

[1060] Priedhorsky, W., \& Terrell, J. 1984, ApJ, 284, L17

[1061] Priedhorsky, W., et al. 1986, ApJ, 306, L91

[1062] Prince, T., et al. 1991, IAU Circ., 5252

[1063] Prins, S., \& van der Klis, M. 1997, A\&A, 319, 498

[1064] Proctor, R. J., et al. 1978, MNRAS, 185, 745

[1065] Pryor, C., et al. 1990, AJ, 98, 596

[1066] Puchnarewicz, E. M., et al. 1995, Adv. Sp. Rev., 16, 65

[1067] Qiao, G. \& Cheng, J. 1989, ApJ, 340, 503

[1068] Rao, A. R., et al. 1994, A\&A, 289, L43

[1069] Rappaport, S., \& Joss, P. C. 1997, ApJ, 486, 435

[1070] Rappaport, S. A., et al. 1977, Nature, 268, 705

[1071] Reich, W., \& Schlickeiser, R. 1992, A\&A, 256, 408

[1072] Reid, C. A., et al. 1980, AJ, 85, 1062

[1073] Reig, P., et al. 2000, ApJ, 530, 916

[1074] Remillard, R. 1998, IAU Circ., 6983

[1075] Remillard, R. E., et al. 1992, ApJ, 399, L145

[1076] Remillard, R. A., et al. 1996, IAU Circ., 6393

[1077] Remillard, R., et al. 1996, ApJ, 459, 226

[1078] Remillard, R., et al. 1997, IAU Circ., 6710

[1079] Remillard, R., et al. 1998, IAU Circ., 6920

[1080] Remillard, R. A., et al. 1998, IAU Circ., 7019

[1081] Remillard, R. A., et al. 1999, IAU Circ., 7123

[1082] Remillard, R. A., et al. 1999, ApJ, 517, L127

[1083] Remillard, R. A., et al. 1999, ApJ, 522, 397

[1084] Remillard, R., et al. 2000, IAU Circ., 7389

[1085] Revnivtsev, M., et al. 1998, A\&A, 331, 557

[1086] Revnivtsev, M., et al. 1998, A\&A, 339, 483

[1087] Revnivtsev, M., et al. 1999, A\&A, 344, L25

[1088] Revnivtsev, M. G., et al. 2000, MNRAS, 312, 151

[1089] Revnivtsev, M. G., et al. 2000, ApJ, 530, 955

[1090] Revnivtsev, M., et al. 2000, A\&A, 361, L37

[1091] Reynolds, A. P., et al. 1997, MNRAS, 288, 43

[1092] Ricci, D., et al. 1995, A\&A, 299, 731

[1093] Richer, G. A., et al. 1987, IBVS, 3362

[1094] Ricker, G. R., et al. 1973, ApJ, 184, 237

[1095] Robertson, B. S. C., et al. 1976, IBVS, 1173

[1096] Robinson, E. L., \& Yough, P. 1997, ApJ, 491, L89

[1097] Roche, P., et al. 1998, IAU Circ., 6885

[1098] Rodriguez, L. F., \& Mirabel, I. F. 1997, ApJ, 474, L123

[1099] Rodriguez, L. F., \& Mirabel, I. F. 1998, A\&A, 340, L47

[1100] Rodriguez, L. F., \& Mirabel, I. F. 1999, ApJ, 511, 398

[1101] Rodriguez, L. F., et al. 1992, ApJ, 401, L15

[1102] Rodriguez, L. F., et al. 1995, ApJS, 101, 173

[1103] Rothschild, R. E., et al. 1980, Nature, 286, 786

[1104] Rubin, B. C., et al. 1998, ApJ, 492, L67

[1105] Rupen, M. P., et al. 1998, IAU Circ., 6938
[1106] Rutledge, R. E., et al. 1995, MNRAS, 277, 523

[1107] Rutledge, R. E., et al. 1999, ApJS, 124, 265

[1108] Rutledge, R. E., et al. 1999, ApJ, 514, 945

[1109] Sagar, R., et al. 1988, MNRAS, 232, 131

[1110] Sakano, M., et al. 1999, ApJ, 520, 316

[1111] Sakao, T., et al. 1990, MNRAS, 246, 11p

[1112] Sams, B. J., et al. 1996, Nature, 382, 47

[1113] Samus, N. N., et al. 1999, IAU Circ., 7277

[1114] Sanchez-Fernandez, C., et al. 1999, A\&A, 348, L9

[1115] Sandage, A. R., et al. 1966, ApJ, 146, 316

[1116] Sansom, A. E., et al. 1989, PASJ, 41, 591

[1117] Sansom, A. E., et al. 1993, MNRAS, 262, 429

[1118] Sanwal, D., et al. 1996, ApJ, 460, 437

[1119] Sasaki, M., et al. 2000, A\&AS, 143, 391

[1120] Sazonov, S., \& Sunyaev, R. 1996, IAU Circ., 6291

[1121] Scaltriti, F., et al. 1997, A\&A, 325, L29

[1122] Schachter, J., et al. 1989, ApJ, 340, 1049 [erratum: ApJ, $362,379]$

[1123] Schaefer, B. E. 1990, ApJ, 354, 720

[1124] Schoelkopf, R. J., \& Kelley, R. L. 1991, ApJ, 375, 696

[1125] Schoembs, R., \& Zoeschinger, G. 1990, A\&A, 227, 105

[1126] Schmidtke, P. 1988, AJ, 95, 1528

[1127] Schmidtke, P. 1990, PASP, 102, 144

[1128] Schmidtke, P., \& Cowley, A. P. 1987, AJ, 93, 372

[1129] Schmidtke, P., \& Cowley, A. P. 1992, IAU Circ., 5451

[1130] Schulz, N. S. 1999, ApJ, 511, 304

[1131] Schulz, N. S., et al. 1989, A\&A, 225, 48

[1132] Schwentker, O. 1994, A\&A, 286, L47

[1133] Scott, D. M., \& Leahy, D. A. 1999, ApJ, 510, 974

[1134] Seon, K. I., et al. 1995, ApJ, 454, 463

[1135] Seward, F. D., et al. 1976, MNRAS, 177, 13P

[1136] Seward, F. D., et al. 1986, ApJ, 305,814

[1137] Shahbaz, T., et al. 1993, MNRAS, 265, 655

[1138] Shahbaz, T., et al. 1994, MNRAS, 268, 756

[1139] Shahbaz, T., et al. 1994, MNRAS, 271, L10

[1140] Shahbaz, T., et al. 1996, MNRAS, 282, L47

[1141] Shahbaz, T., et al. 1996, MNRAS, 282, 977

[1142] Shahbaz, T., et al. 1996, MNRAS, 282, 1437

[1143] Shahbaz, T., et al. 1997, A\&A, 326, L5

[1144] Shahbaz, T., et al. 1997, MNRAS, 285, 607

[1145] Shahbaz, T., et al. 1998, MNRAS, 296, 1004

[1146] Shahbaz, T., et al. 1998, MNRAS, 300, 1035

[1147] Shahbaz, T., et al. 1999, MNRAS, 306, 89

[1148] Shahbaz, T., et al. 1999, A\&A, 344, 101

[1149] Shahbaz, T., et al. 1999, A\&A, 346, 82

[1150] Shahbaz, T., et al. 2000, MNRAS, 314, 747

[1151] Shakhovskaya, N. 1992, IAU Circ., 5590

[1152] Shakura, N. I., et al. 1998, MNRAS, 300, 992

[1153] Share, G., et al. 1978, IAU Circ., 3190

[1154] Shawl, S. J., \& White, R. E. 1980, ApJ, 239, L61

[1155] Sheth, S., et al. 1996, ApJ, 468, 755

[1156] Shinoda, K., et al. 1990, PASJ, 42, L27

[1157] Shirey, R. E., et al. 1996, ApJ, 469, L21

[1158] Shrader, C. R., et al. 1992, IAU Circ., 5591

[1159] Shrader, C. R., et al. 1994, ApJ, 434, 698

[1160] Shrader, C. R., et al. 1997, ApJ, 487, 858

[1161] Sidoli, L., et al. 1998, A\&A, 336, L81

[1162] Sidoli, L., et al. 1999, ApJ, 525, 215

[1163] Singh, K. P., \& Apparao, K. M. V. 1994, ApJ, 431, 826

[1164] Singh, K., et al. 1994, ApJ, 421, 753

[1165] Skinner, G. K. 1991, in $\gamma$-ray Line Astrophys., ed. P.

Duchouroux, \& N. Prantzos, AIP Proc., 232, 358

[1166] Skinner, G. K., et al. 1987, Nature, 330, 544

[1167] Skinner, G. K., et al. 1989, IAU Circ., 4879 
[1168] Skinner, G. K., et al. 1990, MNRAS, 243, 72

[1169] Skinner, G. K., et al. 1991, A\&A, 252, 172

[1170] Smale, A. P. 1991, PASP, 103, 636

[1171] Smale, A. P. 1998, ApJ, 498, L141

[1172] Smale, A. P., \& Corbet, R. H. D. 1991, ApJ, 383, 853

[1173] Smale, A. P., \& Kuulkers, E. 2000, ApJ, 528, 702

[1174] Smale, A. P., \& Mukai, K. 1988, MNRAS, 231, 663

[1175] Smale, A. P., \& Wachter, S. 1999, ApJ, 527, 341

[1176] Smale, A. P., et al. 1984, MNRAS, 207, 29p

[1177] Smale, A. P., et al. 1986, MNRAS, 223, 207

[1178] Smale, A. P., et al. 1987, MNRAS, 225, 7p

[1179] Smale, A. P., et al. 1988, MNRAS, 232, 647

[1180] Smale, A. P., et al. 1989, in Ref. [1421], 607

[1181] Smale, A. P., et al. 1992, ApJ, 400, 330

[1182] Smale, A. P., et al. 1992, ApJ, 395, 582

[1183] Smale, A. P., et al. 1993, ApJ, 410, 796

[1184] Smale, A. P., et al. 1997, ApJ, 483, L119

[1185] Smale, A. P., et al. 2000, Astrophys. with the RXTE,

10

[1186] Smith, D. A., et al. 1997, ApJ, 479, L137

[1187] Smith, D. A., et al. 1998, IAU Circ., 6932

[1188] Smith, D. A., et al. 1998, IAU Circ., 7008

[1189] Smith, D. A., et al. 1999, IAU Circ., 7253

[1190] Smith, D. M., et al. 1997, ApJ, 489, L51

[1191] Smith, I. A., \& Liang, E. P. 1999, ApJ, 519, 771

[1192] Smith, I. A., et al. 1999, ApJ, 519, 762

[1193] Smith, I. A., et al. 1999, ApJ, 519, 779

[1194] Sobczak, G. J., et al. 1999, ApJ, 517, L121

[1195] Sobczak, G. J., et al. 1999, ApJ, 520, 776

[1196] Sobczak, G. J., et al. 2000, ApJ, submitted

[1197] Song, L., et al. 2000, PASJ, 52, 181

[1198] Soong, Y., et al. 1987, ApJ, 319, L77

[1199] Soong, Y., et al. 1990, ApJ, 348, 634

[1200] Soong, Y., et al. 1990, ApJ, 348, 641

[1201] Soria, R., et al. 1997, IAU Circ., 6781

[1202] Soria, R., et al. 1998, ApJ, 495, L95

[1203] Soria, R., et al. 1999, MNRAS, 309, 528

[1204] Soria, R., et al. 1999, MNRAS, 310, 71

[1205] Sosin, C., \& Cool, A. M. 1995, ApJ, 452, L29

[1206] Sosin, C., \& King, I. R. 1995, AJ, 109, 639

[1207] Southwell, K. A., et al. 1996, IAU Colloq., 158, 365

[1208] Stark, M. J., et al. 1996, ApJ, 470, L109

[1209] Steiman-Cameron, T., et al. 1990, ApJ, 359, 197

[1210] Steiman-Cameron, T. Y., et al. 1997, ApJ, 487, 396

[1211] Stella, L. 1990, Nature, 344, 747

[1212] Stella, L., et al. 1984, ApJ, 282, 713

[1213] Stella, L., et al. 1987, ApJ, 312, L17

[1214] Stella, L., et al. 1987, ApJ, 315, L49

[1215] Stella, L., et al. 1988, ApJ, 324, 379

[1216] Stewart, R. T., et al. 1991, MNRAS, 253, 212

[1217] Stewart, R. T., et al. 1993, MNRAS, 261, 593

[1218] Strickman, M. S., et al. 1996, ApJ, 464, L131

[1219] Strohmayer, T. E. 1999, ApJ, 523, L51

[1220] Strohmayer, T., \& Marshall, F. E. 1998, IAU Circ., 6934

[1221] Strohmayer, T. E., et al. 1996, ApJ, 469, L9

[1222] Strohmayer, T. E., et al. 1997, ApJ, 486, 355

[1223] Strohmayer, T. E., et al. 1997, ApJ, 487, L77

[1224] Stubbings, R. 1999, IAU Circ., 7253

[1225] Sturner, S. J., \& Dermer, C. D. 1996, ApJ, 465, L31

[1226] Sugimoto, D., et al. 1984, PASJ, 36, 839

[1227] Sugizaki, M., et al. 1997, PASJ, 49, L25

[1228] Sunyaev, R. 1990, IAU Circ., 5104

[1229] Sunyaev, R. A., et al. 1988, SvA Lett., 14, 327

[1230] Sunyaev, R. A., et al. 1989, in Ref. [1421], 641
[1231] Sunyaev, R. A., et al. 1990, SvA Lett., 16, 55

[1232] Sunyaev, R. A., et al. 1990, SvA Lett., 16, 59

[1233] Sunyaev, R. A., et al. 1991, SvA Lett., 17, 42

[1234] Sunyaev, R. A., et al. 1991, SvA Lett., 17, 123

[1235] Sunyaev, R. A., et al. 1991, IAU Circ., 5176

[1236] Sunyaev, R. A., et al. 1991, ApJ, 383, L49

[1237] Sunyaev, R. A., et al. 1991, A\&A, 247, L29

[1238] Sunyaev, R. A., et al. 1991, SvA Lett., 17, 50 \& 54

[1239] Sunyaev, R. A., et al. 1991, IAU Circ., 5180

[1240] Sunyaev, R. A., et al. 1991, Adv. Space Res., 11(8), 177

[1241] Sunyaev, R. A., et al. 1992, IAU Circ., 5398

[1242] Sunyaev, R. A., et al. 1992, ApJ, 389, L75

[1243] Sunyaev, R. A., et al. 1993, A\&A, 280, L1

[1244] Sunyaev, R., et al. 1997, IAU Circ., 6599

[1245] Suzuki, K., et al. 1984, PASJ, 36, 761

[1246] Swank, J. 1996, IAU Circ., 6291

[1247] Swank, J. H., et al. 1976, IAU Circ., 3000

[1248] Swank, J. H., et al. 1976, IAU Circ., 3010

[1249] Swank, J. H., et al. 1977, ApJ, 212, L73

[1250] Swank, J. H., et al. 1978, MNRAS, 182, 349

[1251] Swank, J. H., et al. 1984, ApJ, 277, 274

[1252] Sztajno, M., et al. 1983, ApJ, 267, 713

[1253] Sztajno, M., et al. 1985, ApJ, 299, 487

[1254] Sztajno, M., et al. 1986, MNRAS, 222, 499

[1255] Sztajno, M., et al. 1987, MNRAS, 226, 39

[1256] Taam, R. E., et al. 1997, ApJ, 485, L83

[1257] Takeshima, T., \& Strohmayer, T. E. 1998, IAU Circ., 6958

[1258] Takeshima, T., et al. 1996, IAU Circ., 6390

[1259] Takizwa, M., et al. 1997, ApJ, 489, 272

[1260] Tan, J., et al. 1991, MNRAS, 251, 1

[1261] Tan, J., et al. 1991, ApJ, 374, 291

[1262] Tan, J., et al. 1992, ApJ, 385, 314

[1263] Tanaka, Y. 1993, IAU Circ., 5888

[1264] Tananbsum, H., et al. 1976, ApJ, 209, L125

[1265] Tarenghi, M., \& Reina, C. 1972, Nat Phys. Sci., 240, 53

[1266] Tauris, T. M., et al. 1999, MNRAS, 310, 1165

[1267] Tauris, T. M., et al. 2000, ApJ, 530, L93

[1268] Tavani, M., et al. 1996, ApJ, 473, L103

[1269] Tawara, Y., et al. 1982, Nature, 299, 38

[1270] Tawara, Y., et al. 1984, ApJ, 276, L41

[1271] Tawara, Y., et al. 1984, PASJ, 36, 861

[1272] Tawara, Y., et al. 1985, Nature, 318, 545

[1273] Taylor, A. R., et al. 1991, Nature, 351, 547

[1274] Tennant, A. F., 1987, MNRAS, 226, 971

[1275] Tennant, A. F., 1988, MNRAS, 230, 403

[1276] Tennant, A. F., et al. 1986, MNRAS, 219, 871

[1277] Tennant, A. F., et al. 1986, MNRAS, 221, 27p

[1278] Thomas, B., et al. 1993, ApJ, 408, 651

[1279] Thomas, B., et al. 1997, ApJ, 480, L21

[1280] Thompson, C., \& Duncan, R. C. 1996, ApJ, 473, 322

[1281] Thorstensen, J. R. 1987, ApJ, 312, 739

[1282] Thorstensen, J. R., \& Charles, P. A. 1982, ApJ, 253, 756

[1283] Thorstensen, J. R., et al. 1978, ApJ, 220, L131

[1284] Thorstensen, J. R., et al. 1979, ApJ, 233, L57 [erratum 237, L25]

[1285] Thorstensen, J. R., et al. 1980, ApJ, 238, 964

[1286] Thorstensen, J. R., et al. 1988, ApJ, 334, 430

[1287] Tingay, S. J., et al. 1995, Nature, 374, 141

[1288] Tomsella, L., et al. 1997, IBVS, 4537

[1289] Tomsick, J. A., \& Kaaret, P. 2000, ApJ, 537, 448

[1290] Tomsick, J. A., et al. 1998, IAU Circ., 6957

[1291] Tomsick, J. A., et al. 1998, ApJ, 494, 747

[1292] Tomsick, J. A., et al. 1999, ApJ, 512, 892 
[1293] Tomsick, J. A., et al. 1999, ApJ, 521, 341

[1294] Torii, K., et al. 1998, ApJ, 503, 843

[1295] Trudolyubov, S., et al. 1998, A\&A, 334, 895

[1296] Trudolyubov, S., et al. 1999, A\&A, 342, 496

[1297] Trumper, J., et al. 1978, ApJ, 219, L105

[1298] Trumper, J., et al. 1985, Sp. Sci. Rev., 40, 255

[1299] Trumper, J., et al. 1986, ApJ, 300, L63

[1300] Tsunemi, H., et al. 1977, ApJ, 211, L15

[1301] Tsunemi, H., et al. 1989, ApJ, 337, L81

[1302] Tueller, J., et al. 1984, ApJ, 279, 177

[1303] Turner, M. J. L., \& Breedon, L. M. 1984, MNRAS, 208, $29 \mathrm{p}$

[1304] Turner, M. J. L., et al. 1985, Sp. Sci. Rev., 40, 249

[1305] Ubertini, P., et al. 1992, ApJ, 386, 710 [erratum ApJ, $396,378]$

[1306] Ubertini, P., et al. 1999, ApJ, 514, L27

[1307] Ubertini, P., et al. 2000, IAU Circ., 7369

[1308] Udalsky, A., \& Kaluzny, J. 1991, PASP, 103, 198

[1309] Ueda, Y., et al. 1997, IAU Circ., 6627

[1310] Ueda, Y., et al. 1998, ApJ, 492, 782

[1311] Uemura, M., et al. 1999, IAU Circ., 7303

[1312] Uemura, M., et al. 2000, PASJ, 52, L15

[1313] Ulmer, M. P., et al. 1978, Nature, 276, 799

[1314] Ulmer, M. P., et al. 1980, ApJ, 235, L159

[1315] Uno, S., et al. 1997, PASJ, 49, 353

[1316] Usov, V. V. 1994, ApJ, 427, 984

[1317] Vacca, W. D., et al. 1986, MNRAS, 220, 339

[1318] Vacca, W. D., et al. 1987, A\&A, 172, 143

[1319] van Amerongen, S., et al. 1987, A\&A, 185, 147

[1320] van den Heuvel, E. P. J., et al. 1992, A\&A, 262, 97

[1321] van der Hooft, F., et al. 1996, A\&AS, 120, 141

[1322] van der Hooft, F., et al. 1996, ApJ, 458, L75

[1323] van der Hooft, F., et al. 1997, MNRAS, 286, L43

[1324] van der Hooft, F., et al. 1998, A\&A, 329, 538

[1325] van der Hooft, F., et al. 1999, ApJ, 513, 477

[1326] van der Hooft, F., et al. 1999, ApJ, 519, 332

[1327] van der Klis, M. 1989, ARA\&A, 27, 517

[1328] van der Klis, M. 1996, ApJ, 469, L1

[1329] van der Klis, M., et al. 1985, Nature, 316, 225

[1330] van der Klis, M., et al. 1985, Sp. Sci. Rev., 40, 287

[1331] van der Klis, M., et al. 1987, ApJ, 313, L19

[1332] van der Klis, M., et al. 1987, ApJ, 316, 411

[1333] van der Klis, M., et al. 1987, ApJ, 319, L13

[1334] van der Klis, M., et al. 1990, ApJ, 360, L19

[1335] van der Klis, M., et al. 1991, MNRAS, 248, 751

[1336] van der Klis, M., et al. 1993, A\&A, 279, L21

[1337] van der Klis, M., et al. 1993, MNRAS, 260, 686

[1338] van der Klis, M., et al. 1997, ApJ, 481, L97

[1339] van der Klis, M., et al. 2000, IAU Circ., 7358

[1340] van der Woerd, H., et al. 1989, ApJ, 344, 320

[1341] van Dijk, R., et al. 1995, A\&A, 296, L33

[1342] van Paradijs, J. 1983, in Accretion Driven Stellar X-ray Sources, ed. W. H. G. Lewin, \& E. P. J. van den Heuvel (Cambridge), 189

[1343] van Paradijs, J. 1995, in X-ray Binaries, ed. W. H. G. Lewin, et al. (Cambridge), 536

[1344] van Paradijs, J., \& Isaacman, R. 1989, A\&A, 222, 129

[1345] van Paradijs, J., \& Lewin, W. H. G. 1987, A\&A, 172, L20

[1346] van Paradijs, J., et al. 1979, MNRAS, 189, 387

[1347] van Paradijs, J., et al. 1980, ApJ, 241, L161

[1348] van Paradijs, J., et al. 1986, A\&AS, 63, 71

[1349] van Paradijs, J., et al. 1986, MNRAS, 221, 617

[1350] van Paradijs, J., et al. 1987, A\&A, 182, 47
[1351] van Paradijs, J., et al. 1988, A\&A, 192, 147

[1352] van Paradijs, J., et al. 1988, A\&AS, 76, 185

[1353] van Paradijs, J., et al. 1988, MNRAS, 231, 379

[1354] van Paradijs, J., et al. 1990, A\&A, 234, 181

[1355] van Paradijs, J., et al. 1990, A\&A, 235, 156

[1356] van Paradijs, J., et al. 1990, PASJ, 42, 633

[1357] van Paradijs, J., et al. 1994, ApJ, 429, L19

[1358] van Paradijs, J., et al. 1995, A\&A, 299, L41

[1359] van Paradijs, J., et al. 1997, A\&A, 317, L9

[1360] Vargas, M., et al. 1996, A\&A, 313, 828

[1361] Vargas, M., et al. 1997, ApJ, 476, L23

[1362] Vasisht, G., \& Gotthelf, E. V. 1997, ApJ, 486, L129

[1363] Vaughan, B. A., et al. 1994, ApJ, 421, 738

[1364] Vaughan, B. A., et al. 1997, ApJ, 483, L115

[1365] Vaughan, B. A., et al. 1999, A\&A, 343, 197

[1366] Verbunt, F., et al. 1984, MNRAS, 210, 899

[1367] Verbunt, F., et al. 1990, A\&A, 234, 195

[1368] Verbunt, F., et al. 1994, A\&A, 285, 903

[1369] Verbunt, F., et al. 1995, A\&A, 300, 732

[1370] Verbunt, F., et al. 2000, A\&A, 359, 960

[1371] Vilhu, O., \& Nevalainen, J. 1998, ApJ, 508, L85

[1372] Voges, W., et al. 1987, ApJ, 320, 794

[1373] Voges, W., et al. 1996, IAU Circ., 6420

[1374] Vrtilek, S. D., \& Cheng, F. H. 1996, ApJ, 465, 915

[1375] Vrtilek, S. D., et al. 1986, ApJ, 307, 698

[1376] Vrtilek, S. D., et al. 1986, ApJ, 308, 644

[1377] Vrtilek, S. D., et al. 1988, ApJ, 329, 276

[1378] Vrtilek, S. D., et al. 1990, A\&A, 235, 162

[1379] Vrtilek, S. D., et al. 1991, ApJ, 376, 278

[1380] Vrtilek, S. D., et al. 1991, ApJS, 76, 1127

[1381] Vrtilek, S. D., et al. 1994, ApJ, 436, L9

[1382] Wachter, S. 1997, ApJ, 485, 839

[1383] Wachter, S. 1997, ApJ, 490, 401

[1384] Wachter, S., \& Margon, B. 1996, ApJ, 112, 2684

[1385] Wachter, S., \& Smale, A. P. 1998, ApJ, 496, L21

[1386] Wachter, S., et al. 2000, ApJ, 534, 367

[1387] Wade, C. M., \& Hjellming, R. M. 1971, ApJ, 170, 523

[1388] Wagner, R. M. 1999, IAU Circ., 7276

[1389] Wagner, R. M., et al. 1989, IAU Circ., 4783

[1390] Wagner, R. M., et al. 1991, ApJ, 378, 293

[1391] Wagner, R. M., et al. 1992, IAU Circ., 5589

[1392] Wagner, R. M., et al. 1992, ApJ, 401, L97

[1393] Wagner, R., et al. 1994, ApJ, 429, L25

[1394] Wagner, R. M., et al. 1999, IAU Circ., 7279

[1395] Waki, I., et al. 1984, PASJ, 36, 819

[1396] Wallerstein, G. 1992, Nature, 356, 569

[1397] Walter, F., et al. 1982, ApJ, 253, L67

[1398] Wang, J. C. L. 1997, ApJ, 486, L119

[1399] Warwick, R. S., et al. 1981, MNRAS, 197, 865

[1400] Warwick, R. S., et al. 1988, MNRAS, 232, 551

[1401] Watson, M. G., et al. 1978, ApJ, 221, L69

[1402] Watson, M. G., et al. 1978, MNRAS, 183, 35p

[1403] Watson, M. G., et al. 1981, ApJ, 250, 142

[1404] Watson, M. G., et al. 1985, Sp. Sci. Rev., 40, 195

[1405] Webbink, R. F. 1985, Proc. IAU Symp., 113, 541

[1406] Wenzel, W. 1985, IAU Circ., 4059

[1407] Whelan, J. A. J., et al. 1976, MNRAS, 180, 657

[1408] Whelan, J. A. J., et al. 1977, MNRAS, 181, 259

[1409] White, N. E., \& Holt, S. S. 1982, ApJ, 257, 318

[1410] White, N. E., \& Marshall, F. E. 1983, IAU Circ., 3806

[1411] White, N. E., \& Marshall, F. E. 1984, ApJ, 281, 354

[1412] White, N. E., \& Swank, J. H. 1982, ApJ, 253, L61

[1413] White, N. E., \& van Paradijs, J. 1996, ApJ, 473, L29

[1414] White, N. E., et al. 1978, ApJ, 220, 600 
[1415] White, N. E., et al. 1981, ApJ, 247, 994

[1416] White, N. E., et al. 1984, ApJ, 270, 711

[1417] White, N. E., et al. 1984, ApJ, 283, L9

[1418] White, N. E., et al. 1985, ApJ, 296, 475

[1419] White, N. E., et al. 1987, MNRAS, 226, 645

[1420] White, N. E., et al. 1988, ApJ, 324, 363

[1421] White, N. E., et al. 1989, Proc. 23rd ESLAB Symp. (ESA SP-296)

[1422] White, N. E., et al. 1998, IAU Circ., 6927

[1423] Whitlock, L., et al. 1990, A\&A, 238, 140

[1424] Wijnands, R., \& van der Klis, M. 1997, ApJ, 482, L65

[1425] Wijnands, R., \& van der Klis, M. 1998, Nature, 394, 344

[1426] Wijnands, R., \& van der Klis, M. 1998, ApJ, 507, L63

[1427] Wijnands, R., \& van der Klis, M. 1999, A\&A, 345, L35

[1428] Wijnands, R., \& van der Klis, M. 1999, ApJ, 522, 965

[1429] Wijnands, R., \& van der Klis, M. 2000, ApJ, 528, L93

[1430] Wijnands, R., et al. 1996, ApJ, 469, L5

[1431] Wijnands, R., et al. 1996, ApJ, 473, L45

[1432] Wijnands, R., et al. 1997, A\&A, 323, 399

[1433] Wijnands, R.A.D., et al. 1997, ApJ, 479, L141

[1434] Wijnands, R., et al. 1997, ApJ, 490, L157

[1435] Wijnands, R., et al. 1998, ApJ, 493, L87

[1436] Wijnands, R., et al. 1998, ApJ, 496, L39

[1437] Wijnands, R., et al. 1998, ApJ, 504, L35

[1438] Wijnands, R., et al. 1999, ApJ, 512, L39

[1439] Wijnands, R., et al. 1999, ApJ, 526, L33

[1440] Wijnands, R., et al. 2000, IAU Circ., 7369

[1441] Willis, A. J., et al. 1980, ApJ, 237, 596

[1442] Wilms, J., et al. 1999, ApJ, 522, 460

[1443] Wilson, C. A., et al. 1999, ApJ, 513, 464
[1444] Wilson, C. K., \& Rothschild, R. E. 1983, ApJ, 274, 717

[1445] Winkler, C., \& Trams, N. 1998, A\&A, 337, 729

[1446] Wood, A., et al. 1999, IAU Circ., 7274

[1447] Wood, K. S., et al. 1978, IAU Circ., 3203

[1448] Wood, K. S., et al. 1984, ApJS, 56, 507

[1449] Wood, K. S., et al. 1991, ApJ, 379, 295

[1450] Woods, P. M., et al. 1999, ApJ, 517, 431

[1451] Wren, J., \& McKay, T. 2000, IAU Circ., 7394

[1452] Wu, C. C., et al. 1983, PASP, 95, 391

[1453] Yadav, J. S., et al. 1999, ApJ, 517, 935

[1454] Yamaoka, K., et al. 2000, IAU Circ., 7427

[1455] Yamauchi, S., \& Koyama, K. 1990, PASJ, 42, L83

[1456] Yaqoob, T., et al. 1993, MNRAS, 264, 411

[1457] Yu, W., et al. 1997, ApJ, 490, L153

[1458] Zhang, S. N. 1994, IAU Circ., 6046

[1459] Zhang, S. N. 1995, IAU Circ., 6209

[1460] Zhang, S. N., et al. 1994, IAU Circ., 6096

[1461] Zhang, S. N., et al. 1996, A\&AS, 120, 279

[1462] Zhang, S. N., et al. 1997, ApJ, 479, 381

[1463] Zhang, S. N., et al. 1997, ApJ, 482, L155

[1464] Zhang, S. N., et al. 1998, ApJ, 494, L71

[1465] Zhang, W., et al. 1996, ApJ, 469, L29

[1466] Zhang, W., et al. 1997, IAU Circ., 6541

[1467] Zhang, W., et al. 1998, ApJ, 495, L9

[1468] Zhang, W., et al. 1998, ApJ, 500, L167

[1469] Zhang, W., et al. 1998, ApJ, 500, L171

[1470] Zdziarski, A. A., et al. 1998, MNRAS, 301, 435

[1471] Zwarthoed, G. A. A., et al. 1993, A\&A, 267, 101

[1472] Zyeki, P. T., et al. 1997, MNRAS, 309, 561 